\title{
AVERAGING METHOD FOR DIFFERENTIAL EQUATIONS PERTURBED BY DYNAMICAL SYSTEMS
}

\author{
FRANÇOISE PÈNE ${ }^{1}$
}

\begin{abstract}
In this paper, we are interested in the asymptotical behavior of the error between the solution of a differential equation perturbed by a flow (or by a transformation) and the solution of the associated averaged differential equation. The main part of this redaction is devoted to the ascertainment of results of convergence in distribution analogous to those obtained in [10] and [11]. As in [11], we shall use a representation by a suspension flow over a dynamical system. Here, we make an assumption of multiple decorrelation in terms of this dynamical system. We show how this property can be verified for ergodic algebraic toral automorphisms and point out the fact that, for two-dimensional dispersive billiards, it is a consequence of the method developed in [18]. Moreover, the singular case of a degenerated limit distribution is also considered.
\end{abstract}

Mathematics Subject Classification. 34C29, 58J37, 37D50,60F17.

Received September 5, 2001.

\section{CONTENTS}

1. Introduction $\quad 34$

1.1. Description of the problem 34

1.2. Some definitions and notations $\quad 35$

2. Averaging method for a transformation: General results 35

2.1. Hypotheses and main result $\quad 35$

2.2. Asymptotical behavior of covariances 37

2.3. Convergence in distribution of $\left(\boldsymbol{v}_{\boldsymbol{t}}^{\boldsymbol{\varepsilon}}\right)_{\boldsymbol{t}} \quad 38$

2.4. Convergence in distribution of $\left(e_{t}^{\varepsilon}\right)_{t}$ (proof of Th. 2.1.3) 50

3. Averaging method for a suspension flow: General results 54

3.1. Hypothesis and main result $\quad 54$

3.2. Discretisation of the model $\quad 55$

3.3. Estimations in norm 56

3.4. Convergence in distribution $\quad 59$

4. Examples $\quad 63$

4.1. Ergodic toral automorphisms $\quad 63$

4.2. Sinai dispersive billiard with finite horizon 67

5. Optimality of the estimations - Degenerated case 70

Keywords and phrases: Dynamical system, hyperbolicity, billiard, suspension flow, limit theorem, averaging method, perturbation, differential equation.

${ }^{1}$ UBO, Département de Mathématiques, 29285 Brest Cedex, France; e-mail: Francoise.Pene@univ-brest.fr 
5.1. A general result for a dynamical system $\quad 70$

5.2. A general result for a suspension flow $\quad 72$

5.3. Examples

Appendix A. Construction of Young's tower: Recalls 78

Stable and unstable curves $\quad 78$

An extension of $(\boldsymbol{M}, \boldsymbol{\nu}, \boldsymbol{T})$
A partition

A partition $\quad 80$

An extension of $\left(\boldsymbol{M}, \boldsymbol{\nu}, \boldsymbol{T}^{\boldsymbol{d}}\right) \quad 81$

A factor with a quasicompact transfer operator 81

Appendix B. Proof of the strong decorrelation property for billiard transformation 82

An exponential rate of decorrelation for $\left(\boldsymbol{M}, \boldsymbol{\nu}, \boldsymbol{T}^{\boldsymbol{d}}\right) \quad 82$

End of the proof of proposition 4.2.1 85

Appendix C. Sketch of the proof of the martingale approximations 85

Reversed martingale $\quad 86$

$\begin{array}{ll}\text { Direct martingale } & 87\end{array}$

$\begin{array}{lr}\text { References } & 87\end{array}$

\section{INTRODUCTION}

\subsection{Description of the problem}

Let $(\mathcal{M}, \mu)$ be a probability space endowed with a measurable flow $\left(Y_{t}\right)_{t \geq 0}$ preserving $\mu$. Let $f: \mathbf{R}^{d} \times \mathcal{M} \rightarrow \mathbf{R}^{d}$ be a measurable function, uniformly bounded and uniformly Lipschitz continuous in the first parameter. The space $\mathcal{M}$ may be a compact set, $\mu$ a Borel measure, $\left(Y_{t}\right)_{t}$ a continuous flow $\left(i . e .(t, y) \mapsto Y_{t}(y)\right.$ is continuous) and $f$ a continuous function. Actually, we shall make weaker assumptions. We are interested in the study of the behavior of the stochastic processes $\left(X_{t}^{\varepsilon}(x, \cdot)\right)_{t \geq 0}$ solution of the following differential equation (perturbed by $\left.\left(Y_{t}\right)_{t}\right)$ :

$$
\frac{\mathrm{d} X_{t}^{\varepsilon}}{\mathrm{d} t}(x, y)=f\left(X_{t}^{\varepsilon}(x, y), Y_{\frac{t}{\varepsilon}}(y)\right),
$$

with the initial condition $X_{0}^{\varepsilon}(x, y)=x$. Here, $\varepsilon>0$ is a small parameter. We can see the first variable as a slow variable and the second as a fast one. We approximate the process $\left(X_{t}^{\varepsilon}(x, \cdot)\right)_{t}$ by the solution $\left(W_{t}(x)\right)_{t}$ of the associated averaged differential equation:

$$
\frac{\mathrm{d} W_{t}}{\mathrm{~d} t}(x)=\bar{f}\left(W_{t}(x)\right)=\int_{\mathcal{M}} f\left(W_{t}(x), y^{\prime}\right) \mathrm{d} \mu\left(y^{\prime}\right)
$$

with the initial condition $W_{0}(x)=x$. Our goal is to establish results of convergence in distribution for the family of stochastic processes $\left(\left(E_{t}^{\varepsilon}(x, \cdot):=X_{t}^{\varepsilon}(x, \cdot)-W_{t}(x)\right)_{t}\right)_{\varepsilon>0}$ when $\varepsilon$ goes to 0 . Such results have been obtained by Khas'minskii [10] under an assumption of mixing of processes. As Kifer in [11], we use a representation of the system $\left(\mathcal{M}, \mu,\left(Y_{t}\right)_{t}\right)$ by a suspension flow defined over a dynamical probabilised system $(\Omega, \nu, \sigma)$ by a bounded measurable function $\tau: \Omega \rightarrow] 0 ;+\infty\left[\right.$. This leads us to the study of processes $\left(e_{t}^{\varepsilon}(x, \cdot)=x_{t}^{\varepsilon}(x, \cdot)-w_{t}(x)\right)_{t}$, where $\left(x_{t}^{\varepsilon}(x, \omega)\right)_{t}$ (continuous, piecewise $\left.C^{1}\right)$ and $\left(w_{t}(x)\right)_{t}$ are the solutions of the following differential equations:

$$
\frac{\mathrm{d} x_{t}^{\varepsilon}}{\mathrm{d} t}(x, \omega)=F\left(x_{t}^{\varepsilon}(x, \omega), \sigma^{\left.\frac{t}{\varepsilon}\right\rfloor}(\omega)\right) \text { and } \frac{\mathrm{d} w_{t}}{\mathrm{~d} t}(x)=\bar{F}\left(w_{t}(x)\right)=\int_{\Omega} F\left(w_{t}(x), \omega^{\prime}\right) \mathrm{d} \nu\left(\omega^{\prime}\right)
$$

(the first differential equation of (1.1.3) holding only for $t \in \mathbf{R} \backslash \varepsilon \mathbf{Z})$ with the initial conditions $x_{0}^{\varepsilon}(x, \omega)=w_{0}(x)=$ $x$, where $F: \mathbf{R}^{d} \times \Omega \rightarrow \mathbf{R}^{d}$ is a measurable function, uniformly bounded and uniformly Lipschitz continuous in the first parameter. In [11], Kifer proves the convergence in distribution of $\left(\frac{e_{t}^{\varepsilon}(x, \cdot)}{\sqrt{\varepsilon}}\right)_{0 \leq t \leq T_{0}}$ and $\left(\frac{E_{t}^{\varepsilon}(x, \cdot)}{\sqrt{\varepsilon}}\right)_{0 \leq t \leq T_{0}}$ 
(for any real number $T_{0}>0$ ) under an hypothesis of $\alpha$-mixing of filtrations for the system $(\Omega, \nu, \sigma)$. This method cannot be applied to quasihyperbolic algebraic toral automorphisms (at least for filtrations generated by the most classical method). The main purpose of our paper is to prove that Kifer's results are still true under an hypothesis of multiple decorrelation with exponential rate.

First, we shall establish general results for a transformation (Sect. 2) and for a suspension flow (Sect. 3). In Section 4, we give examples to which the method developed in the two previous sections can be applied (algebraic toral automorphisms, billiard). Moreover, we discuss about Kifer's method and our method, in a comparative way. Finally, in Section 5, we give estimations for $\sup _{0 \leq t \leq T_{0}}\left\|e_{t}^{\varepsilon}(x, \cdot)\right\|_{1}$ and for $\sup _{0 \leq t \leq T_{0}}\left\|E_{t}^{\varepsilon}(x, \cdot)\right\|_{1}$ when the limit distribution is degenerated. In this study, we use results of regularity for solutions of a coboundary equation.

This paper (except Sect. 5.3.2) is part of a thesis [14]. Some details of proofs have been removed. Our main example, the billiard flow, has been studied in detail in the first part of [14] (see $[5-7,17])$.

\subsection{Some definitions and notations}

Let $E$ be a set, $g: \mathbf{R}^{d} \times E \rightarrow \mathbf{R}^{d}$ be a function and $k \geq 1$ be an integer. We denote by $D_{1}{ }^{k} g$ the $k^{\text {th }}$ differential in the first variable when it is defined.

If $E$ is a topological space, $g$ is said to be $C_{b}^{k, 0}$ if $g$ is continuous, uniformly bounded, $C^{k}$ in the first variable and if its differentials $D_{1} g, D_{1}^{2} g, \ldots, D_{1}{ }^{k} g$ are continuous and uniformly bounded.

If $E$ is a measurable space, $g$ is said to be $C_{b}^{k, *}$ if $g$ is measurable, uniformly bounded, $C^{k}$ in the first variable and if $D_{1} g, D_{1}^{2} g, \ldots, D_{1}{ }^{k} g$ are measurable and uniformly bounded.

Let $h: \mathbf{R}^{d} \rightarrow \mathbf{R}^{d}$. We denote by $D h$ the differential of $h$ and $J h$ its Jacobian determinant.

We endow $\mathbf{R}^{d}$ with the norm $|\cdot|$ of the supremum in the canonical basis. If $g: E \rightarrow \mathbf{R}^{d}$ is a measurable function defined on a probabilised space, we write $\|g\|_{k}:=\||g|\|_{L^{k}}$, for any real $k \in\left[1 ;+\infty\left[\right.\right.$ and $\|g\|_{\infty}:=\sup _{\omega \in E}|g(\omega)|$.

\section{Averaging method for a transformation: General Results}

\subsection{Hypotheses and main result}

Let $(\Omega, \nu, \sigma)$ be a (probabilised) dynamical system $(i . e .(\Omega, \nu)$ is a probabilised space endowed with a transformation $\sigma: \Omega \rightarrow \Omega$ preserving the probability measure $\nu$ ). Let a measurable function $F: \mathbf{R}^{d} \times \Omega \rightarrow \mathbf{R}^{d}$ uniformly bounded and uniformly Lipschitz continuous in the first parameter be given. We shall write $\bar{F}(x):=$ $\int_{\Omega} F(x, \omega) \mathrm{d} \nu(\omega), \tilde{F}(x, \omega):=F(x, \omega)-\bar{F}(x)$ and $\tilde{F}_{i}$ the $i^{\text {th }}$ coordinate of $\tilde{F}$. We are interested in the study of the process $\left(e_{t}^{\varepsilon}(x, \cdot)=x_{t}^{\varepsilon}(x, \cdot)-w_{t}(x)\right)_{t}$ given by $(1.1 .3)$ with the initial conditions $x_{0}^{\varepsilon}(x, \cdot)=w_{0}(x)=x$. This problem may be considered independently of the averaging problem in terms of flow. In particular, we mention the existence of an algorithmic (iterative) version ( $c f$. [11]), the study of which leads us to the study of processes $\left(x_{t}^{\varepsilon}(x, \cdot)\right)$ and of $\left(w_{t}(x)\right)$. Kifer has studied the problem when the dynamical system satisfies an $\alpha$-mixing condition. Here we shall make an assumption of multiple decorrelation. Despite its technical complications, we shall see that the method developed here can be applied to several examples (cf. Sect. 4).

Definition 2.1.1. We say that a normed vector space $(V,\|\cdot\|)$ of measurable and uniformly bounded realvalued functions defined on $\Omega$, such that $\|\cdot\|_{\infty} \leq\|\cdot\|$, containing the constant functions satisfies the multiple decorrelation property if the following condition holds:

For any integers $m \geq 1$ and $m^{\prime} \geq 1$, for any real number $r>1$, there exist two constants $C_{r}=C_{r, m, m^{\prime}}>0$ and $\left.\alpha_{r}=\alpha_{r, m, m^{\prime}} \in\right] 0,1\left[\right.$ such that, for any integer $N \geq 0$, any increasing sequences of positive integers $\left(k_{1}, \ldots, k_{m}\right)$ and $\left(l_{1}, \ldots, l_{m^{\prime}}\right)$ and any functions $G_{1}, \ldots, G_{m}, H_{1}, \ldots, H_{m^{\prime}}$ belonging to $V$, we have

$$
\left|\operatorname{Cov}\left(\prod_{i=1}^{m} G_{i} \circ \sigma^{k_{i}} ; \prod_{j=1}^{m^{\prime}} H_{j} \circ \sigma^{N+l_{j}}\right)\right| \leq C_{r}\left(\prod_{i=1}^{m}\left\|G_{i}\right\|\right)\left(\prod_{j=1}^{m^{\prime}}\left\|H_{j}\right\|\right) \alpha_{r}{ }^{N-r \max \left(k_{m}, l_{m^{\prime}}\right)} .
$$


In the following, the fact that the real number $r>1$ can be taken as close to 1 as we want is very important. We can notice that, in the following, this multiple decorrelation property with exponential $\operatorname{rate}\left(\alpha_{r}{ }^{N-r} \max \left(k_{m}, l_{m^{\prime}}\right)\right)$ may be replaced by a multiple decorrelation property with "sub-exponential" rate $\left(\alpha_{r} \sqrt{N-r \max \left(k_{m}, l_{m^{\prime}}\right)}\right)$ or, even, "over-polynomial" rate $\left(f_{r, m, m^{\prime}}\left(N-r \max \left(k_{m}, l_{m^{\prime}}\right)\right)\right.$ with $\lim _{n \rightarrow+\infty} n^{p} \cdot f_{r, m, m^{\prime}}(n)=0$, for any $p \geq 0$, any $m, m^{\prime} \geq 1$ and any real number $\left.r>1\right)$. Those weaker forms shall not be useful for the examples considered in the following. We can observe that, for our examples, $C_{r, m, m^{\prime}}$ and $\alpha_{r, m, m^{\prime}}$ depend neither on $m$ nor on $m^{\prime}$. Moreover, we notice that, in the following, this multiple decorrelation property can be replaced by a condition of the following kind:

$$
\left|\operatorname{Cov}\left(\prod_{i=1}^{m} G_{i} \circ \sigma^{k_{i}} ; \prod_{j=1}^{m^{\prime}} H_{j} \circ \sigma^{N+l_{j}}\right)\right| \leq C_{r}\left(\prod_{i=1}^{m}\left\|G_{i}\right\|\right)\left(\prod_{j=1}^{m^{\prime}}\left\|H_{j}\right\|\right) \alpha_{r}{ }^{N-r_{0} \cdot k_{m}}
$$

for some $C>0$, some $\alpha \in] 0 ; 1\left[\right.$ and some $r_{0}>1$ (cf. Prop. 4.2.1 for the billiard system).

Hypothesis 2.1.2. The function $F: \mathbf{R}^{d} \times \Omega \rightarrow \mathbf{R}^{d}$ is $C_{b}^{2, *}$ (i.e. measurable, uniformly bounded, $C^{2}$ in the first parameter with differentials $D_{1} F$ and $D_{1}^{2} F$ measurable and uniformly bounded).

Moreover, the set of functions $\tilde{F}_{i}(x, \cdot)$ (with $x \in \mathbf{R}^{d}$ and $i=1, \ldots, d$ ) is contained in a normed vector space $(V,\|\cdot\|)$ satisfying the multiple decorrelation property and $\tilde{F}$ satisfies the following condition:

$$
\|\tilde{F}\|:=\sup _{x \in \mathbf{R}^{d}} \sup _{i=1, \ldots, d}\left\|\tilde{F}_{i}(x, \cdot)\right\|<+\infty .
$$

We fix a real number $T_{0}>0$. The main result of this part is the following:

Theorem 2.1.3. Under Hypothesis 2.1.2, for any integer $L \geq 1$, we have

$$
\sup _{0<\varepsilon<1} \sup _{x \in \mathbf{R}^{d}} \sup _{0 \leq t \leq T_{0}}\left\|\frac{e_{t}^{\varepsilon}(x, \cdot)}{\sqrt{\varepsilon}}\right\|_{L}<+\infty .
$$

Moreover, for any $x \in \mathbf{R}^{d}$, the family of processes $\left(\left(\varepsilon^{-\frac{1}{2}} e_{t}^{\varepsilon}(x, \cdot)\right)_{0 \leq t \leq T_{0}}\right)_{\varepsilon>0}$ converges in distribution (for $\nu$ and for the uniform topology on $\left.C\left(\left[0, T_{0}\right]\right)\right)$, when $\varepsilon$ goes to 0 , to the Gaussian process $\left(e_{t}^{0}(x, \cdot)\right)_{0 \leq t \leq T_{0}}$ solution of

$$
e_{t}^{0}(x, \cdot)=v_{t}(x, \cdot)+\int_{0}^{t} D \bar{F}\left(w_{s}(x)\right) \cdot e_{s}^{0}(x, \cdot) \mathrm{d} s
$$

where $\left(v_{t}(x, \cdot)\right)_{t}$ is a continuous Gaussian process with independent increments, centered and such that:

$$
\operatorname{Cov}\left(v_{t}(x, \cdot)\right)=\left(\int_{0}^{t} a_{i, j}\left(w_{s}(x)\right) \mathrm{d} s\right)_{i, j}
$$

with $a_{i, j}(x):=\lim _{t \rightarrow+\infty} \frac{1}{t} \int_{0}^{t} \int_{0}^{t} \mathbf{E}_{\nu}\left[\tilde{F}_{i}\left(x, \sigma^{\lfloor s\rfloor}(\cdot)\right) \cdot \tilde{F}_{j}\left(x, \sigma^{\lfloor u\rfloor}(\cdot)\right)\right] \mathrm{d} s \mathrm{~d} u$.

We see that $\left(e_{t}^{0}(x, \cdot)\right)_{t}$ is given by $e_{t}^{0}(x, \cdot)=v_{t}(x, \cdot)+\int_{0}^{t} D \bar{F}\left(w_{s}(x)\right) \mathrm{e}_{s}^{t} D \bar{F}\left(w_{u}(x)\right) \mathrm{d} u v_{s}(x, \cdot) \mathrm{d} s$. Thus, by Itô formula, we have $\left.\left(e_{t}^{0}(x, \cdot)\right)_{t \geq 0} \stackrel{\text { a.e. }}{=}\left(\int_{0}^{t} \mathrm{e}^{\int_{s}^{t} D \bar{F}\left(w_{u}(x)\right) \mathrm{d} u} \mathrm{~d} v_{s}(x, \cdot)\right)\right)_{t \geq 0}$. For any $\varepsilon>0$ and any $t \in\left[0 ; T_{0}\right]$, we denote by $v_{t}^{\varepsilon}(x, \cdot)$ the random variable given by:

$$
v_{t}^{\varepsilon}(x, \omega):=\frac{1}{\sqrt{\varepsilon}} \int_{0}^{t} \tilde{F}\left(w_{u}(x), \sigma^{\left\lfloor\frac{u}{\varepsilon}\right\rfloor}(\omega)\right) \mathrm{d} u=\sqrt{\varepsilon} \int_{0}^{\frac{t}{\varepsilon}} \tilde{F}\left(w_{\varepsilon s}(x), \sigma^{\lfloor s\rfloor}(\omega)\right) \mathrm{d} s .
$$


The remainder of this section is devoted to the proof of Theorem 2.1.3. We shall see that the study of $\left(v_{t}^{\varepsilon}(x, \cdot)\right)_{t}$ is useful for our purpose. In the following, we shall suppose that the dynamical system $(\Omega, \nu, \sigma)$ is invertible (let us recall that any dynamical system has an invertible extension).

\subsection{Asymptotical behavior of covariances}

Using Gronwall lemma, we get the following inequality.

Remark 2.2.1. For any $t \in\left[0 ; T_{0}\right]$, any $\varepsilon>0$ and any $(x, \omega) \in \mathbf{R}^{d} \times \Omega$, we have

$$
\frac{1}{\sqrt{\varepsilon}}\left|e_{t}^{\varepsilon}(x, \omega)\right| \leq\left|v_{t}^{\varepsilon}(x, \omega)\right|+L_{F} \cdot \mathrm{e}^{L_{F} T_{0}} \int_{0}^{t}\left|v_{s}^{\varepsilon}(x, \omega)\right| \mathrm{d} s .
$$

From this, we get the first estimation of Theorem 2.1 .3 for $L=2$ by proving that we have

$$
\sup _{0<\varepsilon<1} \sup _{x \in \mathbf{R}^{d}} \sup _{0 \leq t \leq T_{0}}\left\|v_{t}^{\varepsilon}(x, \cdot)\right\|_{L^{2}(\Omega, \nu)}<+\infty
$$

which is a consequence of the fact that the following quantity is finite

$$
\sup _{i, j=1, \ldots, d} \sum_{n=-\infty}^{+\infty} \sup _{x, y \in \mathbf{R}^{d}}\left|\mathbf{E}_{\nu}\left[\tilde{F}_{i}(x, \cdot) \cdot \tilde{F}_{j}\left(y, \sigma^{n}(\cdot)\right)\right]\right| .
$$

Moreover, the two following results, which follow from [10,11], give additional information about the behavior of the family of processes $\left(\left(v_{t}^{\varepsilon}(x, \cdot)\right)_{0 \leq t \leq T_{0}}\right)_{\varepsilon>0}$ when $\varepsilon$ goes to 0 . Let $G: \mathbf{R}^{d} \times \Omega \rightarrow \mathbf{R}^{d}$ be a measurable function, uniformly bounded, uniformly Lipschitz continuous in the first parameter and such that the following quantity is finite:

$$
K_{G}:=\sup _{i, j=1, \ldots, d} \sum_{n=-\infty}^{+\infty}(1+|n|) \cdot \sup _{x, y \in \mathbf{R}^{d}}\left|\mathbf{E}_{\nu}\left[G_{i}(x, \cdot) \cdot G_{j}\left(y, \sigma^{n}(\cdot)\right)\right]\right| .
$$

We then consider the processes $\left(V_{t}^{\varepsilon}(x, \cdot)\right)_{t}$ given by

$$
V_{t}^{\varepsilon}(x, \omega):=\frac{1}{\sqrt{\varepsilon}} \int_{0}^{t} G\left(w_{u}(x), \sigma^{\left\lfloor\frac{u}{\varepsilon}\right\rfloor}(\omega)\right) \mathrm{d} u=\sqrt{\varepsilon} \int_{0}^{\frac{t}{\varepsilon}} G\left(w_{\varepsilon s}(x), \sigma^{\lfloor s\rfloor}(\omega)\right) \mathrm{d} s .
$$

Proposition 2.2.2 (cf. [11]). For any $i, j=1, \ldots, d$ and any $x \in \mathbf{R}^{d}$, the following quantity is well defined

$$
a_{i, j}(x):=\sum_{n=-\infty}^{+\infty} \mathbf{E}_{\nu}\left[G_{i}(x, \cdot) G_{j}\left(x, \sigma^{n}(\cdot)\right)\right]
$$

and, for any real numbers $t_{0}, t>0$ and any $x$ in $\mathbf{R}^{d}$, we have

$$
\left|a_{i, j}(x)-\frac{1}{t} \int_{t_{0}}^{t_{0}+t} \int_{t_{0}}^{t_{0}+t} \mathbf{E}_{\nu}\left[G_{i}\left(x, \sigma^{\lfloor s\rfloor}(\cdot)\right) \cdot G_{j}\left(x, \sigma^{\lfloor u\rfloor}(\cdot)\right)\right] \mathrm{d} s \mathrm{~d} u\right| \leq \frac{2 K_{G}}{t} .
$$

Proposition 2.2.3 (cf. [10]). Let us suppose that $K_{G}$ is finite. Then, for any $i, j=1, \ldots, d$, any $x \in \mathbf{R}^{d}$ and any real numbers $s, t$ satisfying $0<s<t$, we have

$$
\lim _{\varepsilon \rightarrow 0} \mathbf{E}_{\nu}\left[\left(V_{t}^{\varepsilon}(x, \cdot)-V_{s}^{\varepsilon}(x, \cdot)\right)_{i} \cdot\left(V_{t}^{\varepsilon}(x, \cdot)-V_{s}^{\varepsilon}(x, \cdot)\right)_{j}\right]=\int_{s}^{t} a_{i, j}\left(w_{u}(x)\right) \mathrm{d} u .
$$


Sketch of the proof. We first notice that we have

$$
\mathbf{E}_{\nu}\left[\left(V_{t}^{\varepsilon}(x, \cdot)-V_{s}^{\varepsilon}(x, \cdot)\right)_{i} \cdot\left(V_{t}^{\varepsilon}(x, \cdot)-V_{s}^{\varepsilon}(x, \cdot)\right)_{j}\right]=\varepsilon \int_{\frac{s}{\varepsilon}}^{\frac{t}{\varepsilon}} \int_{\frac{s}{\varepsilon}}^{\frac{t}{\varepsilon}} \tilde{C}_{i, j}^{\lfloor u\rfloor,\lfloor v\rfloor}(u, v) \mathrm{d} u \mathrm{~d} v,
$$

with $\tilde{C}_{i, j}^{m, n}(u, v):=\mathbf{E}_{\nu}\left[G_{i}\left(w_{\varepsilon u}(x), \sigma^{m}(\cdot)\right) \cdot G_{j}\left(w_{\varepsilon v}(x), \sigma^{n}(\cdot)\right)\right]$. Let a real number $\left.\gamma \in\right] \frac{1}{2} ; 1[$ be fixed. We denote by $H_{\varepsilon}$ the square $\left[\frac{s}{\varepsilon} ; \frac{t}{\varepsilon}\right]^{2}$ and $n_{\varepsilon}:=\left\lfloor\varepsilon^{-\gamma}\right\rfloor$. For any integer $k=0, \ldots, n_{\varepsilon}$, we write $s_{k, \varepsilon}:=\frac{s}{\varepsilon}+k \frac{t-s}{\varepsilon n_{\varepsilon}}$. We then consider the diagonal set $A_{\varepsilon}:=\bigcup_{k=0}^{n_{\varepsilon}-1}\left[s_{k, \varepsilon}, s_{k+1, \varepsilon}\right]^{2}$. We have:

$$
\varepsilon \iint_{H_{\varepsilon} \backslash A_{\varepsilon}}\left|\tilde{C}_{i, j}^{\lfloor u\rfloor,\lfloor v\rfloor}(u, v)\right| \mathrm{d} u \mathrm{~d} v \leq 2 K_{G} \varepsilon n_{\varepsilon}
$$

and

$$
\varepsilon \sum_{k=0}^{n_{\varepsilon}-1} \int_{s_{k, \varepsilon}}^{s_{k+1, \varepsilon}} \int_{s_{k, \varepsilon}}^{s_{k+1, \varepsilon}}\left|\tilde{C}_{i, j}^{\lfloor u\rfloor,\lfloor v\rfloor}(u, v)-\tilde{C}_{i, j}^{\lfloor u\rfloor,\lfloor v\rfloor}\left(s_{k, \varepsilon}, s_{k, \varepsilon}\right)\right| \mathrm{d} u \mathrm{~d} v \leq \frac{(t-s)^{3}}{\varepsilon n_{\varepsilon}^{2}} 2 L_{G}\|\bar{F}\|_{\infty}\|G\|_{\infty}
$$

Moreover, we have

$$
\left|\varepsilon \iint_{A_{\varepsilon}} \tilde{C}_{i, j}^{\lfloor u\rfloor,\lfloor v\rfloor}\left(s_{k, \varepsilon}, s_{k, \varepsilon}\right) \mathrm{d} u \mathrm{~d} v-\frac{t-s}{n_{\varepsilon}} \sum_{k=0}^{n_{\varepsilon}-1} a_{i, j}\left(w_{\varepsilon s_{k, \varepsilon}}(x)\right)\right| \leq 2 K_{G} \varepsilon n_{\varepsilon} .
$$

It remains to show the convergence in distribution (for $\nu$ and for the uniform topology on $\left.C\left(\left[0, T_{0}\right]\right)\right)$ of $\left(\left(\frac{e_{t}^{\varepsilon}(x, \cdot)}{\sqrt{\varepsilon}}\right)_{0 \leq t \leq T_{0}}\right)_{\varepsilon>0}$ to a Gaussian process (when $\varepsilon$ goes to 0 ). First, we study the asymptotical behavior in distribution of $\left(\left(v_{t}^{\varepsilon}(x, \cdot)\right)_{0 \leq t \leq T_{0}}\right)_{\varepsilon>0}$.

\subsection{Convergence in distribution of $(v)$}

We consider a function $G$ satisfying the following assumption:

Hypothesis 2.3.1. The function $G: \mathbf{R}^{d} \times \Omega \rightarrow \mathbf{R}^{d}$ is measurable, uniformly bounded, uniformly $L_{G}$-Lipschitz continuous in the first variable. Moreover, the functions $G_{i}(x, \cdot)\left(\right.$ for $x$ in $\mathbf{R}^{d}$ and $\left.i=1, \ldots, d\right)$ are $\nu$-centered, are contained in a normed vector space $(V,\|\cdot\|)$ satisfying the multiple decorrelation property, and $G$ satisfies the following condition:

$$
\|G\|:=\sup _{x \in \mathbf{R}^{d}} \sup _{i=1, \ldots, d}\left\|G_{i}(x, \cdot)\right\|<+\infty
$$

We consider again the processes $\left(V_{t}^{\varepsilon}(x, \cdot)\right)_{t}$ defined by $V_{t}^{\varepsilon}(x, \omega)=\frac{1}{\sqrt{\varepsilon}} \int_{0}^{t} G\left(w_{u}(x), \sigma^{\left\lfloor\frac{u}{\varepsilon}\right\rfloor}(\omega)\right) \mathrm{d} u$.

Theorem 2.3.2. Under Hypothesis 2.3.1, for any $x$ in $\mathbf{R}^{d}$, the family of processes $\left(\left(V_{t}^{\varepsilon}(x, \cdot)\right)_{0 \leq t \leq T_{0}}\right)_{\varepsilon>0}$ converges in distribution (for $\nu$ and for the uniform topology on $C\left(\left[0, T_{0}\right]\right)$ ), when $\varepsilon$ goes to 0 , to a continuous Gaussian process $\left(V_{t}(x, \cdot)\right)_{0 \leq t \leq T_{0}}$ centered, with independent increments and such that:

$$
\operatorname{Cov}\left(V_{t}(x, \cdot)\right)=\left(\int_{0}^{t} a_{i, j}\left(w_{s}(x)\right) \mathrm{d} s\right)_{i, j}
$$

with $a_{i, j}(x):=\lim _{t \rightarrow+\infty} \frac{1}{t} \int_{0}^{t} \int_{0}^{t} \mathbf{E}_{\nu}\left[G_{i}\left(x, \sigma^{\lfloor s\rfloor}(\cdot)\right) \cdot G_{j}\left(x, \sigma^{\lfloor u\rfloor}(\cdot)\right)\right] \mathrm{d} s \mathrm{~d} u$. 
To show this theorem, we shall first establish the property of tightness for each family of processes $\left(\left(V_{t}^{\varepsilon}(x, \cdot)\right)_{0 \leq t \leq T_{0}}\right)_{\varepsilon>0}$, then we shall identify the cluster values (for the convergence in distribution for $\nu$ and for the uniform topology on $\left.C\left(\left[0, T_{0}\right]\right)\right)$ by proving a result of convergence in the sense of the finite distributions.

\section{A result of tightness}

Proposition 2.3.3. Under Hypothesis 2.3.1, for any $x$ in $\mathbf{R}^{d}$, the family of processes $\left(\left(V_{t}^{\varepsilon}(x, \cdot)\right)_{0 \leq t \leq T_{0}}\right)_{\varepsilon>0}$ is tight.

Proof. According to Kolmogorov's criterion (cf. [16], for example), it is enough to show that we have:

$$
\sup _{0<\varepsilon<1} \sup _{0 \leq t<t+h \leq T_{0}} \frac{1}{h^{2}}\left\|V_{t+h}^{\varepsilon}(x, \cdot)-V_{t}^{\varepsilon}(x, \cdot)\right\|_{4}^{4}<+\infty .
$$

We write $N_{\varepsilon, t, h}:=\left\lceil\frac{t+h}{\varepsilon}\right\rceil-\left\lfloor\frac{t}{\varepsilon}\right\rfloor$. The quantity $\frac{1}{h^{2}}\left\|V_{t+h}^{\varepsilon}(x, \cdot)-V_{t}^{\varepsilon}(x, \cdot)\right\|_{4}^{4}$ is less than the following quantity:

$$
\frac{\varepsilon^{2}}{h^{2}} \sum_{n_{1}, n_{2}, n_{3}, n_{4}=0}^{N_{\varepsilon, t, h}-1} \int_{n_{1}}^{n_{1}+1} \int_{n_{2}}^{n_{2}+1} \int_{n_{3}}^{n_{3}+1} \int_{n_{4}}^{n_{4}+1}\left|\mathbf{E}_{\nu}\left[\prod_{j=1}^{4} G_{i}\left(w_{\varepsilon\left(\left\lfloor\frac{t}{\varepsilon}\right\rfloor+u_{j}\right)}(x), \sigma^{n_{j}}(\cdot)\right)\right]\right| \mathrm{d} u
$$

with $\mathrm{d} u=\mathrm{d} u_{1} \mathrm{~d} u_{2} \mathrm{~d} u_{3} \mathrm{~d} u_{4}$. We conclude by the following lemma.

Lemma 2.3.4. Let $(V,\|\cdot\|)$ be a normed vector space satisfying the multiple decorrelation property. Let an integer $L \geq 1$ and two real numbers $M_{\infty}>0$ and $M>0$ be given. We denote by $\mathcal{E}_{L, M_{\infty}, M}$ the set of functions $H=\left(H^{(1)}, \ldots, H^{(L)}\right) \in V^{L}$ satisfying:

$$
\forall i=1, \ldots, L, \quad\left\|H^{(i)}\right\|_{\infty} \leq M_{\infty},\left\|H^{(i)}\right\| \leq M, \mathbf{E}_{\nu}\left[H^{(i)}\right]=0
$$

We have

$$
\sup _{N \geq 1} \frac{1}{N^{\frac{L}{2}}} \sum_{n_{1}, \ldots, n_{L}=0}^{N-1} \sup _{H \in \mathcal{E}_{L, M_{\infty}, M}}\left|H_{n_{1}, \ldots, n_{L}}^{(1, \ldots, L)}\right|<+\infty, \quad \text { with } H_{n_{1}, \ldots, n_{L}}^{\left(i_{1}, \ldots, i_{L}\right)}:=\mathbf{E}_{\nu}\left[\prod_{k=1}^{L} H^{\left(i_{k}\right)} \circ \sigma^{n_{k}}\right] .
$$

Proof. This result is based on the multiple decorrelation property and on a classical combinatory argument. We define

$$
B_{L, N}:=\sum_{0 \leq l_{1}, \ldots, l_{L} \leq N-1} \sup _{H \in \mathcal{E}_{L, M_{\infty}, M}}\left|H_{l_{1}, \ldots, l_{L}}^{(1, \ldots, L)}\right|
$$

for any integers $L \geq 1$ and $N \geq 1$. We shall show, inductively on $L \geq 1$, that we have $\sup _{N \geq 1} N^{-\frac{p}{2}} B_{p, N}<+\infty$ for any integer $p=1, \ldots, L$. We first notice that this is true for $L=2$. Indeed, we have $\sup _{N \geq 1} B_{1, N}=0$ and $\sup _{N \geq 1} N^{-1} B_{2, N} \leq \sup _{N \geq 1} \frac{1}{N} \sum_{k, l=0}^{N-1} C_{r, 1,1} M^{2}\left(\alpha_{r, 1,1}\right)^{|k-l|}<+\infty$.

Let us suppose that the result is true for an integer $L \geq 2$ and show that then it is also true for $L+1$. Let $N \geq 1$ be an integer. For all finite sequence $\mathbf{l}=\left(l_{1}, \ldots, l_{L+1}\right) \in\{0, \ldots, N-1\}^{L+1}$, we denote by $\alpha(i)=\alpha(i)(\mathbf{l})$ the index of the $i^{\text {th }}$ smallest component of $\mathbf{l}$ :

$$
\begin{aligned}
\alpha(1) & :=\min \left\{j: l_{j} \leq l_{k}, \forall k\right\} \\
\alpha(i+1) & :=\min \left\{j \neq \alpha(1), \ldots, \alpha(i): l_{j} \leq l_{k}, \forall k \neq \alpha(1), \ldots, \alpha(i)\right\}
\end{aligned}
$$

For any $i=1, \ldots, L+1$, we denote by $m_{i}=m_{i}(\mathbf{l})=l_{\alpha(i)}$ the $i^{\text {th }}$ smallest component of $l$. We then have $B_{L+1, N}=\sum_{\mathbf{l}} \sup _{H \in \mathcal{E}_{L+1, M_{\infty}, M}}\left|H_{m_{1}(\mathbf{l}), \ldots, m_{L+1}(\mathbf{l})}^{(\alpha(1), \ldots, \alpha(L+1)}\right|$. We denote, for any $i=0, \ldots, L, k_{i}:=m_{i+1}-m_{1}$. Let $\beta$ be a 
real number in $] 0 ; \frac{L-1}{2 L}[$. We fix a real number $r>1$ (small enough) such that we have:

$$
\frac{r^{L}}{4 r-1} \leq \frac{1}{2} \text { and } 1+r-r^{L-j+1}-\frac{r^{L-j+1}}{4 r-1} \geq \frac{1}{2}
$$

for any $j=1, \ldots, L-1$. We write $C_{r}:=\sup _{m+m^{\prime}=L+1} C_{r, m, m^{\prime}}$. We define the following sets:

$$
\begin{aligned}
& E^{(L+1)}:=\left\{\mathbf{l}=\left(l_{1}, \ldots, l_{L+1}\right) \in \mathbf{N}^{L}: 0 \leq l_{1}, \ldots, l_{L+1} \leq N-1\right\} \\
& E_{\beta, 0}^{(L+1)}:=\left\{\mathbf{l}=\left(l_{1}, \ldots, l_{L+1}\right) \in E^{(L+1)}: k_{j}(\mathbf{l})-r k_{j-1}(\mathbf{l}) \leq \frac{N^{\beta}}{4^{j}}, \forall j=1, \ldots, L\right\}, \\
& E_{\beta, L}^{(L+1)}:=\left\{\mathbf{l}=\left(l_{1}, \ldots, l_{L+1}\right) \in E^{(L+1)}: k_{L}(\mathbf{l})-r k_{L-1}(\mathbf{l})>\frac{N^{\beta}}{4^{L}}\right\}, \\
& E_{\beta, j}^{(L+1)}:=\left\{\mathbf{l}=\left(l_{1}, \ldots, l_{L+1}\right) \in E^{(L+1)} \backslash\left(E_{\beta, j+1}^{(L+1)} \cup \cdots \cup E_{\beta, L}^{(L+1)}\right): k_{j}(\mathbf{l})-r k_{j-1}(\mathbf{l})>\frac{N^{\beta}}{4^{j}}\right\},
\end{aligned}
$$

for any $j=1, \ldots, L-1$. We have $E^{(L+1)}=\bigcup_{j=0}^{L} E_{\beta, j}^{(L+1)}$. For any subset $B \subseteq E^{(L+1)}$, we denote

$$
S(B):=\sum_{\left(l_{1}, \ldots, l_{L+1}\right) \in B} \sup _{H \in \mathcal{E}_{L+1, M \infty}, M}\left|H_{l_{1}, \ldots, l_{L+1}}^{(1, \ldots, L+1)}\right| .
$$

We have $B_{L+1, N}=\sum_{j=0}^{L} S\left(E_{\beta, j}^{(L+1)}\right)$.

1. In a first time, we give a bound for $\# E_{\beta, 0}^{(L+1)}$. Let $\mathbf{l}=\left(l_{1}, \ldots, l_{L+1}\right)$ be in $E_{\beta, 0}^{(L+1)}$. We show, inductively on $i$, that, for any integer $i=1, \ldots, L$, we have $k_{i} \leq \sum_{l=1}^{i} r^{i-l} \frac{N^{\beta}}{4^{l}}$. Indeed, we have $k_{1} \leq \frac{N^{\beta}}{4}$. Let us suppose the result true for $i-1$ (for some $i=2, \ldots, L$ ) and let us show that it is then still true for $i$. We have

$$
k_{i} \leq r k_{i-1}+\frac{N^{\beta}}{4^{i}} \leq r \sum_{l=1}^{i-1} r^{(i-1)-l} \frac{N^{\beta}}{4^{l}}+\frac{N^{\beta}}{4^{i}}=\sum_{l=1}^{i-1} r^{i-l} \frac{N^{\beta}}{4^{l}}+\frac{N^{\beta}}{4^{i}} .
$$

So, we have, for any $i=1, \ldots, L, k_{i}=m_{i+1}-m_{1} \leq \sum_{l=1}^{L} r^{L-l} \frac{N^{\beta}}{4^{l}} \leq N^{\beta} \frac{r^{L}}{4 r-1} \leq \frac{N^{\beta}}{2}$ and

$$
S\left(E_{\beta, 0}^{(L+1)}\right) \leq(L+1) !\left(\frac{3 M_{\infty}}{2}\right)^{L+1} N^{1+L \beta}
$$

Besides, we recall that we have $1+L \beta<\frac{L+1}{2}$.

2. If $\mathbf{l}=\left(l_{1}, \ldots, l_{L+1}\right) \in E_{\beta, L}^{(L+1)}$ and $H \in \mathcal{E}_{L+1, M_{\infty}, M}$, then we have

$$
\begin{aligned}
\left|H_{l_{1}, \ldots, l_{L+1}}^{(1, \ldots, L+1)}\right| & =\left|\operatorname{Cov}\left(\prod_{i=1}^{L} H^{(\alpha(i))} \circ \sigma^{k_{i-1}}, H^{(\alpha(L+1))} \circ \sigma^{k_{L}}\right)\right| \\
& \leq C_{r} M^{L+1} \alpha_{r}{ }^{k_{L}(\mathbf{l})-r k_{L-1}(\mathbf{l})} \\
& \leq C_{r} M^{L+1} \alpha_{r}{ }^{\frac{N^{\beta}}{4^{L}}}
\end{aligned}
$$

according to the multiple decorrelation property. We get $S\left(E_{\beta, L}^{(L+1)}\right) \leq N^{L+1} C_{r} M^{L+1} \alpha_{r}^{\frac{N^{\beta}}{4 L}}$. 
3. Let $j=1, \ldots, L-1$. If $\mathbf{l}=\left(l_{1}, \ldots, l_{L+1}\right) \in E_{\beta, j}^{(L+1)}$ and $H \in \mathcal{E}_{L+1, M_{\infty}, M}$, then we have: $\left|H_{l_{1}, \ldots, l_{L+1}}^{(1, \ldots, L+1)}\right| \leq\left|\operatorname{Cov}\left(\prod_{i=1}^{j} H^{(\alpha(i))} \circ \sigma^{k_{i-1}}, \prod_{i=j+1}^{L+1} H^{(\alpha(i))} \circ \sigma^{k_{i-1}}\right)\right|+\left|H_{m_{1}, \ldots, m_{j}}^{(\alpha(1), \ldots, \alpha(j))}\right| \cdot\left|H_{m_{j+1}, \ldots, m_{L+1}}^{(\alpha(j+1), \ldots, \alpha(L+1))}\right|$

By the multiple decorrelation property, we have

$$
\left|\operatorname{Cov}\left(\prod_{i=1}^{j} H^{(\alpha(i))} \circ \sigma^{k_{i-1}}, \prod_{i=j+1}^{L+1} H^{(\alpha(i))} \circ \sigma^{k_{i-1}}\right)\right| \leq C_{r} M^{L+1} \alpha_{r}{ }^{k_{j}-r \max \left(k_{j-1}, k_{L}-k_{j}\right)}
$$

We have $k_{j}-r k_{j-1} \geq \frac{N^{\beta}}{4^{j}}$. For any $i=j+1, \ldots, L$, we have $k_{i} \leq r k_{i-1}+\frac{N^{\beta}}{4^{i}}$. We show, by a decreasing induction, that we have, for any $i=j, \ldots, L$,

$$
k_{L} \leq r^{L-i} k_{i}+\sum_{l=1}^{L-i} r^{L-i-l} \frac{N^{\beta}}{4^{i+l}}
$$

Indeed, this is satisfied for $i=L$. Let us suppose that this inequality is satisfied for an integer $i \in$ $\{j+1, \ldots, L\}$ and show that it is then true for $i-1$. Then, we have

$$
\begin{aligned}
k_{L} & \leq r^{L-i} k_{i}+\sum_{l=1}^{L-i} r^{L-i-l} \frac{N^{\beta}}{4^{i+l}} \\
& \leq r^{L-i}\left(r k_{i-1}+\frac{N^{\beta}}{4^{i}}\right)+\sum_{l=1}^{L-i} r^{L-i-l} \frac{N^{\beta}}{4^{i+l}} \\
& \leq r^{L-(i-1)} k_{i-1}+r^{L-(i-1)-1} \frac{N^{\beta}}{4^{(i-1)+1}}+\sum_{l=1}^{L-i} r^{L-(i-1)-(l+1)} \frac{N^{\beta}}{4^{(i-1)+(l+1)}} \\
& \leq r^{L-(i-1)} k_{i-1}+\sum_{l=1}^{L-(i-1)} r^{L-(i-1)-l} \frac{N^{\beta}}{4^{(i-1)+l}} .
\end{aligned}
$$

Using this inequality for $i=j$, we get:

$$
\begin{aligned}
k_{j}-r\left(k_{L}-k_{j}\right) & \geq k_{j}-r\left(r^{L-j} k_{j}+\sum_{l=1}^{L-j} r^{L-j-l} \frac{N^{\beta}}{4^{j+l}}-k_{j}\right) \\
& \geq k_{j}\left(1+r-r^{L-j+1}\right)-\sum_{l=1}^{L-j} r^{L-j-l+1} \frac{N^{\beta}}{4^{j+l}} \\
& \geq \frac{N^{\beta}}{4^{j}}\left(1+r-r^{L-j+1}-r^{L-j+1} \sum_{l=1}^{L-j} \frac{r^{-l}}{4^{l}}\right) \\
& \geq \frac{N^{\beta}}{4^{j}}\left(1+r-r^{L-j+1}-\frac{r^{L-j+1}}{4 r-1}\right) \\
& \geq \frac{N^{\beta}}{2.4^{j}} \geq \frac{N^{\beta}}{4^{L}} .
\end{aligned}
$$


We recall that we have $B_{L+1, N}=\sum_{j=0}^{L+1} S\left(E_{\beta, j}^{(L+1)}\right)$. Furthermore, we have

$$
S\left(E_{\beta, 0}^{(L+1)}\right)+S\left(E_{\beta, L}^{(L+1)}\right) \leq N^{\frac{L+1}{2}}\left((L+1) ! N^{1+L \beta-\frac{L+1}{2}}\left(\frac{3 M_{\infty}}{2}\right)^{L+1}+C_{r} M^{L+1} N^{\frac{L+1}{2}} \alpha_{r}^{\frac{N^{\beta}}{4}}\right)
$$

and, for any $j=1, \ldots, L-1$,

$$
\begin{aligned}
\frac{1}{N^{\frac{L+1}{2}}} S\left(E_{\beta, j}^{(L+1)}\right) & \leq C_{r} M^{L+1} N^{\frac{L+1}{2}} \alpha_{r} \frac{N^{\beta}}{4 L}+\frac{1}{N^{\frac{L+1}{2}}} \sum_{\mathrm{l} \in E^{(L+1)}} \sup _{H \in \mathcal{E}_{L+1, M \infty, M}}\left|H_{m_{1}, m_{2}, \ldots, m_{j}}^{(\alpha(1), \ldots, \alpha(j))}\right| \cdot\left|H_{m_{j+1}, \ldots, m_{L+1}}^{(\alpha(j+1), \ldots, \alpha(L+1))}\right| \\
& \leq C_{r} M^{L+1} N^{\frac{L+1}{2}} \alpha_{r} \frac{N^{\alpha}}{4^{L}}+(L+1) ! \frac{S\left(E^{(j)}\right)}{N^{\frac{j}{2}}} \frac{S\left(E^{(L+1-j)}\right)}{N^{\frac{L+1-j}{2}}}
\end{aligned}
$$

We notice that the proof of this result is based on the fact that the real number $r>1$, appearing in the multiple decorrelation property, can be taken as close to 1 as we wish.

\section{Asymptotical behavior of finite distributions}

Now, we identify the cluster values of $\left(\left(\left(V_{t}^{\varepsilon}(x, \cdot)\right)_{0 \leq t \leq T_{0}}\right)\right)_{\varepsilon>0}$ for the convergence in distribution (for $\nu$ and for the uniform topology on $\left.C\left(\left[0, T_{0}\right]\right)\right)$. We start with the proof of the following result.

Proposition 2.3.5. Let $x$ be a point in $\mathbf{R}^{d}$ and two real numbers $s, t$ such that $0<s<t$. The family of random variables $\left(V_{t}^{\varepsilon}(x, \cdot)-V_{s}^{\varepsilon}(x, \cdot)\right)_{\varepsilon>0}$ converges in distribution for $\nu$ (when $\varepsilon$ goes to 0 ) to a Gaussian random variable $N$ centered, the covariance matrix of which is given by $\left(\int_{s}^{t} a_{i, j}\left(w_{u}(x)\right) \mathrm{d} u\right)_{i, j}$.

The idea of the proof of this proposition is based on the following lemmas:

Lemma 2.3.6 (Method of the moments). Let $\left(X_{n}\right)_{n}$ be a sequence of real random variables such that, for any integer $p \geq 1$, we have $\lim _{n \rightarrow+\infty} \mathbf{E}\left[\left(X_{n}\right)^{p}\right]=\mathbf{E}\left[N^{p}\right]$, where $N$ is a Gaussian random variable of distribution $\mathcal{N}(0, c)$ with $c \geq 0$. Then, $\left(X_{n}\right)_{n}$ converges in distribution to $N$.

Let $\beta$ be a vector in $\mathbf{R}^{d}$. We shall show that, for any integer $L \geq 1$, the moment of order $L$ of $\left(\left\langle\beta, V_{t}^{\varepsilon}(x, \cdot)-V_{s}^{\varepsilon}(x, \cdot)\right\rangle\right)_{\varepsilon>0}$ converges (as $\varepsilon$ goes to 0 ) to the moment of order $L$ of $\langle\beta, N\rangle$, where $N$ is as in the statement of the proposition. According to Lemma 2.3.4, we first observe that, for any integer $L \geq 1$, we have

$$
\sup _{0<\varepsilon<t-s} \sup _{x \in \mathbf{R}^{d}}\left\|\frac{1}{\sqrt{\left\lfloor\frac{t-s}{\varepsilon}\right\rfloor}} \sum_{k=0}^{\left\lfloor\frac{t-s}{\varepsilon}\right\rfloor-1} \int_{\left\lfloor\frac{s}{\varepsilon}\right\rfloor+k}^{\left\lfloor\frac{s}{\varepsilon}\right\rfloor+k+1}\left\langle\beta, G\left(w_{\varepsilon u}(x), \sigma^{k}(\omega)\right)\right\rangle \mathrm{d} u\right\|_{L}<+\infty
$$

and therefore

$$
\sup _{0<\varepsilon<1} \sup _{x \in \mathbf{R}^{d}} \sup _{0 \leq s<t \leq T_{0}}\left\|\frac{V_{t}^{\varepsilon}(x, \cdot)-V_{s}^{\varepsilon}(x, \cdot)}{\sqrt{t-s}}\right\|_{L}<+\infty .
$$

A consequence of this is the first result of Theorem 2.1.3:

$$
\sup _{0<\varepsilon<1} \sup _{x \in \mathbf{R}^{d}} \sup _{0 \leq t \leq T_{0}}\left\|\frac{e_{t}^{\varepsilon}(x, \cdot)}{\sqrt{\varepsilon}}\right\|_{L}<+\infty .
$$

In the same way, the following lemma shall be useful in the proof of the convergence in distribution. 
Lemma 2.3.7. Under hypothesis of Lemma 2.3.4, we have, for any odd integer $p \geq 1$,

$$
\lim _{N \rightarrow+\infty} \frac{1}{N^{\frac{p}{2}}} \sum_{n_{1}, \ldots, n_{p}=0}^{N-1} \sup _{H \in \mathcal{E}_{p, M_{\infty}, M}}\left|H_{n_{1}, \ldots, n_{p}}^{(1, \ldots, p)}\right|=0
$$

and, for any even integer $p \geq 2$,

$$
\lim _{N \rightarrow+\infty} \frac{1}{N^{\frac{p}{2}}} \sum_{l_{1}, \ldots, l_{p}=0}^{N-1} \sup _{H \in \mathcal{E}_{p, M_{\infty}, M}}\left|H_{m_{1}(\mathbf{l}), \ldots, m_{p}(\mathbf{l})}^{(\alpha(1), \ldots,(p))}-\prod_{i=1}^{\frac{p}{2}} H_{m_{2 i-1}(\mathbf{l}), m_{2 i}(\mathbf{l})}^{(\alpha(2 i-1), \alpha(2 i)}\right|=0
$$

where we denote by $\alpha(i)=\alpha(i)(\mathbf{l})$ the index of the $i^{\text {th }}$ smallest component of the integer vector $\mathbf{l}=\left(l_{1}, \ldots, l_{p}\right)$ :

$$
\begin{gathered}
\alpha(1)(\mathbf{l}):=\min \left\{j=1, \ldots, p: l_{j} \leq l_{k}, \forall k\right\} \\
\alpha(i+1)(\mathbf{l}):=\min \left\{j \neq \alpha(1), \ldots, \alpha(i): l_{j} \leq l_{k}, \forall k \neq \alpha(1), \ldots, \alpha(i)\right\}
\end{gathered}
$$

and, for any $i=1, \ldots, p, m_{i}=m_{i}(\mathbf{l}):=l_{\alpha(i)}$.

Proof. We show this result inductively on $p$. It is true for $p=1$ and $p=2$. Let us suppose it true for any $p=1, \ldots, L$, for some integer $L \geq 2$ and let us show that it is then true for $L+1$. Let $r>1$ be a real number as in the proof of the previous lemma. We use the notations introduced in the proof of the previous lemma.

If $L+1$ is odd, then, according to the proof of Lemma 2.3.4, we have $\lim _{N \rightarrow+\infty} \frac{1}{N^{\frac{L+1}{2}}} S\left(E_{\beta, 0}^{(L+1)}\right)=0$ and

$$
\frac{S\left(E_{\beta, j}^{(L+1)}\right)}{N^{\frac{L+1}{2}}} \leq C_{r} M^{L+1} N^{\frac{L+1}{2}} \alpha_{r}^{\frac{N^{\beta}}{4^{L}}}+(L+1) ! \frac{S\left(E^{(j)}\right)}{N^{\frac{j}{2}}} \frac{S\left(E^{(L+1-j)}\right)}{N^{\frac{L+1-j}{2}}}
$$

for any integer $j=1, \ldots, L$. If $j$ is even, then $L+1-j$ is odd and then, according to Lemma 2.3.4 and to the induction hypothesis, we have $\sup _{N \geq 1} \frac{S\left(E^{(j)}\right)}{N^{\frac{j}{2}}}<+\infty$ and $\lim _{N \rightarrow+\infty} \frac{S\left(E^{(L+1-j)}\right)}{N^{\frac{L+1-j}{2}}}=0$. In the same way, if $j$ is odd, we have $\lim _{N \rightarrow+\infty} \frac{S\left(E^{(j)}\right)}{N^{\frac{j}{2}}}=0$ and $\sup _{N \geq 1} \frac{S\left(E^{(L+1-j)}\right)}{N^{\frac{L+1-j}{2}}}<+\infty$.

We suppose that $L+1$ is even. For any subset $B \subseteq E^{(L+1)}$, we denote by $S_{0}(B)$ the following quantity

$$
S_{0}(B):=\sum_{\mathbf{l} \in B} \sup _{H \in \mathcal{E}_{L+1, M_{\infty}, M}}\left|H_{m_{1}(\mathbf{l}), \ldots, m_{L+1}(\mathbf{l})}^{(\alpha(1), \ldots, \alpha(L+1))}-\prod_{i=1}^{\frac{L+1}{2}} H_{m_{2 i-1}(\mathbf{l}), m_{2 i}(\mathbf{l})}^{(\alpha(2 i-1)}\right| .
$$

Then, we have $\frac{S_{0}\left(E_{\beta, 0}^{(L+1)}\right)}{N^{\frac{L+1}{2}}} \leq 2(L+1) !\left(\frac{3 M_{\infty}}{2}\right)^{L+1} N^{1+L \beta-\frac{L+1}{2}}$. Let $j=1, \ldots, L$. We have

$$
\lim _{N \rightarrow+\infty} \frac{1}{N^{\frac{L+1}{2}}} \sum_{\mathbf{l} \in E_{\beta, j}^{(L+1)}} \sup _{H \in \mathcal{E}_{L+1, M_{\infty}, M}} C_{H}^{(j)}\left(m_{1}, \ldots, m_{L+1}\right)=0
$$

with

$$
\begin{aligned}
C_{H}^{(j)}\left(m_{1}, \ldots, m_{L+1}\right) & :=\left|H_{m_{1}, \ldots, m_{L+1}}^{(\alpha(1), \ldots(L+1))}-H_{m_{1}, \ldots, m_{j}}^{(\alpha(1), \ldots, \alpha(j))} H_{m_{j+1}, \ldots, m_{L+1}}^{(\alpha(j+1), \ldots, \alpha+1))}\right| \\
& =\left|H_{k_{0}, \ldots, k_{L}}^{(\alpha(1), \ldots, \alpha(L+1))}-H_{k_{0}, \ldots, k_{j-1}}^{(\alpha(1), \ldots, \alpha(j))} H_{k_{j}, \ldots, k_{L}}^{(\alpha(j+1), \ldots, \alpha(L+1))}\right|
\end{aligned}
$$


with $k_{i}:=m_{i+1}-m_{i}$. Indeed, we have

$$
\begin{aligned}
C_{H}^{(j)}\left(m_{1}, \ldots, m_{L+1}\right) & =\left|\operatorname{Cov}\left(\prod_{i=1}^{j} H^{(\alpha(i))} \circ \sigma^{k_{i-1}} ; \prod_{l=1}^{L+1-j} H^{(\alpha(j+l))} \circ \sigma^{\left(k_{j+l-1}-k_{j}\right)+\left(k_{j}\right)}\right)\right| \\
& \leq M^{L+1} C_{r} \alpha_{r} k_{j}-r \max \left(k_{j-1}, k_{L}-k_{j}\right) \\
& \leq M^{L+1} C_{r} \alpha_{r} \frac{N^{\beta}}{4^{L}}
\end{aligned}
$$

- Let us suppose that $j$ is odd. Then, we have

$$
\frac{1}{N^{\frac{L+1}{2}}} \sum_{l \in E_{\beta, j}^{(L+1)}} \sup _{H \in \mathcal{E}_{L+1, M_{\infty}, M}}\left|H_{m_{1}, \ldots, m_{j}}^{(\alpha(1), \ldots, \alpha(j))}\right| \cdot\left|H_{m_{j+1}, \ldots, m_{L+1}}^{(\alpha(j+1), \ldots, \alpha(L+1))}\right| \leq(L+1) ! \frac{S\left(E^{(j)}\right)}{N^{\frac{j}{2}}} \frac{S\left(E^{(L+1-j)}\right)}{N^{\frac{L+1-j}{2}}}
$$

and, by induction hypothesis, we have $\lim _{N \rightarrow+\infty} \frac{S\left(E^{(j)}\right)}{N^{\frac{j}{2}}}=0$ and $\lim _{N \rightarrow+\infty} \frac{S\left(E^{(L+1-j)}\right)}{N^{\frac{L+1-j}{2}}}=0$. On the other hand, we have:

$$
\begin{aligned}
\frac{1}{N^{\frac{L+1}{2}}} \sum_{1 \in E_{\beta, j}^{(L+1)}} \sup _{H \in \mathcal{E}_{L+1, M_{\infty}, M}} \prod_{i=1}^{\frac{L+1}{2}}\left|H_{m_{2 i-1}, m_{2 i}}^{(\alpha(2 i-1), \alpha(2 i))}\right| & \leq \frac{1}{N^{\frac{L+1}{2}}} M_{\infty}^{L-1} \sum_{1 \in E_{\beta, j}^{(L+1)}} \sup _{H \in \mathcal{E}_{L+1, M_{\infty}, M}}\left|H_{m_{j}, m_{j+1}}^{(\alpha(j), \alpha(j+1))}\right| \\
& \leq \frac{1}{N^{\frac{L+1}{2}}} M_{\infty}^{L-1} \sum_{1 \in E_{\beta, j}^{(L+1)}} C_{r} M^{2} \alpha_{r} \frac{N^{\beta}}{4 L} \\
& \leq C_{r} M_{\infty}^{L-1} M^{2} N^{\frac{L+1}{2}} \alpha_{r} \frac{N^{\beta}}{4 L}
\end{aligned}
$$

Thus, we have $\lim _{N \rightarrow+\infty} \frac{S_{0}\left(E_{\beta, j}^{(L+1)}\right)}{N^{\frac{L+1}{2}}}=0$.

- Let us suppose that $j$ is even. Then, we have

$$
\begin{array}{r}
\frac{1}{N^{\frac{L+1}{2}}} \sum_{\mathbf{1} \in E_{\beta, j}^{(L+1)}} \sup _{H \in \mathcal{E}_{L+1, M_{\infty}, M}}\left|H_{m_{1}(\mathbf{l}), \ldots, m_{j}(\mathbf{l})}^{(\alpha(1), \ldots, \alpha(j))}-\prod_{i=1}^{\frac{j}{2}} H_{m_{2 i-1}(\mathbf{l}), m_{2 i}(\mathbf{l})}^{(\alpha(2 i-1), \alpha(2 i)}\right| \cdot\left|H_{m_{j+1}, \ldots, m_{L+1}}^{(\alpha(j+1), \ldots, \alpha(L+1))}\right| \\
\leq(L+1) ! \frac{S_{0}\left(E^{(j)}\right)}{N^{\frac{j}{2}}} \frac{S\left(E^{(L+1-j)}\right)}{N^{\frac{L+1-j}{2}}}
\end{array}
$$

Now, according to the foregoing and to the induction hypothesis, we have $\lim _{N \rightarrow+\infty} \frac{S_{0}\left(E^{(j)}\right)}{N^{\frac{j}{2}}}=0$ and $\sup _{N \geq 1} \frac{S\left(E^{(L+1-j)}\right)}{N^{\frac{L+1-j}{2}}}<+\infty$. In the same way, we get the same kind of inequality for the following quantity

$$
\frac{1}{N^{\frac{L+1}{2}}} \sum_{\mathbf{l} \in E_{\beta, j}^{(L+1)}} \sup _{H \in \mathcal{E}_{L+1, M_{\infty}, M}}\left|\prod_{i=1}^{\frac{j}{2}} H_{m_{2 i-1}(\mathbf{l}), m_{2 i}(\mathbf{l})}^{(\alpha(2 i-1),(2 i))}\right| \cdot\left|H_{m_{j+1}(\mathbf{l}), \ldots, m_{L+1}(\mathbf{l})}^{(\alpha(j+1), \ldots, \alpha(L+1))}-\prod_{i=\frac{j}{2}+1}^{\frac{L+1}{2}} H_{m_{2 i-1}(\mathbf{l}), m_{2 i}(\mathbf{l})}^{(\alpha(2 i-1), \alpha(2 i))}\right| .
$$

Thus, we have $\lim _{N \rightarrow+\infty} \frac{S_{0}\left(E_{\beta, j}^{(L+1)}\right)}{N^{\frac{L+1}{2}}}=0$. 
We conclude that we have $\lim _{N \rightarrow+\infty} \frac{S_{0}\left(E^{(L+1)}\right)}{N^{\frac{L+1}{2}}}=0$.

Proof of Proposition 2.3.5. We shall prove that, for any $\beta \in \mathbf{R}^{d}$, the family of random variables $\left(\left\langle\beta, V_{t}^{\varepsilon}(x, \cdot)-V_{s}^{\varepsilon}(x, \cdot)\right\rangle\right)_{\varepsilon>0}$ converges in distribution (for $\nu$ ) to $\langle\beta, N\rangle$ when $\varepsilon$ goes to 0 . Let $\beta$ be a vector in $\mathbf{R}^{d}$. According to Lemma 2.3.6, it is enough to prove that for any integer $p \geq 1$, we have

$$
\lim _{\varepsilon \rightarrow 0} \mathbf{E}_{\nu}\left[\left(\left\langle\beta, V_{t}^{\varepsilon}(x, \cdot)-V_{s}^{\varepsilon}(x, \cdot)\right\rangle\right)^{p}\right]=\mathbf{E}\left[(\langle\beta, N\rangle)^{p}\right]
$$

According to the foregoing, for any integer $L \geq 0$, we have

$$
\lim _{\varepsilon \rightarrow 0} \mathbf{E}_{\nu}\left[\left(\left\langle\beta, V_{t}^{\varepsilon}(x, \cdot)-V_{s}^{\varepsilon}(x, \cdot)\right\rangle\right)^{2 L+1}\right]=0 .
$$

Thus, it remains to show that, for any integer $L \geq 1$, we have

$$
\begin{aligned}
\lim _{\varepsilon \rightarrow 0} \mathbf{E}_{\nu}\left[\left(\left\langle\beta, V_{t}^{\varepsilon}(x, \cdot)-V_{s}^{\varepsilon}(x, \cdot)\right\rangle\right)^{2 L}\right] & =1 \cdot 3 \cdots(2 L-1)\left(\int_{s}^{t} \beta^{T} \cdot A\left(w_{u}(x)\right) \cdot \beta \mathrm{d} u\right)^{L} \\
& =\frac{(2 L) !}{(L) ! 2^{L}}\left(\int_{s}^{t} \beta^{T} \cdot A\left(w_{u}(x)\right) \cdot \beta \mathrm{d} u\right)^{L}
\end{aligned}
$$

where $A(X)$ is the $d$-dimensional matrix, the coefficients of which are the $a_{i, j}(X)$ defined in Proposition 2.2.2. Let us consider $g(X, \omega):=\langle\beta, G(X, \omega)\rangle$. Then we have

$$
\begin{aligned}
\mathbf{E}_{\nu}\left[\left(\left\langle\beta, V_{t}^{\varepsilon}(x, \cdot)-V_{s}^{\varepsilon}(x, \cdot)\right\rangle\right)^{2 L}\right] & =\mathbf{E}_{\nu}\left[\left(\left\langle\beta, \sqrt{\varepsilon} \int_{\frac{s}{\varepsilon}}^{\frac{t}{\varepsilon}} G\left(w_{\varepsilon u}(x), \sigma^{\lfloor u\rfloor}(\cdot)\right) \mathrm{d} u\right\rangle\right)^{2 L}\right] \\
& =\varepsilon^{L} \int_{\left[\frac{s}{\varepsilon} ; \frac{t}{\varepsilon}\right]^{2 L}} \mathbf{E}_{\nu}\left[\prod_{i=1}^{2 L} g\left(w_{\varepsilon u_{i}}(x), \sigma^{\left\lfloor u_{i}\right\rfloor}(\cdot)\right)\right] \mathrm{d} u_{1} \ldots \mathrm{d} u_{2 L} .
\end{aligned}
$$

According to Lemma 2.3.7, we have

$$
\lim _{\varepsilon \rightarrow 0} \mathbf{E}_{\nu}\left[\left(\left\langle\beta, V_{t}^{\varepsilon}(x, \cdot)-V_{s}^{\varepsilon}(x, \cdot)\right\rangle\right)^{2 L}\right]-A_{\varepsilon}=0,
$$

with

$$
A_{\varepsilon}:=\varepsilon^{L} \int_{\left[\frac{s}{\varepsilon} ; \frac{t}{\varepsilon}\right]^{2 L}} \prod_{i=1}^{L} \mathbf{E}_{\nu}\left[g\left(w_{\varepsilon u_{\alpha(2 i-1)}}(x), \sigma^{m_{2 i-1}}(\cdot)\right) \cdot g\left(w_{\varepsilon u_{\alpha(2 i)}}(x), \sigma^{m_{2 i}}(\cdot)\right)\right] \mathrm{d} u_{1} \ldots \mathrm{d} u_{2 L}
$$

where $\alpha(j)$ is the index of the $j^{\text {th }}$ smallest component of the integer vector $\left(\left\lfloor u_{1}\right\rfloor, \ldots,\left\lfloor u_{2 L}\right\rfloor\right)$ and $m_{i}:=\left\lfloor u_{\alpha(i)}\right\rfloor$, as in the proof of Lemma 2.3.4. We have

$$
A_{\varepsilon}=\varepsilon^{L} \int_{\left[\frac{s}{\varepsilon} ; \frac{t}{\varepsilon}\right]^{2 L}} \kappa_{\left\lfloor u_{1}\right\rfloor, \ldots,\left\lfloor u_{2 L}\right\rfloor} H^{\varepsilon}\left(u_{1}, \ldots, u_{2 L}\right) \mathrm{d} u_{1} \ldots \mathrm{d} u_{2 L},
$$

where we denote $\kappa_{n_{1}, \ldots, n_{2 L}}:=\#\left\{\mathbf{l} \in \mathbf{N}^{2 L}: \forall j=1, \ldots, 2 L ; m_{j}(\mathbf{l})=n_{j}\right\}$ and

$$
H^{\varepsilon}\left(u_{1}, \ldots, u_{2 L}\right):=\prod_{i=1}^{L} \mathbf{E}_{\nu}\left[g\left(w_{\varepsilon u_{2 i-1}}(x), \sigma^{\left\lfloor u_{2 i-1}\right\rfloor}(\cdot)\right) \cdot g\left(w_{\varepsilon u_{2 i}}(x), \sigma^{\left\lfloor u_{2 i}\right\rfloor}(\cdot)\right)\right] .
$$


Let $\gamma$ be a real number in $] \frac{L}{L+1} ; 1\left[\right.$. We denote $n_{\varepsilon}:=\left\lfloor\varepsilon^{-\gamma}\right\rfloor$ and $s_{k}=s_{k, \varepsilon}:=\frac{s}{\varepsilon}+k \frac{t-s}{\varepsilon n_{\varepsilon}}$. We have

$$
A_{\varepsilon}=\varepsilon^{L} \sum_{k_{1}, \ldots, k_{2 L}=0}^{n_{\varepsilon}-1} D_{k_{1}, \ldots, k_{2 L}}
$$

with $D_{k_{1}, \ldots, k_{2 L}}:=\int_{s_{k_{1}}}^{s_{k_{1}+1}} \ldots \int_{s_{k_{2 L}}}^{s_{k_{2}+1}} \kappa_{\left\lfloor u_{1}\right\rfloor, \ldots,\left\lfloor u_{2 L}\right\rfloor} H^{\varepsilon}\left(u_{1}, \ldots, u_{2 L}\right) \mathrm{d} u_{1} \ldots \mathrm{d} u_{2 L}$. Let $i=1, \ldots, L$. We have

$$
\left|\varepsilon^{L} \sum_{\mathbf{k}: k_{2 i-1}<k_{2 i}} D_{k_{1}, \ldots, k_{2 L}}\right| \leq(2 L) ! \varepsilon \sum_{k=0}^{n_{\varepsilon}-1} \int_{s_{k}}^{s_{k+1}} \int_{\frac{s}{\varepsilon}}^{s_{k}}\left|H^{\varepsilon}\left(u_{1}, u_{2}\right)\right| \mathrm{d} u_{1} \mathrm{~d} u_{2} \cdot\left(\varepsilon \int_{\frac{s}{\varepsilon}}^{\frac{t}{\varepsilon}} \int_{\frac{s}{\varepsilon}}^{\frac{t}{\varepsilon}}\left|H^{\varepsilon}\left(v_{1}, v_{2}\right)\right| \mathrm{d} v_{1} \mathrm{~d} v_{2}\right)^{L-1}
$$

where $\sum_{\mathbf{k}: k_{2 i-1<k_{2 i}}}$ means that the sum is taken over the $\mathbf{k}=\left(k_{1}, \ldots, k_{2 l}\right) \in\left\{0, \ldots, n_{\varepsilon}-1\right\}^{L}$ satisfying $k_{2 i-1}<k_{2 i}$. Now, as in the proof of Proposition 2.2.3 (see [10]), we get

$$
\varepsilon \sum_{k=0}^{n_{\varepsilon}-1} \int_{s_{k}}^{s_{k+1}} \int_{\frac{s}{\varepsilon}}^{s_{k}}\left|H^{\varepsilon}\left(u_{1}, u_{2}\right)\right| \mathrm{d} u_{1} \mathrm{~d} u_{2}=O\left(\varepsilon n_{\varepsilon}\right)
$$

On the other hand, according to Lemma 2.3.4, we have $\sup _{\varepsilon>0} \varepsilon \int_{\frac{s}{\varepsilon}}^{\frac{t}{\varepsilon}} \int_{\frac{s}{\varepsilon}}^{\frac{t}{\varepsilon}}\left|H^{\varepsilon}\left(v_{1}, v_{2}\right)\right| \mathrm{d} v_{1} \mathrm{~d} v_{2}<+\infty$. So, we have $\lim _{\varepsilon \rightarrow 0} \varepsilon^{L} \sum_{\mathbf{k}: k_{2 i-1}<k_{2 i}} D_{k_{1}, \ldots, k_{2 L}}=0$ (since we have $\lim _{\varepsilon \rightarrow 0} \varepsilon n_{\varepsilon}=0$ ). In the same way, we get $\lim _{\varepsilon \rightarrow 0} \varepsilon^{L} \sum_{\mathbf{k}: k_{2 i-1}>k_{2 i}} D_{k_{1}, \ldots, k_{2 L}}=0$. Thus, we have

$$
\lim _{\varepsilon \rightarrow 0} \varepsilon^{L} \sum_{\mathbf{k}: k_{2 i-1} \neq k_{2 i}} D_{k_{1}, \ldots, k_{2 L}}=0
$$

Therefore, we have

$$
A_{\varepsilon}=\varepsilon^{L} \sum_{k_{1}, \ldots, k_{L}=0}^{n_{\varepsilon}-1} D_{k_{1}, k_{1}, k_{2}, k_{2}, \ldots, k_{L}, k_{L}}+\epsilon_{0}^{\prime}(\varepsilon)
$$

with $\lim _{\varepsilon \rightarrow 0} \epsilon_{0}^{\prime}(\varepsilon)=0$. Let us define the set $E_{\varepsilon, L}$ of integer vectors $\mathbf{k}=\left(k_{1}, \ldots, k_{L}\right) \in\left\{0, \ldots, n_{\varepsilon}-1\right\}^{L}$ satisfying $k_{i} \neq k_{j}$ and $k_{i} \neq k_{j}+1$ for any integers $i, j=1, \ldots, L$ pairwise distinct. We have

$$
A_{\varepsilon}=\varepsilon^{L} \sum_{\mathbf{k} \in E_{\varepsilon, L}} D_{k_{1}, k_{1}, k_{2}, k_{2}, \ldots, k_{L}, k_{L}}+\epsilon_{1}^{\prime}(\varepsilon),
$$

with $\lim _{\varepsilon \rightarrow 0} \epsilon_{1}^{\prime}(\varepsilon)=0$. Indeed, for any integers $i, j=1, \ldots, L$ pairwise distinct, we have

$$
\begin{aligned}
\varepsilon^{L} \sum_{k_{1}, \ldots, k_{L}: k_{i} \in\left\{k_{j}, k_{j}+1\right\}}\left|D_{k_{1}, k_{1}, k_{2}, k_{2}, \ldots, k_{L}, k_{L}}\right| & \leq 2(2 L) ! \varepsilon^{L} n_{\varepsilon}{ }^{L-1} \frac{(t-s)^{2 L}}{\varepsilon^{2 L} n_{\varepsilon}{ }^{2 L}}\|g\|_{\infty}^{2 L} \\
& \leq 2(2 L) ! \frac{(t-s)^{2 L}}{\varepsilon^{L} n_{\varepsilon}{ }^{L+1}}\|g\|_{\infty}^{2 L}
\end{aligned}
$$

and $\lim _{\varepsilon \rightarrow 0} \varepsilon^{-L} n_{\varepsilon}-(L+1)=0$. Now, we suppose that the real number $\varepsilon>0$ is small enough such that we have $\varepsilon n_{\varepsilon}<t-s$. Let $\mathbf{k}=\left(k_{1}, \ldots, k_{L}\right)$ be in $E_{\varepsilon, L}$ and $\left(u_{1}, \ldots, u_{2 L}\right)$ be a vector in $\mathbf{R}^{2 L}$ such that $s_{k_{i}} \leq u_{2 i-1}, u_{2 i} \leq s_{k_{i}+1}$ for any integer $i=1, \ldots, L$. Let us observe that if we have $k_{i} \geq k_{i+1}$ (for some integer $i=1, \ldots, L-1$ ), then we 
have $k_{i} \geq k_{i+1}+2$ and so

$$
u_{2 i}-u_{2 i+1} \geq s_{k_{i}}-s_{k_{i+1}+1} \geq s_{k_{i+1}+2}-s_{k_{i+1}+1}=\frac{t-s}{\varepsilon n_{\varepsilon}} \geq 1 \text { and } \kappa_{\left\lfloor u_{1}\right\rfloor, \ldots,\left\lfloor u_{2 L}\right\rfloor}=0 .
$$

Therefore, we have:

$$
A_{\varepsilon}=\varepsilon^{L} \sum_{\mathbf{k} \in F_{\varepsilon}, L} D_{k_{1}, k_{1}, k_{2}, k_{2}, \ldots, k_{L}, k_{L}}+\epsilon_{1}^{\prime}(\varepsilon),
$$

with $\lim _{\varepsilon \rightarrow 0} \epsilon_{1}^{\prime}(\varepsilon)=0$, where $F_{\varepsilon, L}$ is the set of integer vectors $\mathbf{k}=\left(k_{1}, \ldots, k_{L}\right) \in\left\{0, \ldots, n_{\varepsilon}-1\right\}^{L}$ satisfying $k_{i}+1<k_{i+1}$ for any integer $i=1, \ldots, L-1$. Let $\mathbf{k}$ be in $F_{\varepsilon, L}$ and $\left(u_{1}, \ldots, u_{2 L}\right)$ be a vector in $\mathbf{R}^{2 L}$ such that $s_{k_{i}} \leq u_{2 i-1}, u_{2 i} \leq s_{k_{i}+1}$ for any integer $i=1, \ldots, L-1$. If we have $\left\lfloor u_{1}\right\rfloor \leq\left\lfloor u_{2}\right\rfloor \leq \cdots \leq\left\lfloor u_{2 L}\right\rfloor$, then we have $\kappa_{\left\lfloor u_{1}\right\rfloor, \ldots,\left\lfloor u_{2 L}\right\rfloor}=\frac{(2 L) !}{2^{m}}$, where $m$ is the number of integers $i=1, \ldots, L$ such that $\left\lfloor u_{2 i-1}\right\rfloor=\left\lfloor u_{2 i}\right\rfloor$; otherwise, we have $\kappa_{\left\lfloor u_{1}\right\rfloor, \ldots,\left\lfloor u_{2 L}\right\rfloor}=0$. Therefore, we have

$$
\varepsilon^{L} \sum_{\mathbf{k} \in F_{\varepsilon, L}} D_{k_{1}, k_{1}, k_{2}, k_{2}, \ldots, k_{L}, k_{L}}=(2 L) ! \varepsilon^{L} \sum_{\mathbf{k} \in F_{\varepsilon, L}} \prod_{i=1}^{L}\left(\int_{s_{k_{i}}}^{s_{k_{i}+1}} \int_{s_{k_{i}}}^{s_{k_{i}+1}} \alpha(u, v) H^{\varepsilon}(u, v) \mathrm{d} u \mathrm{~d} v\right)
$$

with $\alpha(u, v)=0$ if $\lfloor v\rfloor<\lfloor u\rfloor, \alpha(u, v)=1$ if $\lfloor u\rfloor<\lfloor v\rfloor$ and $\alpha(u, v)=\frac{1}{2}$ if $\lfloor u\rfloor=\lfloor v\rfloor$. Since $H^{\varepsilon}(v, u)=H^{\varepsilon}(u, v)$, we have

$$
\begin{aligned}
\varepsilon^{L} \sum_{\mathbf{k} \in F_{\varepsilon, L}} D_{k_{1}, k_{1}, k_{2}, k_{2}, \ldots, k_{L}, k_{L}}=\frac{(2 L) !}{2^{L}} \varepsilon^{L} \sum_{\mathbf{k} \in F_{\varepsilon, L}} \prod_{i=1}^{L}\left(\int_{s_{k_{i}}}^{s_{k_{i}+1}} \int_{s_{k_{i}}}^{s_{k_{i}+1}} H^{\varepsilon}\left(u_{1}, u_{2}\right) \mathrm{d} u_{1} \mathrm{~d} u_{2}\right) \\
=\frac{(2 L) !}{2^{L}} \varepsilon^{L} \sum_{k_{1}<\cdots<k_{L}} \prod_{i=1}^{L}\left(\int_{s_{k_{i}}}^{s_{k_{i}+1}} \int_{s_{k_{i}}}^{s_{k_{i}+1}} H^{\varepsilon}\left(u_{1}, u_{2}\right) \mathrm{d} u_{1} \mathrm{~d} u_{2}\right)+\epsilon_{2}^{\prime}(\varepsilon) \\
=\frac{(2 L) !}{L ! 2^{L}} \varepsilon^{L} \sum_{k_{1}, \ldots, k_{L}}^{L}: \operatorname{pairwise} \operatorname{distinct} \prod_{i=1}^{L}\left(\int_{s_{k_{i}}}^{s_{k_{i}+1}} \int_{s_{k_{i}}}^{s_{k_{i}+1}} H^{\varepsilon}\left(u_{1}, u_{2}\right) \mathrm{d} u_{1} \mathrm{~d} u_{2}\right)+\epsilon_{2}^{\prime}(\varepsilon) \\
=\frac{(2 L) !}{L ! 2^{L}} \varepsilon^{L} \sum_{k_{1}, \ldots, k_{L}=0}^{n_{\varepsilon}-1} \prod_{i=1}^{L}\left(\int_{s_{k_{i}}}^{s_{k_{i}+1}} \int_{s_{k_{i}}}^{s_{k_{i}+1}} H^{\varepsilon}\left(u_{1}, u_{2}\right) \mathrm{d} u_{1} \mathrm{~d} u_{2}\right)+\epsilon_{2}^{\prime}(\varepsilon)+\epsilon_{3}^{\prime}(\varepsilon) \\
=\frac{(2 L) !}{L ! 2^{L}}\left(\varepsilon \sum_{k=0}^{n_{\varepsilon}-1} \int_{s_{k}}^{s_{k+1}} \int_{s_{k}}^{s_{k+1}} H^{\varepsilon}(u, v) \mathrm{d} u \mathrm{~d} v\right)^{L}+\epsilon_{2}^{\prime}(\varepsilon)+\epsilon_{3}^{\prime}(\varepsilon),
\end{aligned}
$$

with $\left|\epsilon_{2}^{\prime}(\varepsilon)\right| \leq(L-1) \frac{(2 L) !}{2^{L}} \frac{(t-s)^{2 L}}{\varepsilon^{L} n_{\varepsilon} L+1}\|g\|_{\infty}^{2 L}$ and $\left|\epsilon_{3}^{\prime}(\varepsilon)\right| \leq L(L-1) \frac{(2 L) !}{L ! 2^{L}} \frac{(t-s)^{2 L}}{\varepsilon^{L} n_{\varepsilon} L+1}\|g\|_{\infty}^{2 L}$. Then we have $\lim _{\varepsilon \rightarrow 0} \epsilon_{2}^{\prime}(\varepsilon)+$ $\epsilon_{3}^{\prime}(\varepsilon)=0$. Now, according to the end of the proof of Proposition 2.2.3, we know that we have

$$
\lim _{\varepsilon \rightarrow 0} \varepsilon \sum_{k=0}^{n_{\varepsilon}-1} \int_{s_{k}}^{s_{k+1}} \int_{s_{k}}^{s_{k+1}} H^{\varepsilon}(u, v) \mathrm{d} u \mathrm{~d} v=\int_{s}^{t} \beta^{T} \cdot A\left(w_{u}(x)\right) \cdot \beta \mathrm{d} u .
$$

End of the proof of Theorem 2.3.2. We show that $\left(\left(V_{t}^{\varepsilon}(x, \cdot)\right)_{t}\right)_{\varepsilon}$ converges to $\left(V_{t}(x, \cdot)\right)_{t}$ in the sense of finite distributions when $\varepsilon$ goes to 0 .

1. We prove that, for any integer $p \geq 1$, any integers $n_{1}, \ldots, n_{p} \geq 1$, any vectors $\beta^{(1)}, \ldots, \beta^{(p)}$ in $\mathbf{R}^{d}$ and all real numbers $0<s_{1}<t_{1}<s_{2}<t_{2}<\cdots<s_{p}<t_{p}<T_{0}$, we have

$$
\lim _{\varepsilon \rightarrow 0} \mathbf{E}_{\nu}\left[\prod_{j=1}^{p}\left(\left\langle\beta^{(j)} ; V_{t_{j}}^{\varepsilon}(x, \cdot)-V_{s_{j}}^{\varepsilon}(x, \cdot)\right\rangle\right)^{n_{j}}\right]-\prod_{j=1}^{p} \mathbf{E}_{\nu}\left[\left(\left\langle\beta^{(j)} ; V_{t_{j}}^{\varepsilon}(x, \cdot)-V_{s_{j}}^{\varepsilon}(x, \cdot)\right\rangle\right)^{n_{j}}\right]=0 .
$$


Let $p \geq 1$ be an integer, $n_{1}, \ldots, n_{p} \geq 1$ be $p$ integers, $\beta^{(1)}, \ldots, \beta^{(p)}$ be in $\mathbf{R}^{d}$ and $s_{1}, \ldots, s_{p}, t_{1}, \ldots, t_{p}$ be real numbers satisfying $0<s_{1}<t_{1}<s_{2}<t_{2}<\cdots<s_{p}<t_{p}<T_{0}$, We write $N_{0}:=0, N_{j}:=n_{1}+\ldots+n_{j}$ (for any $j=1, \ldots, p)$ and $F^{\left(N_{1}, \ldots, N_{p}\right)}:=\prod_{i=1}^{p}\left\{\left\lfloor\frac{s_{i}}{\varepsilon}\right\rfloor, \ldots,\left\lfloor\frac{t_{i}}{\varepsilon}\right\rfloor\right\}^{n_{i}}$. Let us consider a real number $\varepsilon$ satisfying $0<\varepsilon<\frac{1}{2} \min _{j=1, \ldots, p-1}\left(s_{j+1}-t_{j}\right)$. For any integer $i=1, \ldots, N_{p}$ and any $\mathbf{l}=\left(l_{1}, \ldots, l_{N_{p}}\right)$ in $F^{\left(N_{1}, \ldots, N_{p}\right)}$, we denote by $\alpha(i)=\alpha(i)(\mathbf{l})$ the index of the $i^{\text {th }}$ smallest integer $l_{k}$ and $m_{i}:=l_{\alpha(i)}$ the $i^{\text {th }}$ smallest integer $l_{k}$. It is obvious that, for any $\mathbf{l}$ in $F^{\left(N_{1}, \ldots, N_{p}\right)}$ and any integer $j=1, \ldots, p$, the map $\alpha: i \mapsto \alpha(i)$ defines a bijection on the set $\left\{N_{j-1}+1, \ldots, N_{j}\right\}$. So, it is enough to show that we have

$$
\lim _{\varepsilon \rightarrow 0} \varepsilon^{\frac{N_{p}}{2}} \sum_{\mathbf{l} \in F^{\left(N_{1}, \ldots, N_{p}\right)}} \int_{m_{1}}^{m_{1}+1} \cdots \int_{m_{N_{p}}}^{m_{N_{p}}+1}\left|H_{u_{1}, \ldots, u_{N_{p}}}^{\varepsilon ; \alpha(1), \ldots, \alpha\left(N_{p}\right)}-\prod_{j=1}^{p} H_{u_{N_{j}-1}+1, \ldots, u_{N_{j}}}^{\varepsilon ; \alpha\left(N_{j}-1+1\right), \ldots, \alpha\left(N_{j}\right)}\right| \mathrm{d} u=0,
$$

with $\mathrm{d} u=\mathrm{d} u_{1} \ldots \mathrm{d} u_{N_{p}}$ and $H_{u_{1}, \ldots, u_{k}}^{\varepsilon ; n_{1}, \ldots, n_{k}}:=\mathbf{E}_{\nu}\left[\prod_{j=1}^{k}\left\langle\beta^{\left(i_{n_{j}}\right)}, G\left(w_{\varepsilon u_{j}}(x), \sigma^{\left\lfloor u_{j}\right\rfloor}(\cdot)\right)\right\rangle\right]$; where $i_{n}$ is the integer such that $N_{i_{n}-1}+1 \leq n \leq N_{i_{n}}$.

- First, let us observe that if $n_{i}$ is odd for some integer $i=1, \ldots, p$, then we have

$$
\lim _{\varepsilon \rightarrow 0} \varepsilon^{\frac{N_{p}}{2}} \sum_{\mathbf{l} \in F^{\left(N_{1}, \ldots, N_{p}\right)}} \int_{m_{1}}^{m_{1}+1} \cdots \int_{m_{N_{p}}}^{m_{N_{p}}+1} \prod_{j=1}^{p}\left|H_{u_{N_{j-1}+1}, \ldots, u_{N_{j}}}^{\varepsilon ; N_{j-1}+1, \ldots, N_{j}}\right| \mathrm{d} u_{1} \ldots \mathrm{d} u_{N_{p}}=0
$$

Indeed, we have

$$
\lim _{\varepsilon \rightarrow 0} \varepsilon^{\frac{n_{i}}{2}} \sum_{l_{1}, \ldots, l_{n_{i}}=\left\lfloor\frac{s_{i}}{\varepsilon}\right\rfloor}^{\left\lfloor\frac{t_{i}}{\varepsilon}\right\rfloor} \int_{m_{1}}^{m_{1}+1} \ldots \int_{m_{n_{i}}}^{m_{n_{i}}+1}\left|H_{u_{1}, \ldots, u_{n_{i}}}^{\varepsilon ; N_{i-1}+1, \ldots, N_{i}}\right| \mathrm{d} u_{1} \ldots \mathrm{d} u_{n_{i}}=0
$$

according to Lemma 2.3.7, and, for any $j \neq i$, we have

$$
\sup _{\varepsilon>0} \varepsilon^{\frac{n_{j}}{2}} \sum_{l_{1}, \ldots, l_{n_{j}}=\left\lfloor\frac{s_{j}}{\varepsilon}\right\rfloor}^{\left\lfloor\frac{t_{j}}{\varepsilon}\right\rfloor} \int_{m_{1}}^{m_{1}+1} \ldots \int_{m_{n_{j}}}^{m_{n_{j}}+1}\left|H_{u_{1}, \ldots, u_{n_{j}}}^{\varepsilon ; N_{j-1}+1, \ldots, N_{j}}\right| \mathrm{d} u_{1} \ldots \mathrm{d} u_{n_{j}}<+\infty
$$

according to Lemma 2.3.4.

- If $N_{p}$ is odd, according to Lemma 2.3.7, we have

$$
\lim _{\varepsilon \rightarrow 0} \varepsilon^{\frac{N_{p}}{2}} \sum_{l_{1}, \ldots, l_{N_{p}}=0}^{\left\lfloor\frac{t_{p}}{\varepsilon}\right\rfloor} \int_{m_{1}}^{m_{1}+1} \ldots \int_{m_{N_{p}}}^{m_{N_{p}}+1}\left|H_{u_{1}, \ldots, u_{N_{p}}}^{\varepsilon ; 1, \ldots, N_{p}}\right| \mathrm{d} u_{1} \ldots \mathrm{d} u_{N_{p}}=0
$$

- If $N_{p}$ is even, then, according to the Lemma 2.3.7, the following quantity converges to 0 when $\varepsilon$ goes to 0

$$
\varepsilon^{\frac{N_{p}}{2}} \sum_{\mathbf{l} \in F^{\left(N_{1}, \ldots, N_{p}\right)}} \int_{m_{1}}^{m_{1}+1} \cdots \int_{m_{N_{p}}}^{m_{N_{p}+1}}\left|H_{u_{1}, \ldots, u_{N_{p}}}^{\varepsilon ; \alpha(1), \ldots, \alpha\left(N_{p}\right)}-\prod_{j=1}^{\frac{N_{p}}{2}} H_{u_{2 j-1}, \alpha(2 j-1), \alpha(2 j)}^{\varepsilon ; u_{2 j}}\right| \mathrm{d} u_{1} \ldots \mathrm{d} u_{N_{p}} .
$$


- If $N_{p}$ is even and if one of the $n_{i}$ is odd, then there exists an index $i^{\prime}=1, \ldots, p$ such that $N_{i^{\prime}}$ is odd and, for any $\mathbf{l}$ in $F^{\left(N_{1}, \ldots, N_{p}\right)}$ and any $\left(u_{1}, \ldots, u_{N_{p}}\right) \in \mathbf{R}^{N_{p}}$ such that $\left\lfloor u_{i}\right\rfloor=m_{i}$ for any $i$, we have

$$
u_{\alpha\left(N_{i^{\prime}}\right)(\mathbf{l})} \leq \frac{t_{i^{\prime}}}{\varepsilon}+1 \leq \frac{s_{i^{\prime}+1}}{\varepsilon}-1 \leq u_{\alpha\left(N_{i^{\prime}}+1\right)(\mathbf{l})} .
$$

Therefore, we have

$$
\begin{gathered}
\varepsilon^{\frac{N_{p}}{2}} \sum_{\mathrm{l} \in F^{\left(N_{1}, \ldots, N_{p}\right)}} \int_{m_{1}}^{m_{1}+1} \cdots \int_{m_{N_{p}}}^{m_{N_{p}}+1} \prod_{j=1}^{\frac{N_{p}}{2}} \mid H_{u_{2 j-1}, u_{2 j}}^{\varepsilon ; \alpha(2 j-1), \alpha(2 j) \mid} \mathrm{d} u_{1} \ldots \mathrm{d} u_{N_{p}} \\
\leq \varepsilon^{\frac{N_{p}}{2}}\left(\frac{t_{p}}{\varepsilon}\right)^{N_{p}}\left(\max _{j}\left\|\left\langle\beta^{(j)}, G\right\rangle\right\|_{\infty}\right)^{N_{p}-2} C_{r, 1,1}\left(\max _{j=i^{\prime}, i^{\prime}+1}\left\|\left\langle\beta^{(j)}, G\right\rangle\right\|\right)^{2} \alpha_{r, 1,1} \frac{s_{i^{\prime}-t_{i^{\prime}-1}-2}}{\varepsilon},
\end{gathered}
$$

since we have $\left|H_{u_{N_{i^{\prime}}}, u_{N_{i^{\prime}}+1}^{\varepsilon}, \alpha\left(N_{i^{\prime}}\right), \alpha\left(N_{i^{\prime}}+1\right)}\right| \leq C_{r, 1,1}\left(\max _{j=i^{\prime}, i^{\prime}+1}\left\|\left\langle\beta^{(j)}, G\right\rangle\right\|\right)^{2} \alpha_{r, 1,1} \frac{s_{i^{\prime}-t_{i^{\prime}-1^{\prime}}-2}}{\varepsilon}$.

- If all the $n_{i}$ 's (and then all the $N_{i}$ 's) are even, then the following quantity converges to 0 when $\varepsilon$ goes to 0 :

$$
\varepsilon^{\frac{N_{p}}{2}} \sum_{\mathbf{l} \in F^{\left(N_{1}, \ldots, N_{p}\right)}} \int_{I_{m_{1}} \times \ldots \times I_{m_{N}}}\left|\prod_{i=1}^{p} H_{u_{N_{i-1}+1}, \ldots, u_{N_{i}}}^{\varepsilon ; N_{i-1}+1, \ldots, N_{i}}-\prod_{i=1}^{p} \prod_{j=1}^{\frac{n_{i}}{2}} H_{u_{N_{i-1}+2 j-1}, u_{N_{i-1}+2 j}}^{\varepsilon ; \alpha\left(N_{i-1}+2 j-1\right), \alpha\left(N_{i-1}+2 j\right)}\right| \mathrm{d} u,
$$

with $I_{k}:=[k ; k+1]$ and $\mathrm{d} u=\mathrm{d} u_{1} \ldots \mathrm{d} u_{N_{p}}$. Indeed, according to Lemma 2.3.7, for any integer $i=1, \ldots, p$, the following quantity converges to 0 when $\varepsilon$ goes to 0 :

$$
\varepsilon^{\frac{n_{i}}{2}} \sum_{l_{1}, \ldots, l_{n_{i}}=\left\lfloor\frac{s_{i}}{\varepsilon}\right\rfloor}^{\left\lfloor\frac{t_{i}}{\varepsilon}\right\rfloor} \int_{m_{1}}^{m_{1}+1} \cdots \int_{m_{n_{i}}}^{m_{n_{i}}+1}\left|H_{u_{1}, \ldots, u_{n_{i}}}^{\varepsilon ; N_{i-1}+1, \ldots, N_{i}}-\prod_{j=1}^{\frac{n_{i}}{2}} H_{u_{2 j-1}, u_{2 j}}^{\varepsilon ; \alpha\left(N_{i-1}+2 j-1\right), \alpha\left(N_{i-1}+2 j\right)}\right| \mathrm{d} u
$$

with $\mathrm{d} u=\mathrm{d} u_{1} \ldots \mathrm{d} u_{n_{i}}$ and, according to Lemma 2.3.4, we have

$$
\sup _{\varepsilon>0} \varepsilon^{\frac{n_{i}}{2}} \sum_{l_{1}, \ldots, l_{n_{i}}=\left\lfloor\frac{s_{i}}{\varepsilon}\right\rfloor}^{\left\lfloor\frac{t_{i}}{\varepsilon}\right\rfloor} \int_{m_{1}}^{m_{1}+1} \cdots \int_{m_{n_{i}}}^{m_{n_{i}}+1}\left|H_{u_{1}, \ldots u_{n_{i}}}^{\varepsilon ; N_{i-1}+1, \ldots, N_{i}}\right| \mathrm{d} u_{1} \ldots \mathrm{d} u_{n_{i}}<+\infty .
$$

2. Therefore, for any integers $n \geq 1$ and $p \geq 1$ and any vectors $\beta^{(1)}, \ldots, \beta^{(p)}$ in $\mathbf{R}^{d}$, we have:

$$
\begin{aligned}
\left.\lim _{\varepsilon \rightarrow 0} \mathbf{E}_{\nu}\left[\left(\sum_{i=1}^{p}\left\langle\beta^{(i)} ;\left(V_{t_{i}}^{\varepsilon}-V_{s_{i}}^{\varepsilon}\right)(x, \cdot)\right)\right\rangle\right)^{n}\right] & \left.=\lim _{\varepsilon \rightarrow 0} \sum_{k_{1}+\cdots+k_{p}=n} \frac{n !}{k_{1} ! \ldots k_{p} !} \mathbf{E}_{\nu}\left[\prod_{i=1}^{p}\left(\left\langle\beta^{(i)} ;\left(V_{t_{i}}^{\varepsilon}-V_{s_{i}}^{\varepsilon}\right)(x, \cdot)\right)\right\rangle\right)^{k_{i}}\right] \\
& \left.=\lim _{\varepsilon \rightarrow 0} \sum_{k_{1}+\cdots+k_{p}=n} \frac{n !}{k_{1} ! \ldots k_{p} !} \prod_{i=1}^{p} \mathbf{E}_{\nu}\left[\left(\left\langle\beta^{(i)} ;\left(V_{t_{i}}^{\varepsilon}-V_{s_{i}}^{\varepsilon}\right)(x, \cdot)\right)\right\rangle\right)^{k_{i}}\right] \\
& =\sum_{k_{1}+\cdots+k_{p}=n} \frac{n !}{k_{1} ! \ldots k_{p} !} \prod_{i=1}^{p} \mathbf{E}\left[\left(\left\langle\beta^{(i)} ;\left(B_{t_{i}}-B_{s_{i}}\right)(x, \cdot)\right\rangle\right)^{k_{i}}\right] \\
& =\mathbf{E}\left[\left(\sum_{i=1}^{p}\left\langle\beta^{(i)} ;\left(B_{t_{i}}-B_{s_{i}}\right)(x, \cdot)\right\rangle\right)^{n}\right],
\end{aligned}
$$


where $B_{t}(x, \cdot)$ is a centered gaussian process with independent increments such that $\operatorname{Cov}\left(B_{t}(x, \cdot)\right)=$ $\int_{0}^{t} A\left(w_{u}(x)\right) \mathrm{d} u$, with $A(X)=\left(a_{i, j}(X)\right)_{i, j}$. Then, according to Lemma 2.3.6, the random variable $\left.\sum_{i=1}^{p}\left\langle\beta^{(i)} ;\left(V_{t_{i}}^{\varepsilon}-V_{s_{i}}^{\varepsilon}\right)(x, \cdot)\right)\right\rangle$ converges in distribution to $\left.\sum_{i=1}^{p}\left\langle\beta^{(i)} ; B_{t_{i}}(x, \cdot)-B_{s_{i}}(x, \cdot)\right)\right\rangle$.

\subsection{Convergence in distribution of $(e)$ (proof of Th. 2.1.3)}

In this paragraph, we adapt Khas'minskii's arguments to our situation. Following [10], we introduce the process $\left(y_{t}^{\varepsilon}(x, \cdot)\right)_{t}$ solution of the following differential equation:

$$
y_{t}^{\varepsilon}(x, \cdot)=v_{t}^{\varepsilon}(x, \cdot)+\int_{0}^{t} D \bar{F}\left(w_{s}(x)\right) \cdot y_{s}^{\varepsilon}(x, \cdot) \mathrm{d} s .
$$

We notice that we have

$$
y_{t}^{\varepsilon}(x, \cdot)=\int_{0}^{t} \mathrm{e}^{\int_{s}^{t} D \bar{F}\left(w_{u}(x)\right) \mathrm{d} u} \frac{\mathrm{d} v_{s}^{\varepsilon}(x, \cdot)}{\mathrm{d} s} \mathrm{~d} s=v_{t}^{\varepsilon}(x, \cdot)+\int_{0}^{t} K(s, t) v_{s}^{\varepsilon}(x, \cdot) \mathrm{d} s,
$$

with $K(s, t):=D \bar{F}\left(w_{s}(x)\right) \mathrm{e}^{\int_{s}^{t} D \bar{F}\left(w_{u}(x)\right) \mathrm{d} u}$. Let us consider the continuous Gaussian process $\left(v_{t}(x, \cdot)\right)_{t}($ limit of the family of processes $\left.\left(\left(v_{t}^{\varepsilon}(x, \cdot)\right)_{t}\right)_{\varepsilon>0}\right)$ and the process $\left(e_{t}^{0}(x, \cdot)\right)_{t}$ defined as in the statement of Theorem 2.1.3.

Proposition 2.4.1. Under Hypothesis 2.1.2, for any $x$ in $\mathbf{R}^{d},\left(\left(y_{t}^{\varepsilon}(x, \cdot), v_{t}^{\varepsilon}(x, \cdot)\right)_{0 \leq t \leq T_{0}}\right)_{\varepsilon>0}$ converges in distribution (for $\nu$ and for the uniform topology on $\left.C\left(\left[0, T_{0}\right]\right)\right)$ to $\left(e_{t}^{0}(x, \cdot), v_{t}(x, \cdot)\right)_{0 \leq t \leq T_{0}}$ when $\varepsilon$ goes to 0 .

Proof. Let $x$ be a point in $\mathbf{R}^{d}$. We start by proving the tightness of this family of processes, then we identify the cluster values. For any real number $t \in\left[0 ; T_{0}\right]$ and any $\omega \in \Omega$, we have

$$
\left|y_{t}^{\varepsilon}(x, \omega)\right| \leq\left|v_{t}^{\varepsilon}(x, \omega)\right|+\|D \bar{F}\|_{\infty} \mathrm{e}^{\|D \bar{F}\|_{\infty} t} \int_{0}^{t}\left|v_{s}^{\varepsilon}(x, \omega)\right| \mathrm{d} s .
$$

Thus, we have

$$
\sup _{\varepsilon>0} \sup _{t \in\left[0 ; T_{0}\right]}\left\|y_{t}^{\varepsilon}(x, \cdot)\right\|_{4}<+\infty
$$

Let a real number $t \in\left[0 ; T_{0}[\right.$ be given. Then, for any $\left.h \in] 0 ; T_{0}-t\right]$, we have

$$
\frac{1}{\sqrt{h}}\left|y_{t+h}^{\varepsilon}(x, \cdot)-y_{t}^{\varepsilon}(x, \cdot)\right| \leq \frac{1}{\sqrt{h}}\left|v_{t+h}^{\varepsilon}(x, \cdot)-v_{t}^{\varepsilon}(x, \cdot)\right|+\frac{1}{\sqrt{h}} \int_{t}^{t+h}\left|D \bar{F}\left(w_{s}(x)\right)\right| \cdot\left|y_{s}^{\varepsilon}(x, \cdot)\right| \mathrm{d} s .
$$

From which, we conclude that we have

$$
\frac{1}{\sqrt{h}}\left\|y_{t+h}^{\varepsilon}(x, \cdot)-y_{t}^{\varepsilon}(x, \cdot)\right\|_{4} \leq \frac{1}{\sqrt{h}}\left\|v_{t+h}^{\varepsilon}(x, \cdot)-v_{t}^{\varepsilon}(x, \cdot)\right\|_{4}+\|D \bar{F}\|_{\infty} \frac{1}{\sqrt{h}} \int_{t}^{t+h}\left\|y_{s}^{\varepsilon}(x, \cdot)\right\|_{4} \mathrm{~d} s
$$

So, the family of processes $\left(\left(y_{t}^{\varepsilon}(x, \cdot), v_{t}^{\varepsilon}(x, \cdot)\right)_{0 \leq t \leq T_{0}}\right)_{\varepsilon>0}$ is tight. Let $\left(y_{t}(x, \cdot), v_{t}(x, \cdot)\right)_{0 \leq t \leq T_{0}}$ be a cluster value of this family of processes for the convergence in distribution for $\nu$ and for the uniform topology on $C\left(\left[0, T_{0}\right]\right)$ (when $\varepsilon$ goes to 0 ). Then, the left member of the following equality:

$$
y_{t}^{\varepsilon}(x, \cdot)-v_{t}^{\varepsilon}(x, \cdot)-\int_{0}^{t} D \bar{F}\left(w_{s}(x)\right) \cdot y_{s}^{\varepsilon}(x, \cdot) \mathrm{d} s=0
$$


converges in distribution (when $\varepsilon$ goes to 0 ) to

$$
y_{t}(x, \cdot)-v_{t}(x, \cdot)-\int_{0}^{t} D \bar{F}\left(w_{s}(x)\right) \cdot y_{s}(x, \cdot) \mathrm{d} s .
$$

Therefore, we get $y_{t}(x, \cdot)=v_{t}(x, \cdot)+\int_{0}^{t} D \bar{F}\left(w_{s}(x)\right) \cdot y_{s}(x, \cdot) \mathrm{d} s$.

Now, we prove the main result of this section.

Proof of Theorem 2.1.3. The family of processes $\left(\left(\frac{e_{t}^{\varepsilon}(x, \cdot)}{\sqrt{\varepsilon}}\right)_{0 \leq t \leq T_{0}}\right)_{\varepsilon>0}$ is tight. Indeed, for any real numbers $t$ and $h$ satisfying $0 \leq t<t+h \leq T_{0}$, we have

$$
\frac{1}{\sqrt{h}} \frac{1}{\sqrt{\varepsilon}}\left|e_{t+h}^{\varepsilon}(x, \cdot)-e_{t}^{\varepsilon}(x, \cdot)\right| \leq \frac{1}{\sqrt{h}}\left|v_{t+h}^{\varepsilon}(x, \cdot)-v_{t}^{\varepsilon}(x, \cdot)\right|+\frac{\left\|D_{1} F\right\|_{\infty}}{\sqrt{h}} \int_{t}^{t+h} \frac{\left|e_{s}^{\varepsilon}(x, \cdot)\right|}{\sqrt{\varepsilon}} \mathrm{d} s
$$

and, according to the foregoing, we have

$$
\sup _{0 \leq t<t+h \leq T_{0}} \frac{1}{\sqrt{h}}\left\|v_{t+h}^{\varepsilon}(x, \cdot)-v_{t}^{\varepsilon}(x, \cdot)\right\|_{4}<+\infty \text { and } \sup _{s \in\left[0 ; T_{0}\right]}\left\|\frac{e_{s}^{\varepsilon}(x, \cdot)}{\sqrt{\varepsilon}}\right\|_{4}<\infty .
$$

Thus we have $\sup _{0 \leq t<t+h \leq T_{0}}\left\|\frac{1}{\sqrt{h}} \frac{1}{\sqrt{\varepsilon}}\left(e_{t+h}^{\varepsilon}(x, \cdot)-e_{t}^{\varepsilon}(x, \cdot)\right)\right\|_{4}<+\infty$. In order to identify the cluster values, we show that we have

$$
\sup _{0 \leq t \leq T_{0}}\left\|\frac{e_{t}^{\varepsilon}(x, \cdot)}{\sqrt{\varepsilon}}-y_{t}^{\varepsilon}(x, \cdot)\right\|_{1}=O\left(\varepsilon^{\frac{1}{4}}\right)
$$

- First, we show that we have $\sup _{0 \leq t \leq T_{0}}\left\|b_{t}^{\varepsilon}(x, \cdot)\right\|_{1}=O(\sqrt{\varepsilon})$, with

$$
b_{t}^{\varepsilon}(x, \omega):=\frac{1}{\sqrt{\varepsilon}} e_{t}^{\varepsilon}(x, \omega)-v_{t}^{\varepsilon}(x, \omega)-\frac{1}{\sqrt{\varepsilon}} \int_{0}^{t} D_{1} F\left(w_{s}(x), \sigma^{\left\lfloor\frac{s}{\varepsilon}\right\rfloor}(\omega)\right) \cdot e_{s}^{\varepsilon}(x, \omega) \mathrm{d} s .
$$

Indeed, we have

$$
\frac{1}{\sqrt{\varepsilon}} e_{t}^{\varepsilon}(x, \omega)-v_{t}^{\varepsilon}(x, \omega)=\frac{1}{\sqrt{\varepsilon}} \int_{0}^{t} F\left(x_{s}^{\varepsilon}(x, \omega), \sigma^{\left\lfloor\frac{s}{\varepsilon}\right\rfloor}(\omega)\right)-F\left(w_{s}(x), \sigma^{\left\lfloor\frac{s}{\varepsilon}\right\rfloor}(\omega)\right) \mathrm{d} s .
$$

Therefore, we have

$$
\left\|b_{t}^{\varepsilon}(x, \cdot)\right\|_{1} \leq \frac{1}{2} \frac{1}{\sqrt{\varepsilon}} \int_{0}^{t}\left\|D_{1}^{2} F\right\|_{\infty} \cdot\left\|x_{s}^{\varepsilon}(x, \cdot)-w_{s}(x)\right\|_{2}^{2} \mathrm{~d} s=\frac{1}{2} \sqrt{\varepsilon} \int_{0}^{t}\left\|D_{1}^{2} F\right\|_{\infty}\left\|\frac{e_{s}^{\varepsilon}(x, \cdot)}{\sqrt{\varepsilon}}\right\|_{2}^{2} \mathrm{~d} s .
$$

- Let us write $c_{t}^{\varepsilon}(x, \omega):=\frac{e_{t}^{\varepsilon}(x, \omega)}{\sqrt{\varepsilon}}-y_{t}^{\varepsilon}(x, \omega)$. Then, we have

$$
c_{t}^{\varepsilon}(x, \omega)=\frac{e_{t}^{\varepsilon}(x, \omega)}{\sqrt{\varepsilon}}-v_{t}^{\varepsilon}(x, \omega)-\int_{0}^{t} D \bar{F}\left(w_{s}(x)\right) \cdot y_{s}^{\varepsilon}(x, \omega) \mathrm{d} s .
$$

We have

$$
\left|c_{t}^{\varepsilon}(x, \cdot)-\int_{0}^{t} D_{1} F\left(w_{s}(x), \sigma^{\left\lfloor\frac{s}{\varepsilon}\right\rfloor}(\cdot)\right) c_{s}^{\varepsilon}(x, \cdot) \mathrm{d} s\right| \leq k_{t}^{\varepsilon}(x, \cdot),
$$


with $k_{t}^{\varepsilon}(x, \cdot):=\left|b_{t}^{\varepsilon}(x, \cdot)\right|+\left|\int_{0}^{t} D_{1} \tilde{F}\left(w_{s}(x), \sigma^{\left\lfloor\frac{s}{\varepsilon}\right\rfloor}(\cdot)\right) \cdot y_{s}^{\varepsilon}(x, \cdot) \mathrm{d} s\right|$. From this, we get

$$
\left|c_{t}^{\varepsilon}(x, \cdot)\right| \leq k_{t}^{\varepsilon}(x, \cdot)+\int_{0}^{t}\left\|D_{1} F\right\|_{\infty} \cdot\left|c_{s}^{\varepsilon}(x, \cdot)\right| \mathrm{d} s .
$$

According to Gronwall lemma, we have

$$
\left|c_{t}^{\varepsilon}(x, \cdot)\right| \leq k_{t}^{\varepsilon}(x, \cdot)+\int_{0}^{t} k_{s}^{\varepsilon}(x, \cdot)\left\|D_{1} F\right\|_{\infty} \mathrm{e}^{(t-s)\left\|D_{1} F\right\|_{\infty}} \mathrm{d} s .
$$

Thus, we have

$$
\left\|c_{t}^{\varepsilon}(x, \cdot)\right\|_{1} \leq\left(1+T_{0}\left\|D_{1} F\right\|_{\infty} \mathrm{e}^{T_{0}\left\|D_{1} F\right\|_{\infty}}\right) \sup _{0 \leq s \leq T_{0}}\left\|k_{s}^{\varepsilon}(x, \cdot)\right\|_{1}
$$

It remains to show that we have:

$$
\sup _{0 \leq t \leq T_{0}}\left\|\int_{0}^{t} D_{1} \tilde{F}\left(w_{s}(x), \sigma^{\left\lfloor\frac{s}{\varepsilon}\right\rfloor}(\cdot)\right) \cdot y_{s}^{\varepsilon}(x, \cdot) \mathrm{d} s\right\|_{1}=O\left(\varepsilon^{\frac{1}{4}}\right)
$$

Using the expression of $y_{t}^{\varepsilon}$ given before this proof, we observe that it suffices to show that, for any integers $j, k, l=1, \ldots, d$, the following quantities are in $O\left(\varepsilon^{\frac{1}{4}}\right)$ :

$$
\begin{aligned}
& A_{\varepsilon}(x):=\sup _{0 \leq t \leq T_{0}}\left\|\int_{0}^{t}\left(D_{1} \tilde{F}\right)_{k, j}\left(w_{s}(x), \sigma^{\left\lfloor\frac{s}{\varepsilon}\right\rfloor}(\cdot)\right) \cdot\left(v_{s}^{\varepsilon}(x, \cdot)\right)_{j} \mathrm{~d} s\right\|_{1}, \\
& B_{\varepsilon}(x):=\sup _{0 \leq t \leq T_{0}}\left\|\int_{0}^{t}\left(D_{1} \tilde{F}\right)_{k, j}\left(w_{s}(x), \sigma^{\left\lfloor\frac{s}{\varepsilon}\right\rfloor}(\cdot)\right) \int_{0}^{s} K_{j, l}(u, s)\left(v_{u}^{\varepsilon}(x, \cdot)\right)_{l} \mathrm{~d} u \mathrm{~d} s\right\|_{1} .
\end{aligned}
$$

To see this, we shall use the following equality:

$$
\left(D_{1} \tilde{F}\right)_{k, j}(x, \omega)=\frac{\tilde{F}_{k}\left(x+h \mathbf{e}_{j}, \omega\right)-\tilde{F}_{k}(x, \omega)}{h}+\delta_{k, j}(x, \omega, h),
$$

where $\left(\mathbf{e}_{1}, \ldots, \mathbf{e}_{d}\right)$ is the canonical basis of $\mathbf{R}^{d}$ and with $\left|\delta_{k, j}(x, \omega, h)\right| \leq \frac{\left\|D_{1}^{2} \tilde{F}\right\|_{\infty}}{2} h$. We take $h=\varepsilon^{\frac{1}{4}}$. Thus, we have

$$
A_{\varepsilon}(x) \leq a_{\varepsilon}(x)+b_{\varepsilon}(x)
$$

with

$$
\begin{aligned}
a_{\varepsilon}(x) & :=\sup _{0 \leq t \leq T_{0}}\left\|\int_{0}^{t} \delta_{k, j}\left(w_{s}(x), \sigma^{\left\lfloor\frac{s}{\varepsilon}\right\rfloor}(\cdot), h\right) \cdot\left(v_{s}^{\varepsilon}(x, \cdot)\right)_{j} \mathrm{~d} s\right\|_{1}, \\
& \leq \int_{0}^{T_{0}} \frac{\left\|D_{1}^{2} \tilde{F}\right\|_{\infty}}{2} h\left\|\left(v_{s}^{\varepsilon}(x, \cdot)\right)_{j}\right\|_{1} \mathrm{~d} s \\
& \leq T_{0} \frac{\left\|D_{1}^{2} \tilde{F}\right\|_{\infty}}{2} h \sup _{0 \leq t \leq T_{0}}\left\|\left(v_{t}^{\varepsilon}(x, \cdot)\right)_{j}\right\|_{1}=O\left(\varepsilon^{\frac{1}{4}}\right)
\end{aligned}
$$


and

$$
\begin{aligned}
b_{\varepsilon}(x) & :=\sup _{0 \leq t \leq T_{0}} \frac{1}{h}\left\|\int_{0}^{t} L_{k, j}\left(w_{s}(x), \sigma^{\left\lfloor\frac{s}{\varepsilon}\right\rfloor}(\cdot), h\right) \cdot\left(v_{s}^{\varepsilon}(x, \cdot)\right)_{j} \mathrm{~d} s\right\|_{1} \\
& =\sup _{0 \leq t \leq T_{0}} \frac{1}{h}\left\|\varepsilon \int_{0}^{\frac{t}{\varepsilon}} L_{k, j}\left(w_{\varepsilon s}(x), \sigma^{\lfloor s\rfloor}(\cdot), h\right) \sqrt{\varepsilon} \int_{0}^{s} \tilde{F}_{j}\left(w_{\varepsilon u}(x), \sigma^{\lfloor u\rfloor}(\cdot)\right) \mathrm{d} u \mathrm{~d} s\right\|_{2} \\
& \leq \frac{1}{h}\left(\varepsilon^{3} \sum_{n_{1}, n_{2}, n_{3}, n_{4}=0}^{\left\lfloor\frac{T_{0}}{\varepsilon}\right\rfloor} \int_{n_{1}}^{n_{1}+1} \int_{n_{2}}^{n_{2}+1} \int_{n_{3}}^{n_{3}+1} \int_{n_{4}}^{n_{4}+1}\left|\mathbf{E}_{\nu}\left[\prod_{i=1}^{4} G_{i}\left(w_{\varepsilon u_{i}}(x), \sigma^{\left\lfloor u_{i}\right\rfloor}(\cdot)\right)\right]\right| \mathrm{d} u\right)^{\frac{1}{2}},
\end{aligned}
$$

with $L_{k, j}(x, \omega, h):=\tilde{F}_{k}\left(x+h \mathbf{e}_{j}, \omega\right)-\tilde{F}_{k}(x, \omega), \mathrm{d} u=\mathrm{d} u_{1} \mathrm{~d} u_{2} \mathrm{~d} u_{3} \mathrm{~d} u_{4}$ and $G_{1}=G_{2}=L_{k, j}(\cdot, \cdot, h)$ and $G_{3}=G_{4}=\tilde{F}_{j}$. So, according to Hypothesis 2.1.2 and to Lemma 2.3 .4 with $L=4$ and $H^{(i)}=G_{i}\left(w_{\varepsilon u_{i}}(x), \cdot\right)$ (for $i=1, \ldots, 4$ ), we get

On the other hand, we have

$$
A_{\varepsilon}(x)=O\left(\varepsilon^{\frac{1}{4}}\right)
$$

$$
B_{\varepsilon}(x) \leq a_{\varepsilon}^{\prime}(x)+b_{\varepsilon}^{\prime}(x)
$$

with

$$
\begin{aligned}
a_{\varepsilon}^{\prime}(x) & :=\sup _{0 \leq t \leq T_{0}}\left\|\int_{0}^{t} \delta_{k, j}\left(w_{s}(x), \sigma^{\left\lfloor\frac{s}{\varepsilon}\right.}(\cdot), h\right) \int_{0}^{s} K_{j, l}(u, s)\left(v_{u}^{\varepsilon}(x, \cdot)\right)_{l} \mathrm{~d} u \mathrm{~d} s\right\|_{1} \\
& \leq h \cdot T_{0} \frac{\left\|D_{1}^{2} \tilde{F}\right\|_{\infty}}{2} T_{0}\|K\|_{\infty} \sup _{0 \leq t \leq T_{0}}\left\|\left(v_{t}^{\varepsilon}(x, \cdot)\right)_{l}\right\|_{1}=O\left(\varepsilon^{\frac{1}{4}}\right)
\end{aligned}
$$

and

$$
\begin{aligned}
b_{\varepsilon}^{\prime}(x) & :=\sup _{0 \leq t \leq T_{0}} \frac{1}{h}\left\|\int_{0}^{t} L_{k, j}\left(w_{s}(x), \sigma^{\left\lfloor\frac{s}{\varepsilon}\right.}(\cdot), h\right) \int_{0}^{s} K_{j, l}(u, s)\left(v_{u}^{\varepsilon}(x, \cdot)\right)_{l} \mathrm{~d} u \mathrm{~d} s\right\|_{1} \\
& \leq \sup _{0 \leq t \leq T_{0}} \frac{1}{h}\left\|\int_{0}^{t} L_{k, j}\left(w_{s}(x), \sigma^{\left\lfloor\frac{s}{\varepsilon}\right.}(\cdot), h\right) \int_{0}^{s} K_{j, l}(u, s) \sqrt{\varepsilon} \int_{0}^{\frac{u}{\varepsilon}} \tilde{F}_{l}\left(w_{\varepsilon v}(x), \sigma^{\lfloor v\rfloor}(\cdot)\right) \mathrm{d} v \mathrm{~d} u \mathrm{~d} s\right\|_{2} \\
& =\sup _{0 \leq t \leq T_{0}} \frac{1}{h}\left\|\varepsilon \sqrt{\varepsilon} \int_{0}^{\frac{t}{\varepsilon}} L_{k, j}\left(w_{\varepsilon s}(x), \sigma^{\lfloor s\rfloor}(\cdot)\right) \int_{0}^{\varepsilon s} K_{j, l}(u, \varepsilon s) \int_{0}^{\frac{u}{\varepsilon}} \tilde{F}_{l}\left(w_{\varepsilon v}(x), \sigma^{\lfloor v\rfloor}(\cdot)\right) \mathrm{d} v \mathrm{~d} u \mathrm{~d} s\right\|_{2} \\
& \leq \frac{\varepsilon^{\frac{3}{2}}}{h}\left(\sum_{n_{1}, \ldots, n_{4}=0}^{\left\lfloor\frac{T_{0}}{\varepsilon}\right\rfloor} T_{0}^{2}\|K\|_{\infty}^{2} \int_{n_{1}}^{n_{1}+1} \int_{n_{2}}^{n_{2}+1} \int_{n_{3}}^{n_{3}+1} \int_{n_{4}}^{n_{4}+1}\left|\mathbf{E}_{\nu}\left[\prod_{i=1}^{4} G_{i}\left(w_{\varepsilon u_{i}}(x), \sigma^{\left\lfloor u_{i}\right\rfloor}(\cdot)\right)\right]\right| \mathrm{d} u\right)^{\frac{1}{2}},
\end{aligned}
$$

with $\mathrm{d} u=\mathrm{d} u_{1} \mathrm{~d} u_{2} \mathrm{~d} u_{3} \mathrm{~d} u_{4}$ and $G_{1}=G_{2}=L_{k, j}(\cdot, \cdot, h)$ and $G_{3}=G_{4}=\tilde{F}_{l}$. So, we have

$$
B_{\varepsilon}(x)=O\left(\varepsilon^{\frac{1}{4}}\right)
$$

according to Lemma 2.3 .4 with $L=4$ and $H^{(i)}=G_{i}\left(w_{\varepsilon u_{i}}(x), \cdot\right)$ (for $\left.i=1, \ldots, 4\right)$. So, we have shown that we have:

$$
\left.\sup _{0 \leq t \leq T_{0}} \| \int_{0}^{t}\left(D_{1} \tilde{F}\left(w_{s}(x), \sigma^{\left\lfloor\frac{s}{\varepsilon}\right.}\right\rfloor(\cdot)\right)\right) \cdot y_{s}^{\varepsilon}(x, \cdot) \mathrm{d} s \|_{1}=O\left(\varepsilon^{\frac{1}{4}}\right) .
$$


- We get $\sup _{0 \leq t \leq T_{0}}\left\|c_{t}^{\varepsilon}(x, \cdot)\right\|_{L^{1}}=O\left(\varepsilon^{\frac{1}{4}}\right)$.

\section{Averaging method for a suspension flow: General Results}

\subsection{Hypothesis and main result}

Let $(\Omega, \nu, \sigma)$ be a probabilised dynamical system and $\tau: \Omega \rightarrow] 0 ;+\infty[$ be a measurable function such that $C^{-1} \leq \tau \leq C$ for some constant $C>0$. The suspension flow defined over $(\Omega, \nu, \sigma)$ by the function $\tau$ is the flow $\left(Y_{t}\right)_{t \geq 0}$ defined by $Y_{t}(\omega, s)=(\omega, t+s)$ on the set $\mathcal{M}:=\{(\omega, s): \omega \in \Omega, 0 \leq s \leq \tau(\omega)\}$ (with the identification $(\omega, \tau(\omega)) \equiv(\sigma(\omega), 0))$ endowed with the probability measure $\mu$ given by:

$$
\int_{\mathcal{M}} f(\omega, s) \mathrm{d} \mu(\omega, s)=\frac{1}{\int_{\Omega} \tau(\omega) \mathrm{d} \nu(\omega)} \int_{\Omega} \int_{0}^{\tau(\omega)} f(\omega, s) \mathrm{d} s \mathrm{~d} \nu(\omega)
$$

We recall that, under fairly general conditions, a flow can be represented by a suspension flow. Moreover, in our main example (billiard flow), this representation is natural. We study here the averaging problem for the suspension flow $\left(Y_{t}\right)_{t \geq 0}$ defined over the probabilised dynamical system $(\Omega, \nu, \sigma)$ by the measurable function $\tau: \Omega \rightarrow] 0 ;+\infty\left[\right.$ satisfying $C^{-1} \leq \tau \leq C$ for some constant $C>0$ (cf. Sect. 3.1). We suppose without any loss of generality that $\left(Y_{t}\right)_{t \geq 0}$ is given by an invertible suspension flow $\left(Y_{t}\right)_{t \in \mathbf{R}}$ defined over an invertible dynamical system $(\Omega, \nu, \sigma)$ (we can replace $(\Omega, \nu, \sigma)$ by its natural extension).

For such a flow, we weaken hypotheses on the function $f$ in a natural way. Indeed, the problem of the study of $\left(E_{t}^{\varepsilon}\right)_{\varepsilon}$ is well defined for a function $f: \mathbf{R}^{d} \times \mathcal{M} \rightarrow \mathbf{R}^{d}$ satisfying the following properties:

(a) $f$ is measurable, uniformly bounded and uniformly Lipschitz continuous in the first parameter;

(b) for any $(x, \omega) \in \mathbf{R}^{d} \times \Omega, s \mapsto f(x,(\omega, s))$ is continuous on $] 0 ; \tau(\omega)$ [ and the following limits exist: $\lim _{s \rightarrow 0+} f(x,(\omega, s))$ and $\lim _{s \rightarrow \tau(\omega)-} f(x,(\omega, s))$.

We denote by $\mathcal{L}$ the set of functions satisfying these properties. Let $f$ be in $\mathcal{L}$. Then, for any $(x, y) \in \mathbf{R}^{d} \times \mathcal{M}$ and any $\varepsilon>0$, we consider the function $t \mapsto X_{t}^{\varepsilon}(x, y)$, continuous, piecewise $C^{1}$, satisfying the differential equation (1.1.1) for any $t$ such that $Y_{\frac{t}{\varepsilon}}(y) \notin \Omega \times\{0\}$, with the initial condition $X_{0}^{\varepsilon}(x, y)=x$. With this definition, for any $x$ in $\mathbf{R}^{d}$ and any real number $t>0, X_{t}^{\varepsilon}(x, \cdot)$ is a random variable. Moreover, for any $x$ in $\mathbf{R}^{d}, f(x, \cdot)$ is measurable. So, the solution $\left(W_{t}(x)\right)_{t}$ of equation (1.1.2) with the initial condition $W_{0}(x)=x$ is also well defined.

Let $k \geq 1$ be an integer. We shall denote by $\mathcal{C}_{b}^{(k)}$ the set of functions $f: \mathbf{R}^{d} \times \mathcal{M} \rightarrow \mathbf{R}^{d}$ in $\mathcal{L}$ and in $C_{b}^{k, *}\left(\mathbf{R}^{d} \times \mathcal{M}\right)$.

We write $\bar{\tau}:=\int_{\Omega} \tau(\omega) \mathrm{d} \nu(\omega), \tilde{f}(x, y)=f(x, y)-\bar{f}(x)$. We define, for any real number $t>0$ and any $\omega \in \Omega$,

$$
n(t, \omega):=\max \left\{n \geq 0: \sum_{k=0}^{n-1} \tau\left(\sigma^{k}(\omega)\right) \leq t\right\}
$$

We define $F(x, \omega):=\int_{0}^{\tau(\omega)} f(x,(\omega, s)) \mathrm{d} s$. We notice that, if $f$ is in $\mathcal{L}$, then $\left(E_{t}^{\varepsilon}(x, \cdot)\right)_{t}$ is a continuous process and the function $F$ is well defined, measurable, uniformly bounded and uniformly Lipschitz continuous in the first parameter. Consequently, for any $x \in \mathbf{R}^{d}, F(x, \cdot)$ is measurable. Moreover, if $f$ is in $\mathcal{C}_{b}^{(2)}$, then the function $F$ is in $C_{b}^{2, *}$ and we have

$$
D_{1} F(x, \omega)=\int_{0}^{\tau(\omega)} D_{1} f(x,(\omega, s)) \mathrm{d} s \text { and } D_{1}^{2} F(x, \omega)=\int_{0}^{\tau(\omega)} D_{1}^{2} f(x,(\omega, s)) \mathrm{d} s .
$$


We shall make the following assumption:

Hypothesis 3.1.1. The function $f$ is in $\mathcal{C}_{b}^{(2)}$. The Hypothesis 2.1.2 is satisfied for $F$ and for a normed vector space $V$. Moreover, the function $\tau$ is in $V$ and satisfies $C^{-1} \leq \tau \leq C$ for some constant $C>0$.

We fix a real number $T_{0}>0$. The main result of this section is the following one:

Theorem 3.1.2. Under Hypothesis 3.1.1, for any integer $L \geq 1$, we have

$$
\sup _{0<\varepsilon<1} \sup _{x \in \mathbf{R}^{d}} \sup _{0 \leq t \leq T_{0}}\left\|\frac{E_{t}^{\varepsilon}(x, \cdot)}{\sqrt{\varepsilon}}\right\|_{L}<+\infty
$$

Moreover, the process $\left(\frac{E_{t}^{\varepsilon}(x, \cdot)}{\sqrt{\varepsilon}}\right)_{0 \leq t \leq T_{0}}$ converges in distribution, for the probability measure $\mu$ and for the uniform topology on $C\left(\left[0, T_{0}\right]\right)$ (when $\varepsilon$ goes to 0$)$, to the process $\left(E_{t}^{0}(x, \cdot)\right)_{0 \leq t \leq T_{0}}$ solution of:

$$
E_{t}^{0}(x, \cdot)=\tilde{V}_{t}^{0}(x, \cdot)+\int_{0}^{t} D \bar{f}\left(w_{s}(x)\right) \cdot E_{s}^{0}(x, \cdot) \mathrm{d} s,
$$

with the initial condition $E_{0}^{0}(x, \cdot)=0$ and where $\tilde{V}^{0}(x, \cdot)$ is a continuous Gaussian process with independent increments, centered and such that we have:

$$
\operatorname{Cov}\left(\tilde{V}_{t}^{0}(x, \cdot)\right)=\left(\int_{0}^{t} B_{i, j}\left(w_{s}(x)\right) \mathrm{d} s\right)_{i, j},
$$

with $B_{i, j}(x):=\lim _{t \rightarrow+\infty} \frac{1}{t} \int_{0}^{t} \int_{0}^{t} \mathbf{E}_{\mu}\left[\tilde{f}_{i}\left(x, Y_{s}(\cdot)\right) \cdot \tilde{f}_{j}\left(x, Y_{u}(\cdot)\right)\right] \mathrm{d} s \mathrm{~d} u$.

The remainder of this section is devoted to the proof of this theorem.

\subsection{Discretisation of the model}

Following [11], we get the following result, that leads us to the study of the averaging problem in terms of a dynamical system given by a transformation.

Hypothesis 3.2.1. The function $\tau$ satisfies $C^{-1} \leq \tau \leq C$ for some constant $C>0$ and the function $f$ : $\mathbf{R}^{d} \times \mathcal{M} \rightarrow \mathbf{R}^{d}$ is in $\mathcal{L}$.

We consider the solutions $\left(x_{t}^{\varepsilon}(x, \cdot)\right)_{t}$ and $\left(w_{t}(x)\right)_{t}$ of equations $(1.1 .3)$ with the initial conditions $x_{0}^{\varepsilon}(x, \cdot)=$ $w_{0}(x)=x$ for $F(x, \omega):=\int_{0}^{\tau(\omega)} f(x,(\omega, s)) \mathrm{d} s$. We observe that, since $\bar{F}=\bar{\tau} \bar{f}$, we have $w_{t}(x)=W_{\bar{\tau} t}(x)$.

Proposition 3.2.2 (Kifer). Under Hypothesis 3.2.1, there exists a real number $C_{0}>0$ such that, for any $\varepsilon>0$, we have

$$
\sup _{x \in \mathbf{R}^{d}} \sup _{t \in\left[0, T_{0}\right]} \sup _{\omega \in \Omega} \sup _{0 \leq s \leq \tau(\omega)}\left|X_{t}^{\varepsilon}(x,(\omega, s))-x_{\varepsilon n\left(\frac{t}{\varepsilon}, \omega\right)}^{\varepsilon}(x, \omega)\right| \leq C_{0} \varepsilon .
$$

We consider the function $\tilde{w}_{t}^{\varepsilon}(x, \omega)$ given by

$$
\frac{\mathrm{d} \tilde{w}_{t}^{\varepsilon}(x, \omega)}{\mathrm{d} t}=\tau\left(\sigma^{\left\lfloor\frac{t}{\varepsilon}\right\rfloor}(\omega)\right) \bar{f}\left(\tilde{w}_{t}^{\varepsilon}(x, \omega)\right)
$$

with the initial condition $\tilde{w}_{0}^{\varepsilon}(x, \omega)=x$. We notice that, according to Proposition 3.2.2, we have: $\sup _{x \in \mathbf{R}^{d}} \sup _{0 \leq t \leq T_{0}} \sup _{\omega \in \Omega}\left|W_{t}(x)-\tilde{w}_{\varepsilon n\left(\frac{t}{\varepsilon}, \omega\right)}^{\varepsilon}(x, \omega)\right|=O(\varepsilon)$. Thus, we have

$$
\frac{E_{t}^{\varepsilon}(x,(\omega, s))}{\sqrt{\varepsilon}}=\frac{e_{\varepsilon n\left(\frac{t}{\varepsilon}, \omega\right)}^{\varepsilon}(x, \omega)}{\sqrt{\varepsilon}}-\frac{f_{\varepsilon n\left(\frac{t}{\varepsilon}, \omega\right)}^{\varepsilon}(x, \omega)}{\sqrt{\varepsilon}}+O(\sqrt{\varepsilon}),
$$


where we have defined $f_{t}(x, \omega):=\tilde{w}_{t}^{\varepsilon}(x, \omega)-w_{t}(x)$. We observe that $f_{t}^{\varepsilon}(x, \omega)$ is obtained as $e_{t}^{\varepsilon}(x, \omega)$ by considering the function $K(x, \omega):=\tau(\omega) \bar{f}(x)$ instead of the function $F(x, \omega)$. According to the following result, results of convergence in distribution established in $(\Omega, \nu)$ are still true in $(\mathcal{M}, \mu)$.

Proposition 3.2.3. Let us suppose that the sequence of random variables $\left(\frac{1}{n} \sum_{k=0}^{n-1} \tau \circ \sigma^{k}\right)_{n}$ converges in probability (for $\nu$ ) to $\bar{\tau}$. Let $t>0$ be a real number and $x$ be a point in $\mathbf{R}^{d}$. If $\left(\frac{e_{t}^{\varepsilon}(x, \cdot)}{\sqrt{\varepsilon}}\right)_{\varepsilon>0}$ converges in distribution $($ for $\nu$ ) to a random variable $Z$ (when $\varepsilon$ goes to 0$)$, then the family of random variables $\left(G_{\varepsilon}:(\omega, s) \mapsto \frac{e_{t}^{\varepsilon}(x, \omega)}{\sqrt{\varepsilon}}\right)$ $)_{\varepsilon>0}$ converges in distribution (for $\mu$ ) to the random variable $Z$ (when $\varepsilon$ goes to 0 ).

Proof. Let $z \in \mathbf{R}^{d}$ be fixed. We have $\mathbf{E}_{\mu}\left[\mathrm{e}^{i\left\langle z, G_{\varepsilon}(\cdot)\right\rangle}\right]=\frac{1}{\bar{\tau}} \int_{\Omega} \tau(\omega) \mathrm{e}^{i\left\langle z, G_{\varepsilon}(\omega, 0)\right\rangle} \mathrm{d} \nu(\omega)$ and, for any integer $m \geq 1$,

$$
\left|\frac{1}{\bar{\tau}} \int_{\Omega} \tau(\omega) \mathrm{e}^{i\left\langle z, G_{\varepsilon}(\omega, 0)\right\rangle} \mathrm{d} \nu(\omega)-\int_{\Omega} \mathrm{e}^{i\left\langle z, G_{\varepsilon}(\omega, 0)\right\rangle} \mathrm{d} \nu(\omega)\right| \leq A_{m}^{\varepsilon}+B_{m}^{\varepsilon}
$$

with

and

$$
A_{m}^{\varepsilon}:=\frac{1}{\bar{\tau}} \frac{1}{m} \sum_{k=0}^{m-1}\left|\int_{\Omega} \tau \circ \sigma^{k}(\omega)\left(\mathrm{e}^{i\left\langle z, G_{\varepsilon}\left(\sigma^{k}(\omega), 0\right)\right\rangle}-\mathrm{e}^{i\left\langle z, G_{\varepsilon}(\omega, 0)\right\rangle}\right) \mathrm{d} \nu(\omega)\right|
$$

$$
B_{m}^{\varepsilon}:=\frac{1}{\bar{\tau}}\left|\int_{\Omega} \frac{1}{m} \sum_{k=0}^{m-1}\left(\tau \circ \sigma^{k}(\omega)-\bar{\tau}\right) \mathrm{e}^{i\left\langle z, G_{\varepsilon}(\omega, 0)\right\rangle} \mathrm{d} \nu(\omega)\right|
$$

Let $\alpha>0$ be a real number. We fix an integer $m \geq 1$ such that we have $\nu\left(\frac{1}{m}\left|\sum_{k=0}^{m-1}\left(\tau \circ \sigma^{k}-\bar{\tau}\right)\right| \geq \alpha\right) \leq \alpha$. We get $B_{m}^{\varepsilon} \leq \frac{\alpha}{\bar{\tau}}+\frac{\max \tau}{\bar{\tau}} \alpha$. On the other hand, we have

$$
A_{m}^{\varepsilon} \leq \mathrm{d} \frac{\sup _{\Omega} \tau}{\bar{\tau}} \frac{1}{m} \sum_{k=0}^{m-1} \int_{\Omega}|z| .\left|G_{\varepsilon}\left(\sigma^{k}(\omega), 0\right)-G_{\varepsilon}(\omega, 0)\right| \mathrm{d} \nu(\omega) .
$$

Now, for any $\omega \in \Omega$, any integer $k=0, \ldots, m-1$ and any real number $\varepsilon>0$ such that $\varepsilon m \leq t$, we have

$$
\begin{aligned}
\left|G_{\varepsilon}\left(\sigma^{k}(\omega), 0\right)-G_{\varepsilon}(\omega, 0)\right| & =\left|\frac{x_{t}^{\varepsilon}\left(x, \sigma^{k}(\omega)\right)-x_{t}^{\varepsilon}(x, \omega)}{\sqrt{\varepsilon}}\right| \\
& =\frac{1}{\sqrt{\varepsilon}}\left|\int_{0}^{t} F\left(x_{s}^{\varepsilon}\left(x, \sigma^{k}(\omega)\right), \sigma^{\left\lfloor\frac{s+\varepsilon k}{\varepsilon}\right\rfloor}(\omega)\right)-F\left(x_{s}^{\varepsilon}(x, \omega), \sigma^{\left\lfloor\frac{s}{\varepsilon}\right\rfloor}(\omega)\right), \mathrm{d} s\right| \\
& \left.\leq 2 \sqrt{\varepsilon} k\|F\|_{\infty}+\frac{1}{\sqrt{\varepsilon}} \int_{0}^{t} L_{F} \mid x_{s}^{\varepsilon}\left(x, \sigma^{k}(\omega)\right)-x_{s+\varepsilon k}^{\varepsilon}(x, \omega)\right) \mid \mathrm{d} s \\
& \leq\left(2+t L_{F}\right) \sqrt{\varepsilon} k\|F\|_{\infty}+L_{F} \int_{0}^{t}\left|\frac{\left.x_{s}^{\varepsilon}\left(x, \sigma^{k}(\omega)\right)-x_{s}^{\varepsilon}(x, \omega)\right)}{\sqrt{\varepsilon}}\right| \mathrm{d} s .
\end{aligned}
$$

According to Gronwall lemma, we get $\left|G_{\varepsilon}\left(\sigma^{k}(\omega), 0\right)-G_{\varepsilon}(\omega, 0)\right| \leq\left(2+t L_{F}\right)\|F\|_{\infty} \sqrt{\varepsilon} m \mathrm{e}^{L_{F} t}$.

\subsection{Estimations in norm}

According to Theorem 2.1.3, we make the following remark:

Remark. Under Hypothesis 3.1.1, for any real number $T^{\prime}>0$ and any integer $L \geq 1$, we have

$$
\sup _{0<\varepsilon<1} \sup _{t \in\left[0 ; T^{\prime}\right]} \sup _{x \in \mathbf{R}^{d}} \frac{1}{\sqrt{\varepsilon}}\left\|e_{t}^{\varepsilon}(x, \cdot)\right\|_{L}<+\infty \text { and } \sup _{0<\varepsilon<1} \sup _{t \in\left[0 ; T^{\prime}\right]} \sup _{x \in \mathbf{R}^{d}} \frac{1}{\sqrt{\varepsilon}}\left\|f_{t}^{\varepsilon}(x, \cdot)\right\|_{L}<+\infty .
$$


We establish here the first conclusion of Theorem 3.1.2:

Theorem 3.3.1. Under Hypothesis 3.1.1, for any integer $L \geq 1$, we have

$$
\sup _{0<\varepsilon<1} \sup _{0 \leq t \leq T_{0}} \sup _{x \in \mathbf{R}^{d}}\left\|\frac{E_{t}(x, \cdot)}{\sqrt{\varepsilon}}\right\|_{L}<+\infty .
$$

According to Proposition 3.2.2 and to the previous remark, this is a consequence of the following result:

Proposition 3.3.2. Under Hypothesis 3.1.1, for any real number $T^{\prime}>0$ and any integer $L \geq 2$, we have

$$
\lim _{\varepsilon \rightarrow 0} \sup _{0 \leq t \leq T^{\prime}} \sup _{x \in \mathbf{R}^{d}} \varepsilon^{-\frac{1}{2}}\left\|e_{\varepsilon n\left(\frac{t}{\varepsilon}, \cdot\right)}^{\varepsilon}(x, \cdot)-e_{\frac{t}{\bar{\tau}}}^{\varepsilon}(x, \cdot)\right\|_{2 L}=0
$$

and

$$
\lim _{\varepsilon \rightarrow 0} \sup _{0 \leq t \leq T^{\prime}} \sup _{x \in \mathbf{R}^{d}} \varepsilon^{-\frac{1}{2}}\left\|f_{\varepsilon n\left(\frac{t}{\varepsilon}, \cdot\right)}^{\varepsilon}(x, \cdot)-f_{\frac{t}{\tau}}^{\varepsilon}(x, \cdot)\right\|_{2 L}=0
$$

We can prove Proposition 3.3.2 using the same arguments as [11] with the help of the following lemma:

Lemma 3.3.3. Let $L \geq 1$ be an integer. There exists a real number $C_{L}>0$ such that we have

$$
\sup _{N \geq 1}\left\|\frac{1}{\sqrt{N}} \sum_{k=0}^{N-1}\left(\tau \circ \sigma^{k}-\bar{\tau}\right)\right\|_{2 L}^{2 L} \leq C_{L}<+\infty
$$

and there exists a constant $K_{L}>0$ such that, for any real numbers $\varepsilon>0, K>2 \bar{\tau} \varepsilon$ and $t>0$, we have

$$
\nu\left(\left\{\left|\bar{\tau} \varepsilon n\left(\frac{t}{\varepsilon}, \cdot\right)-t\right| \geq K\right\}\right) \leq K_{L} t^{2 L} \varepsilon^{2 L} K^{-4 L} .
$$


Proof. The first result is a consequence of the fact that $\tau$ is in $V$ and of Lemma 2.3.4. Let a real number $K$ such that $K \geq 2 \bar{\tau} \varepsilon$. We have

$$
\begin{aligned}
& \nu\left(\left\{\left|\bar{\tau} \varepsilon n\left(\frac{t}{\varepsilon}, \cdot\right)-t\right| \geq K\right\}\right) \leq \nu\left(\left\{\bar{\tau} \varepsilon n\left(\frac{t}{\varepsilon}, \cdot\right)-t \geq K\right\}\right)+\nu\left(\left\{\bar{\tau} \varepsilon n\left(\frac{t}{\varepsilon}, \cdot\right)-t \leq-K\right\}\right) \\
& \leq \nu\left(\left\{n\left(\frac{t}{\varepsilon}, \cdot\right) \geq \frac{t+K}{\bar{\tau} \varepsilon}\right\}\right)+\nu\left(\left\{n\left(\frac{t}{\varepsilon}, \cdot\right) \leq \frac{t-K}{\bar{\tau} \varepsilon}\right\}\right) \\
& \leq \nu\left(\left\{\sum_{k=0}^{\left\lceil\frac{t+K}{\varepsilon \bar{\tau}}\right\rceil-1} \tau \circ \sigma^{k} \leq \frac{t}{\varepsilon}\right\}\right)+\nu\left(\left\{\sum_{k=0}^{\left\lfloor\frac{t-K}{\varepsilon \bar{\tau}}\right\rfloor} \tau \circ \sigma^{k} \geq \frac{t}{\varepsilon}\right\}\right) \\
& \leq \nu\left(\left\{\sum_{k=0}^{\left\lceil\frac{t+K}{\varepsilon \bar{\tau}}\right\rceil-1}\left(\tau \circ \sigma^{k}-\bar{\tau}\right) \leq \frac{t}{\varepsilon}-\bar{\tau}\left(\frac{t+K}{\varepsilon \bar{\tau}}\right)\right\}\right) \\
& +\nu\left(\left\{\sum_{k=0}^{\left\lfloor\frac{t-K}{\varepsilon \bar{\tau}}\right\rfloor}\left(\tau \circ \sigma^{k}-\bar{\tau}\right) \geq \frac{t}{\varepsilon}-\bar{\tau}\left(\frac{t-K}{\varepsilon \bar{\tau}}+1\right)\right\}\right) \\
& \leq \nu\left(\left\{\sum_{k=0}^{\left\lceil\frac{t+K}{\varepsilon \bar{\tau}}\right\rceil-1}\left(\tau \circ \sigma^{k}-\bar{\tau}\right) \leq-\frac{K}{\varepsilon}\right\}\right)+\nu\left(\left\{\sum_{k=0}^{\left\lfloor\frac{t-K}{\varepsilon \bar{\tau}}\right\rfloor}\left(\tau \circ \sigma^{k}-\bar{\tau}\right) \geq \frac{K}{\varepsilon}-\bar{\tau}\right\}\right) \\
& \leq \frac{\left\|\sum_{k=0}^{\left\lceil\frac{t+K}{\varepsilon \tau}\right\rceil-1}\left(\tau \circ \sigma^{k}-\bar{\tau}\right)\right\|_{4 L}^{4 L}}{\varepsilon^{-4 L} K^{4 L}}+\frac{\left\|\sum_{k=0}^{\left\lfloor\frac{t-K}{\varepsilon \tau}\right\rfloor}\left(\tau \circ \sigma^{k}-\bar{\tau}\right)\right\|_{4 L}^{4 L}}{\varepsilon^{-4 L}(K-\bar{\tau} \varepsilon)^{4 L}}
\end{aligned}
$$

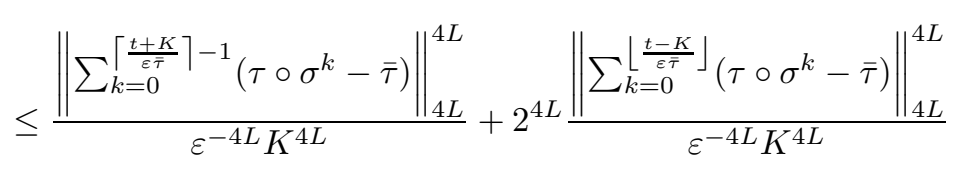

$$
\begin{aligned}
& \leq 2 \sup _{n \geq 1}\left\|\frac{1}{\sqrt{n}} \sum_{k=0}^{n-1}\left(\tau \circ \sigma^{k}-\bar{\tau}\right)\right\|_{4 L}^{4 L} 2^{4 L} \frac{\left(\frac{t+K}{\varepsilon \bar{\tau}}+1\right)^{2 L}}{\varepsilon^{-4 L} K^{4 L}}
\end{aligned}
$$

If $t \geq K$, then we have $\frac{t+K}{\varepsilon \bar{\tau}}+1=\frac{1}{\varepsilon \bar{\tau}}(t+K+\varepsilon \bar{\tau}) \leq \frac{1}{\varepsilon \bar{\tau}} \frac{5}{2} t$ and therefore $\nu\left(\left\{\left|\bar{\tau} \varepsilon n\left(\frac{t}{\varepsilon}, \cdot\right)-t\right| \geq K\right\}\right) \leq$ $2^{1+2 L} \cdot 5^{2 L} C \frac{t^{2 L} \varepsilon^{2 L} K^{-4 L}}{\bar{\tau}^{2 L}}$.

If $t<\varepsilon \min \tau<K$, then we have $n\left(\frac{t}{\varepsilon}, \cdot\right)=0$ and therefore $\nu\left(\left\{\left|\bar{\tau} \varepsilon n\left(\frac{t}{\varepsilon}, \cdot\right)-t\right| \geq K\right\}\right)=0 . \varepsilon^{2 L} t^{2 L} K^{-4 L}$. 
If $\varepsilon \min \tau \leq t<K$, then we have

$$
\begin{aligned}
\nu\left(\left\{\left|\bar{\tau} \varepsilon n\left(\frac{t}{\varepsilon}, \cdot\right)-t\right| \geq K\right\}\right) & \leq \nu\left(\left\{\bar{\tau} \varepsilon n\left(\frac{t}{\varepsilon}, \cdot\right)-t \geq K\right\}\right) \\
& \leq \frac{\left\|\sum_{k=0}^{\left\lceil\frac{t+K}{\varepsilon \bar{\tau}}-1\right.}\left(\tau \circ \sigma^{k}-\bar{\tau}\right)\right\|_{8 L}^{8 L}}{\varepsilon^{-8 L} K^{8 L}} \\
& \leq \sup _{n \geq 1}\left\|\frac{1}{\sqrt{n}} \sum_{k=0}^{n-1}\left(\tau \circ \sigma^{k}-\bar{\tau}\right)\right\|_{8 L}^{8 L} \frac{\left(\frac{t+K}{\varepsilon \bar{\tau}}+1\right)^{4 L}}{\varepsilon^{-8 L} K^{8 L}} \\
& \leq\left(\frac{5}{2}\right)^{4 L} \sup _{n \geq 1}\left\|\frac{1}{\sqrt{n}} \sum_{k=0}^{n-1}\left(\tau \circ \sigma^{k}-\bar{\tau}\right)\right\|_{8 L}^{8 L} \frac{\left(\frac{K}{\varepsilon \bar{\tau}}\right)^{4 L}}{\varepsilon^{-8 L} K^{8 L}} \\
& \leq\left(\frac{5}{2}\right)^{4 L} \sup _{n \geq 1}\left\|\frac{1}{\sqrt{n}} \sum_{k=0}^{n-1}\left(\tau \circ \sigma^{k}-\bar{\tau}\right)\right\|_{8 L}^{8 L} \frac{\varepsilon^{4 L} K^{-4 L}}{\bar{\tau}^{4 L}} \\
& \leq\left(\frac{5}{2}\right)^{4 L} \sup _{n \geq 1}^{4 L}\left\|\frac{1}{\sqrt{n}} \sum_{k=0}^{n-1}\left(\tau \circ \sigma^{k}-\bar{\tau}\right)\right\|_{8 L}^{8 L} \frac{\varepsilon^{2 L} t^{2 L} K^{-4 L}}{(\min \tau)^{2 L} \bar{\tau}^{4 L}} .
\end{aligned}
$$

Sketch of the proof of Proposition 3.3.2. We only give the ideas of the proof which follows from [11]. We are interested in the proof of (3.3.1); the proof of (3.3.2) being obtained in the same way with $\bar{f}$ instead of $f$ (and then $\tau(\omega) \bar{f}(x)$ instead of $F(x, \omega))$. For any real number $t$ in $\left[0 ; T_{0}\right]$, we have

$$
\begin{aligned}
\frac{1}{\sqrt{\varepsilon}}\left\|e_{\varepsilon n\left(\frac{t}{\varepsilon}, \cdot\right)}^{\varepsilon}(x, \cdot)-e_{\frac{t}{\bar{\tau}}}^{\varepsilon}(x, \cdot)\right\|_{2 L} & =\frac{1}{\sqrt{\varepsilon}}\left\|\int_{\frac{t}{\bar{\tau}}}^{\varepsilon n\left(\frac{t}{\varepsilon}, \cdot\right)}\left(F\left(x_{s}^{\varepsilon}(x, \cdot), \sigma^{\left\lfloor\frac{s}{\varepsilon}\right\rfloor}(\cdot)\right)-\bar{F}\left(w_{s}(x)\right)\right) \mathrm{d} s\right\|_{2 L} \\
& \leq\left\|I_{t}^{\varepsilon}(x, \cdot)\right\|_{2 L}+\left\|J_{t}^{\varepsilon}(x, \cdot)\right\|_{2 L},
\end{aligned}
$$

with

and

$$
I_{t}^{\varepsilon}(x, \cdot):=\frac{1}{\sqrt{\varepsilon}} \int_{\frac{t}{\bar{\tau}}}^{\varepsilon n\left(\frac{t}{\varepsilon}, \cdot\right)} \tilde{F}\left(w_{s}(x, \cdot), \sigma^{\left\lfloor\frac{s}{\varepsilon}\right\rfloor}(\cdot)\right) \mathrm{d} s=v_{\varepsilon n\left(\frac{t}{\varepsilon}, \cdot\right)}^{\varepsilon}(x, \cdot)-v_{\frac{t}{\varepsilon}}^{\varepsilon}(x, \cdot)
$$

$$
J_{t}^{\varepsilon}(x, \cdot):=L_{F} \int_{\frac{t}{\bar{\tau}}}^{\varepsilon n\left(\frac{t}{\varepsilon}, \cdot\right)}\left|\frac{e_{s}^{\varepsilon}(x, \cdot)}{\sqrt{\varepsilon}}\right| \mathrm{d} s,
$$

where $L_{F}$ is the Lipschitz coefficient of $F$, for the regularity in the first variable. Let $\alpha$ be a real number such that $\frac{1}{2 L}<\alpha<\frac{1}{2}$. According to the previous lemma, via calculations we omit here and which follow from [11], we get: $\left\|I_{t}^{\varepsilon}(x, \cdot)\right\|_{2 L} \leq C^{t e}\left(\varepsilon^{\alpha}+\varepsilon^{\frac{\alpha}{2}-\frac{1}{4 L}}+\varepsilon^{\frac{3}{2}-3 \alpha}\right)$ and $\left\|J_{t}^{\varepsilon}(x, \cdot)\right\|_{2 L} \leq C^{t e}\left(\varepsilon^{\alpha}+\varepsilon^{\frac{3}{2}-3 \alpha}\right)$.

According to the foregoing, Proposition 3.3.2 gives us the results of convergence of Theorem 3.1.2 in the sense of finite distributions. But, to prove the convergence in distribution, we need an additional argument $(c f$. Sect. 3.4).

\subsection{Convergence in distribution}

We complete the proof of Theorem 3.1.2 which is given in several steps. Proposition 3.2.2 leads us to the study of the asymptotical behavior of $\left(\left(e_{\varepsilon n\left(\frac{t}{\varepsilon}, \cdot\right)}^{\varepsilon}(x, \cdot)-f_{\varepsilon n\left(\frac{t}{\varepsilon}, \cdot\right)}^{\varepsilon}(x, \cdot)\right)_{0 \leq t \leq T_{0}}\right)_{\varepsilon>0}$ when $\varepsilon$ goes to 0 . First, we prove the following lemma: 
Lemma 3.4.1. Let a real number $T^{\prime}>0$ and a point $x$ in $\mathbf{R}^{d}$ be fixed. The family of processes $\left(\left(\frac{e_{t}^{\varepsilon}(x, \cdot)-f_{t}^{\varepsilon}(x, \cdot)}{\sqrt{\varepsilon}}\right)_{0 \leq t \leq T^{\prime}}\right)_{\varepsilon>0}$ converges in distribution (for $\nu$ and for the uniform topology on $C\left(\left[0, T^{\prime}\right]\right)$ when $\varepsilon$ goes to 0$)$ to the process $\tilde{y}^{0}(x, \cdot)$ solution of:

$$
\tilde{y}_{t}^{0}(x, \cdot)=\tilde{v}_{t}^{0}(x, \cdot)+\int_{0}^{t} D \bar{F}\left(w_{s}(x)\right) \cdot \tilde{y}_{s}^{0}(x, \cdot) \mathrm{d} s
$$

where $\tilde{v}^{0}(x, \cdot)$ is a continuous Gaussian process with independent increments, centered and such that $\operatorname{Cov}\left(\tilde{v}_{t}^{0}(x, \cdot)\right)=$ $\left(\int_{0}^{t} A_{i, j}\left(w_{s}(x)\right) \mathrm{d} s\right)_{i, j}$, with

$$
A_{i, j}(x):=\lim _{t \rightarrow+\infty} \frac{1}{t} \int_{0}^{t} \int_{0}^{t} \mathbf{E}_{\nu}\left[G_{i}\left(x, \sigma^{\lfloor s\rfloor}(\cdot)\right) \cdot G_{j}\left(x, \sigma^{\lfloor u\rfloor}(\cdot)\right)\right] \mathrm{d} s \mathrm{~d} u,
$$

and $G(x, \omega):=F(x, \omega)-\tau(\omega) \bar{f}(x)=\int_{0}^{\tau(\omega)} \tilde{f}(x,(\omega, u)) \mathrm{d} u$

Proof. We can prove that the family of processes $\left(\left(\frac{e_{t}^{\varepsilon}(x, \cdot)-f_{t}^{\varepsilon}(x, \cdot)}{\sqrt{\varepsilon}}\right)_{0 \leq t \leq T^{\prime}}\right)_{\varepsilon>0}$ is tight (for $\left.\nu\right)$ as we proved the tightness of the family of processes $\left(\left(\frac{e_{t}^{\varepsilon}(x, \cdot)}{\sqrt{\varepsilon}}\right)_{0 \leq t \leq T^{\prime}}\right)_{\varepsilon>0}$ in Section 2.4. Moreover, we have seen, in the proof of Theorem 2.1.3 (Sect. 2.4), that we have $\lim _{\varepsilon \rightarrow 0} \sup _{0 \leq t \leq T^{\prime}}\left\|\frac{e_{t}^{\varepsilon}(x, \cdot)}{\sqrt{\varepsilon}}-y_{t}^{\varepsilon}(x, \cdot)\right\|_{1}=0$. Taking $\bar{f}$ instead of $f, H(x, \omega):=\tau(\omega) \bar{f}(x)$ instead of $F$ and $\tilde{H}:=H-\bar{\tau} \cdot \bar{f}$ instead of $\tilde{F}$, we get, in the same way, $\lim _{\varepsilon \rightarrow 0} \sup _{0 \leq t \leq T^{\prime}}\left\|\frac{f_{t}^{\varepsilon}(x, \cdot)}{\sqrt{\varepsilon}}-\check{y}_{s}^{\varepsilon}(x, \cdot)\right\|_{1}=0$, with $\check{y}_{t}^{\varepsilon}(x, \cdot)=\check{v}_{t}^{\varepsilon}(x, \cdot)+\int_{0}^{t} D \bar{F}\left(w_{s}(x)\right) \cdot \check{y}_{s}^{\varepsilon}(x, \cdot) \mathrm{d} s$ and $\check{v}_{t}^{\varepsilon}(x, \cdot):=$ $\frac{1}{\sqrt{\varepsilon}} \int_{0}^{t} \tilde{H}\left(w_{u}(x), \sigma^{\left\lfloor\frac{u}{\varepsilon}\right\rfloor}(\omega)\right) \mathrm{d} u=\frac{1}{\sqrt{\varepsilon}} \int_{0}^{t}\left(\tau\left(\sigma^{\left\lfloor\frac{u}{\varepsilon}\right\rfloor}(\omega)\right)-\bar{\tau}\right) \bar{f}\left(w_{u}(x)\right) \mathrm{d} u$. This leads us to the study of $\tilde{y}_{t}^{\varepsilon}(x, \cdot):=$ $y_{t}^{\varepsilon}(x, \cdot)-\check{y}_{t}^{\varepsilon}(x, \cdot)$. Let us define:

$$
\begin{aligned}
\tilde{v}_{t}^{\varepsilon}(x, \cdot) & :=v_{t}^{\varepsilon}(x, \cdot)-\check{v}_{t}^{\varepsilon}(x, \cdot) \\
& =\frac{1}{\sqrt{\varepsilon}} \int_{0}^{t} F\left(w_{u}(x), \sigma^{\left\lfloor\frac{u}{\varepsilon}\right\rfloor}(\omega)\right)-\tau\left(\sigma^{\left\lfloor\frac{u}{\varepsilon}\right\rfloor}(\omega)\right) \bar{f}\left(w_{u}(x)\right) \mathrm{d} u \\
& =\frac{1}{\sqrt{\varepsilon}} \int_{0}^{t} G\left(w_{u}(x), \sigma^{\left\lfloor\frac{u}{\varepsilon}\right\rfloor}(\omega)\right) \mathrm{d} u .
\end{aligned}
$$

Moreover, we have: $\tilde{y}_{t}^{\varepsilon}(x, \cdot)=\tilde{v}_{t}^{\varepsilon}(x, \cdot)+\int_{0}^{t} D \bar{F}\left(w_{s}(x)\right) \cdot \tilde{y}_{s}^{\varepsilon}(x, \cdot) \mathrm{d} s$. Now, according to Theorem 2.3 .2 for $G(x, \omega)=$ $F(x, \omega)-\tau(\omega) \bar{f}(x)$, the family of processes $\left(\left(\tilde{v}^{\varepsilon}(x, \cdot)\right)_{0 \leq t \leq T^{\prime}}\right)_{\varepsilon>0}$ converges in distribution for $\nu$ and for the $C\left(\left[0, T^{\prime}\right]\right)$ topology (when $\varepsilon$ goes to 0 ) to a continuous Gaussian process with independent increments $\tilde{v}^{0}(x, \cdot)$ centered such that $\operatorname{Cov}\left(\tilde{v}_{t}^{0}(x, \cdot)\right)=\left(\int_{0}^{t} A_{i, j}\left(w_{s}(x)\right) \mathrm{d} s\right)_{i, j}$, with $A_{i, j}(x)$ as in the statement of the lemma. As in the proof of Proposition 2.4.1, we can show that $\left(\left(\tilde{y}^{\varepsilon}(x, \cdot)\right)_{0 \leq t \leq T^{\prime}}\right)_{\varepsilon>0}$ converges in distribution for $\nu$ (when $\varepsilon$ goes to 0$)$ to the process $\left(\tilde{y}_{t}^{0}(x, \cdot)\right)_{0 \leq t \leq T^{\prime}}$.

Lemma 3.4.2. Let $T^{\prime}>0$ be a real number and $x$ be a point in $\mathbf{R}^{d}$. The family of (continuous) processes $\left((\omega, s) \mapsto\left(\frac{e_{t}^{\varepsilon}(x, \omega)-f_{t}^{\varepsilon}(x, \omega)}{\sqrt{\varepsilon}}\right)_{0 \leq t \leq T^{\prime}}\right)_{\varepsilon>0}$ converges in distribution for $\mu$ and for the uniform topology on $C\left(\left[0, T^{\prime}\right]\right)$ (when $\varepsilon$ goes to 0$)$ to $\left(\tilde{y}_{t}^{0}(x, \cdot)\right)_{0 \leq t \leq T^{\prime}}$.

Proof. This family of processes is tight (for $\mu$ ). Moreover, we can show its convergence in the sense of finite distributions (for $\mu$ ) to $\tilde{y}^{0}(x, \cdot)$, as we proved Proposition 3.2.3. 
Using Theorem 2.1.3 for $F(x, \omega)=\tau(\omega)$ and a classical argument, we get the following lemma:

Lemma 3.4.3. The family of processes $\left(\left(\varepsilon n\left(\frac{t}{\varepsilon}, \cdot\right)\right)_{0 \leq t \leq T_{0}}\right)_{\varepsilon>0}$ converges in uniform probability (for $\left.\nu\right)$ to the deterministic process $\left(\frac{t}{\bar{\tau}}\right)_{0 \leq t \leq T_{0}}$ (when $\varepsilon$ goes to 0$)$, i.e. the random variable $\sup _{0 \leq t \leq T_{0}}\left|\varepsilon n\left(\frac{t}{\varepsilon}, \cdot\right)-\frac{t}{\bar{\tau}}\right|$ converges in probability (for $\nu$ ) to 0 (when $\varepsilon$ goes to 0$)$.

Lemma 3.4.4. Under Hypothesis 3.1.1, the family of processes $\left(\left(\frac{E_{t}^{\varepsilon}(x, \cdot)}{\sqrt{\varepsilon}}\right)_{0 \leq t \leq T_{0}}\right)_{\varepsilon>0}$ converges in distribution for $\mu$ and for the uniform topology on $C\left(\left[0, T_{0}\right]\right)$ (when $\varepsilon$ goes to 0$)$ to the process $\left(\tilde{y}_{\frac{t}{\bar{\tau}}}^{0}(x, \cdot)\right)_{0 \leq t \leq T_{0}}$.

Proof. We show that $\left(\left(\frac{e_{\varepsilon n\left(\frac{t}{\varepsilon}, \cdot\right)}^{\varepsilon}(x, \cdot)-f_{\varepsilon n\left(\frac{t}{\varepsilon}, \cdot\right)}^{\varepsilon}(x, \cdot)}{\sqrt{\varepsilon}}\right)_{0 \leq t \leq T_{0}}\right)_{\varepsilon>0}$ converges in distribution (for $\mu$ and for the uniform topology on $\left.C\left(\left[0, T_{0}\right]\right)\right)$ to the process $\left(\tilde{y}_{\frac{t}{\bar{\tau}}}^{0}(x, \cdot)\right)_{0 \leq t \leq T_{0}}$ (when $\varepsilon$ goes to 0$)$. According to Lemma 3.4.3, for any real number $T^{\prime}>0$, the family of processes $\left((\omega, s) \mapsto\left(\varepsilon n\left(\frac{t}{\varepsilon}, \omega\right)\right)_{0 \leq t \leq T^{\prime}}\right)_{\varepsilon>0}$ converges in uniform probability (for $\mu$ to the deterministic process $\left(\frac{t}{\bar{\tau}}\right)_{0 \leq t \leq T^{\prime}}$ So, family of processes $\left(\left(N(\varepsilon, t):=\varepsilon\left(n\left(\frac{t}{\varepsilon}, \cdot\right)+\frac{\frac{t}{\varepsilon}-\sum_{k=0}^{n\left(\frac{t}{\varepsilon}, \cdot\right)-1} \tau \circ \sigma^{k}(\cdot)}{\tau \circ \sigma^{n\left(\frac{t}{\varepsilon}, \cdot\right)}(\cdot)}\right)\right)_{0 \leq t \leq T^{\prime}}\right)_{\varepsilon>0}$ converges in uniform probability (for $\left.\mu\right)$ to the deterministic process $\left(\frac{t}{\bar{\tau}}\right)_{t}$ (when $\varepsilon$ goes to 0 ). So, according to Theorem 4.4 in [2], the family of processes $\left(\left(\frac{e_{t}^{\varepsilon}(x, \cdot)-f_{t}^{\varepsilon}(x, \cdot)}{\sqrt{\varepsilon}}, N(\varepsilon, t)\right)_{0 \leq t \leq \frac{T_{0}}{\min \tau}+1}\right)_{\varepsilon>0}$ converges in distribution for $\mu$ to $\left(\tilde{y}_{t}^{0}(x, \cdot), \frac{t}{\bar{\tau}}\right)_{t}$. Since $h$ : $C\left(\left[0 ; \frac{T_{0}}{\min \tau}+1\right]\right) \times C\left(\left[0 ; T_{0}\right] \rightarrow\left[0 ; \frac{T_{0}}{\min \tau}+1\right]\right) \stackrel{\longrightarrow}{\longrightarrow}\left(\left[0 ; T_{0}\right]\right)$ defined by $h(f, g)=f \circ g$ is continuous, the family of processes $\left(\left(\frac{e_{N(\varepsilon, t)}^{\varepsilon}(x, \cdot)-f_{N(\varepsilon, t)}^{\varepsilon}(x, \cdot)}{\sqrt{\varepsilon}}\right)_{0 \leq t \leq T_{0}}\right)_{\varepsilon>0}$ converges in distribution to the process $\left(\tilde{y}_{\frac{t}{\bar{\tau}}}^{0}(x, \cdot)\right)_{0 \leq t \leq T_{0}}$. Now, we have $\left|\frac{e_{\varepsilon n\left(\frac{t}{\varepsilon}, \cdot\right)}^{\varepsilon}(x, \cdot)-e_{N(\varepsilon, t)}^{\varepsilon}(x, \cdot)}{\sqrt{\varepsilon}}\right| \leq \frac{2\|F\|_{\infty} \varepsilon}{\sqrt{\varepsilon}}=2\|F\|_{\infty} \sqrt{\varepsilon}$ and idem for $f^{\varepsilon}$ instead of $e^{\varepsilon}$.

End of the proof of Theorem 3.1.2. We put $E_{t}^{0}:=\tilde{y}_{\frac{t}{\bar{\tau}}}^{0}$ and $\tilde{V}_{t}^{0}:=\tilde{v}_{\frac{t}{\bar{\tau}}}^{0}$. Then we have

$$
E_{t}^{0}(x, \cdot)=\tilde{V}_{t}^{0}(x, \cdot)+\frac{1}{\bar{\tau}} \int_{0}^{t} D \bar{F}\left(w_{\frac{s}{\bar{\tau}}}(x)\right) \cdot E_{s}^{0}(x, \cdot) \mathrm{d} s .
$$

On the other hand, we have $w_{\frac{s}{\bar{\tau}}}=W_{s}$ and $\bar{f}=\frac{\bar{F}}{\bar{\tau}}$. Moreover, $\left(\tilde{V}_{t}^{0}(x, \cdot)\right)_{t}$ is a continuous Gaussian process, centered and such that

$$
\operatorname{Cov}\left(\tilde{V}_{t}^{0}(x, \cdot)\right)=\left(\int_{0}^{\frac{t}{\tau}} A_{i, j}\left(w_{s}(x)\right) \mathrm{d} s\right)_{i, j}=\left(\int_{0}^{t} B_{i, j}\left(W_{u}(x)\right) \mathrm{d} u\right)_{i, j}
$$

with $B_{i, j}(x):=\frac{1}{\bar{\tau}} A_{i, j}(x)=\frac{1}{\bar{\tau}} A_{i, j}(x):=\lim _{t \rightarrow+\infty} \frac{1}{t} \sum_{k=0}^{\left\lfloor\frac{t}{\bar{\tau}}\right\rfloor-1} \sum_{l=0}^{\left\lfloor\frac{t}{\bar{\tau}}\right\rfloor-1} \mathbf{E}_{\nu}\left[G_{i}\left(x, \sigma^{k}(\cdot)\right) \cdot G_{j}\left(x, \sigma^{l}(\cdot)\right)\right]$ d $s \mathrm{~d} u$. Moreover, according to the foregoing, we have

$$
\lim _{t \rightarrow+\infty} \sup _{x \in \mathbf{R}^{d}} \frac{1}{\sqrt{t}}\left\|\sum_{k=0}^{n(t, \cdot)-1} G_{i}\left(x, \sigma^{k}(\cdot)\right)-\sum_{k=0}^{\left\lfloor\frac{t}{\bar{\tau}}\right\rfloor-1} G_{i}\left(x, \sigma^{k}(\cdot)\right)\right\|_{L^{4}(\nu)}=0
$$


(by using the control of $I_{1}^{\frac{1}{t}}(x, \cdot)$ obtained in the proof of Prop. 3.3.2 with $G_{i}(x, \cdot)$ instead of $\tilde{F}$ ). Thus, we get:

$$
B_{i, j}(x)=\lim _{t \rightarrow+\infty} \mathbf{E}_{\nu}\left[K_{i, j}(t, x, \cdot)\right]
$$

with $K_{i, j}(x, t, \cdot):=\frac{1}{t}\left(\sum_{k=0}^{n(t, \cdot)-1} G_{i}\left(x, \sigma^{k}(\cdot)\right)\right)\left(\sum_{l=0}^{n(t, \cdot)-1} G_{j}\left(x, \sigma^{l}(\cdot)\right)\right)$. We notice that we have:

$$
B_{i, j}(x)=\lim _{t \rightarrow+\infty} \int_{\mathcal{M}} K_{i, j}(x, t, \omega) \mathrm{d} \mu(\omega, s) .
$$

Indeed, for any integer $m \geq 1$, we have

$$
\int_{\mathcal{M}} K_{i, j}(x, t, \omega) \mathrm{d} \mu(\omega, s)=\frac{1}{\bar{\tau}} \mathbf{E}_{\nu}\left[\frac{1}{m} \sum_{s=0}^{m-1} \tau\left(\sigma^{s}(\cdot)\right) K_{i, j}\left(x, t, \sigma^{s}(\cdot)\right)\right]
$$

and thus:

$$
\left|\mathbf{E}_{\nu}\left[K_{i, j}(x, t, \cdot)\right]-\int_{\mathcal{M}} K_{i, j}(x, t, \omega) \mathrm{d} \mu(\omega, s)\right| \leq L_{i, j}^{\varepsilon}(x, t, m)+M_{i, j}^{\varepsilon}(x, t, m),
$$

with

$$
L_{i, j}^{\varepsilon}(x, t, m):=\frac{1}{m \cdot \bar{\tau}} \sum_{s=0}^{m-1}\left|\mathbf{E}_{\nu}\left[\tau \circ \sigma^{s} \cdot\left(K_{i, j}(x, t, \cdot)-K_{i, j}\left(x, t, \sigma^{s}(\cdot)\right)\right)\right]\right|
$$

and

$$
M_{i, j}^{\varepsilon}(x, t, m):=\frac{1}{\bar{\tau}}\left|\mathbf{E}_{\nu}\left[\frac{1}{m} \sum_{s=0}^{m-1}\left(\tau \circ \sigma^{s}-\bar{\tau}\right) \cdot K_{i, j}(x, t, \cdot)\right]\right| .
$$

Considering a real number $\alpha>0$ and choosing $m$ such that we have $\left\|\frac{1}{m} \sum_{s=0}^{m-1}\left(\tau \circ \sigma^{s}-\bar{\tau}\right)\right\|_{L^{2}(\nu)} \leq \alpha$, we get:

$$
M_{i, j}^{\varepsilon}(x, t, m) \leq \frac{\alpha}{\bar{\tau}}\left\|K_{i, j}(x, t, \cdot)\right\|_{L^{2}(\nu)} \leq \frac{C \alpha}{\bar{\tau}},
$$

with $C:=\sup _{x, i, j} \sup _{t>0}\left\|K_{i, j}(x, t, \cdot)\right\|_{2}$ and

$$
\begin{aligned}
L_{i, j}^{\varepsilon}(x, t, m) & \leq \frac{\sup _{\Omega} \tau}{m \cdot \bar{\tau}} \sum_{s=0}^{m-1}\left\|K_{i, j}(x, t, \cdot)-K_{i, j}\left(x, t, \sigma^{s}(\cdot)\right)\right\|_{L^{1}(\nu)} \\
& \leq 2 m C\|G\|_{\infty} \frac{\sup _{\Omega} \tau}{m \cdot \bar{\tau}} \sum_{s=0}^{m-1} \sqrt{\frac{\sup _{\Omega} \tau}{t \cdot \inf _{\Omega} \tau}}
\end{aligned}
$$

Since we have

$$
\sup _{t>0} \sup _{(\omega, s) \in \mathcal{M}}\left|\int_{0}^{t} \tilde{f}\left(x, Y_{u}(\omega, s)\right) \mathrm{d} u-\sum_{k=0}^{n(t, \cdot)-1} G\left(x, \sigma^{k}(\omega)\right)\right| \leq 3 \sup _{\Omega} \tau .\|\tilde{f}\|_{\infty},
$$

we get: $B_{i, j}(x)=\lim _{t \rightarrow+\infty} \frac{1}{t} \int_{0}^{t} \int_{0}^{t} \mathbf{E}_{\mu}\left[\tilde{f}_{i}(x, \cdot) \tilde{f}_{j}(x, \cdot)\right]$. 


\section{EXAmples}

We give examples of situations for which the Hypotheses 2.1.2 or 3.1.1 are satisfied. By direct calculations, we prove in [14] that our multiple decorrelation property is satisfied when the transfer operator acts in a quasicompact way on a convenient normed space $(V,\|\cdot\|)$. An other example to which our method may be applied is the subshift of finite type for Gibbs measure associated with a Hölder continuous function $\phi$, for the space $V$ of $\eta$-Hölder continuous functions ( $c f$. [14]). Thus, for these two examples we omit here, we obtain results already given in [11]. Moreover, we see that our multiple decorrelation property is strong, in the sense that it requires an exponential rate of decorrelation decay that is not required in [11]. Thus, we see that Kifer's method may be applied to subshifts of finite type in a weakly regular case, for which our hypothesis of multiple decorrelation is not satisfied $(c f .[14])$.

In this section, we study two other examples. We show how our method can be applied to the ergodic algebraic toral automorphisms, including ergodic not hyperbolic automorphisms to which the method developed in [11] does not apply (for a filtration obtained in the classical way). We finish with the example of the billiard flow in some domain $Q$ in $\mathbf{T}^{2}$.

\subsection{Ergodic toral automorphisms}

Each algebraic automorphism $\sigma=T$ of the torus $\Omega=\mathbf{T}^{n}$ preserves the normalised Lebesgue measure $\nu$ on $\mathbf{T}^{n}$. We recall that a toral automorphism $T$ is ergodic if and only if the matrix $M \in S L(n, \mathbf{R})$ associated to $T$ does not have any eigenvalue root of the unity and that a toral automorphism $T$ is said to be hyperbolic if the matrix $M$ does not have any eigenvalue of modulus 1 . So, each hyperbolic toral algebraic automorphism is ergodic. But, the converse is false. The following matrix gives an example of an ergodic but non hyperbolic automorphism of $\mathbf{T}^{4}$ :

$$
\left(\begin{array}{cccc}
0 & 0 & 0 & -1 \\
1 & 0 & 0 & 2 \\
0 & 1 & 0 & 0 \\
0 & 0 & 1 & 2
\end{array}\right) .
$$

Such an automorphism is said to be quasi-hyperbolic. In the following, we consider an ergodic toral algebraic automorphism $T$ non necessarily hyperbolic. We denote by $\tilde{T}: \mathbf{R}^{n} \rightarrow \mathbf{R}^{n}$ the linear map associated to $T$ and $S$ the linear map transposed to $\tilde{T}$. We denote by $E_{u}$ (resp. $E_{s}$ and $E_{e}$ ) the $S$-stables spaces associated to the eigenvalues of modulus $>1$ (resp. $<1$ et $=1$ ) and $S$. We denote by $m_{u}, m_{s}$ and $m_{e}$ the dimensions of the spaces $E_{u}, E_{s}$ and $E_{e}$ (respectively). For any vector $\alpha$ in $\mathbf{R}^{n}$, we denote by $\left(\alpha_{u}, \alpha_{s}, \alpha_{e}\right)$ the unique element of $E_{u} \times E_{s} \times E_{e}$ such that $\alpha=\alpha_{u}+\alpha_{s}+\alpha_{e}$. Let $v_{1}, \ldots, v_{n}$ be a basis of eigenvectors for $S$. We denote by $\|\cdot\|^{\prime}$ the norm of the supremum in the basis $v_{1}, \ldots, v_{n}$. The following result insures us that the quasi-hyperbolic toral algebraic automorphisms are not $\alpha$-mixing. We recall that the coefficient of $\alpha$-mixing of two $\sigma$-algebras $\mathcal{A}$ and $\mathcal{B}$ contained in the (completed) Borel $\sigma$-algebra is the real number $\alpha(\mathcal{A}, \mathcal{B})$ given by:

$$
\alpha(\mathcal{A}, \mathcal{B}):=\sup _{A \in \mathcal{A}} \sup _{B \in \mathcal{B}}|\nu(A \cap B)-\nu(A) \nu(B)|
$$

Proposition 4.1.1 [3,12]. Let $T$ be a quasihyperbolic algebraic automorphism of the torus $\mathbf{T}^{n}$ and $\mathcal{P}$ be a partition of the torus $\mathbf{T}^{n}$ in a finite number of atoms, the diameters of which are small enough. We denote by $\mathcal{A}_{0}$ the $\sigma$-algebra generated by the partition $\bigvee_{k \geq 0} T^{-k}(\mathcal{P})$ and $\mathcal{B}_{m}$ the $\sigma$-algebra generated by the partition $\bigvee_{k \geq m} T^{k}(\mathcal{P})$. There exists a real number $\beta>0$ such that, for any integer $m \geq 0$, we have $\alpha\left(\mathcal{A}_{0}, \mathcal{B}_{m}\right)>\beta$. Consequently, $\mathcal{P}$ is not $\alpha$-mixing. 
Therefore, Kifer's method does not apply in this case, for the filtration generated by the partition $\mathcal{P}$. Let $\eta \in] 0 ; 1[$ be a real number. We prove the following result:

Theorem 4.1.2. The space $V=\mathcal{H}_{\eta}$ endowed with the norm $\|\cdot\|$ (defined by $\|f\|=\|f\|_{\infty}+C_{f}^{(\eta)}$, where $C_{f}^{(\eta)}$ is the Hölder coefficient of order $\eta$ of the function $f$ ) satisfies the multiple decorrelation property.

The two following results are consequences of this result:

Theorem 4.1.3. Let $T$ be an ergodic (algebraic) automorphism of the torus $\mathbf{T}^{n}$. Then, the conclusion of Theorem 2.1.3 holds for the system $(\Omega, \nu, \sigma)=\left(\mathbf{T}^{n}, \nu, T\right)$ and for $F: \mathbf{R}^{d} \times \mathbf{T}^{n} \rightarrow \mathbf{R}^{d}$ if $F$ is $C_{b}^{2,0}$ and if $F$ is uniformly Hölder of order $\eta$ in the second variable.

Theorem 4.1.4. Let $T$ be an ergodic automorphism of the torus $\mathbf{T}^{n}$. We consider the suspension flow ( $\mathcal{M}, \mu$, $\left.\left(Y_{t}\right)_{t}\right)$ defined over $\left(\mathbf{T}^{n}, \nu, T\right)$ by a function $\left.\tau: \mathbf{T}^{n} \rightarrow\right] 0 ;+\infty[$ Hölder of order $\eta$. Then, the conclusion of Theorem 3.4.4 holds for the flow $\left(Y_{t}\right)_{t}$ and for the function $f: \mathbf{R}^{d} \times \mathcal{M} \rightarrow \mathbf{R}^{d}$ if $f$ is $\mathcal{C}_{b}^{(2)}$ and such that the function $F: \mathbf{R}^{d} \times \Omega \rightarrow \mathbf{R}^{d}$ given by $F(x, \omega):=\int_{0}^{\tau(\omega)} f(x,(\omega, s)) \mathrm{d} s$ is uniformly Hölder of order $\eta$ in the second variable.

Proof of the Theorem 4.1.2. We fix two integers $m \geq 1$ and $m^{\prime} \geq 1$ and a real number $r>1$. Let an integer $N \geq 1$ and $\left(m+m^{\prime}\right)$ elements of $V$ denoted $f^{(1)}, \ldots, f^{(m)}, g^{(1)}, \ldots, g^{\left(m^{\prime}\right)}$ and $\left(m+m^{\prime}\right)$ integers $k_{1}, k_{2}, \ldots, k_{m}, l_{1}, l_{2}, \ldots, l_{m^{\prime}}$ with $0 \leq k_{1} \leq k_{2} \leq \cdots \leq k_{m}$ and $0 \leq l_{1} \leq l_{2} \leq \cdots \leq l_{m^{\prime}}$. We define the functions $F:=\prod_{i=1}^{m} f^{(i)} \circ T^{k_{i}}$ and $G:=\prod_{j=1}^{m^{\prime}} g^{(j)} \circ T^{l_{j}}$. We consider the quantity $\operatorname{Cov}\left(F ; G \circ T^{N}\right)$. We shall approach each function $f^{(i)}$ and $g^{(j)}$ by a trigonometrical polynom, using the Fejer kernel. The proof of the theorem shall be given in several steps. First, we give the following general result, a proof of which is given in [13] and [9]. Its corollary shall be useful in the following:

Lemma 4.1.5. Let $S_{0}: \mathbf{R}^{n} \rightarrow \mathbf{R}^{n}$ be a linear map given by a matrix with integer coefficients and let $\|\cdot\|_{0}$ be a norm on $\mathbf{R}^{n}$. Let $V$ and $W$ be two vector sub-spaces of $\mathbf{R}^{n} S_{0}$-stables such that $\mathbf{R}^{n}=V \oplus W$ and such that the eigenvalues of $\left(S_{0}\right)_{\mid V}$ are distinct from those of $\left(S_{0}\right)_{\mid W}$. If $V \cap \mathbf{Z}^{n}=\{0\}$, then there exists a real number $K>0$ such that, for any non zero integer vector $k$ in $\mathbf{Z}^{n}$, we have

$$
\inf _{v \in V}\|k-v\|_{0} \geq K\|k\|_{0}^{-\operatorname{dim}(V)}
$$

Corollary. Since $T$ is ergodic, we have $\mathbf{Z}^{n} \cap\left(E_{e} \oplus E_{s}\right)=\emptyset$. Consequently, there exist two constants $K_{(e, s)}>0$ and $K_{(e, s)}^{\prime}>0$ such that, for all nonnul integer vector $\alpha \in \mathbf{Z}^{n}$, we have

$$
\left\|\alpha_{u}\right\|^{\prime} \geq \frac{K_{(e, s)}}{\left(\|\alpha\|^{\prime}\right)^{m_{e}+m_{s}}} \geq \frac{K_{(e, s)}^{\prime}}{\left(\|\alpha\|^{\prime}\right)^{n}} .
$$

Lemma 4.1.6. There exists a constant $\tilde{C}_{0}>0$ such that, for any integer $M \geq 1$ and any function $f: \mathbf{T}^{n} \rightarrow \mathbf{R}$ Hölder continuous of order $\eta$ with Hölder coefficient $C_{f}^{(\eta)}$, there exists a trigonometrical polynom $f_{M}$ of degree bounded by $M$ (for the norm of the supremum in the canonical basis) such that we have

$$
\left\|f_{M}\right\|_{\infty} \leq\|f\|_{\infty} \text { and }\left\|f-f_{M}\right\|_{\infty} \leq \tilde{C}_{0} C_{f}^{(\eta)} M^{-\frac{\eta}{2+\eta}}
$$


Proof. We use the Fejer kernel $k_{N}(x):=\frac{1}{N}\left(\frac{\sin (\pi N x)}{\sin (\pi x)}\right)^{2}$. We put $K_{N}\left(x_{1}, \ldots, x_{n}\right):=\prod_{i=1}^{n} k_{N}\left(x_{i}\right)$. Then we have, for any integer $N \geq 1$ and any real number $\delta>0$,

$$
\begin{aligned}
\left|f(x)-K_{N} * f(x)\right| & =\left|f(x)-\int_{\mathbf{T}^{n}} f(x-t) K_{N}\left(t_{1}, \ldots, t_{n}\right) \mathrm{d} t_{1} \ldots \mathrm{d} t_{n}\right| \\
& \leq \int_{\mathbf{T}^{n}}|f(x)-f(x-t)| K_{N}\left(t_{1}, \ldots, t_{n}\right) \mathrm{d} t_{1} \ldots \mathrm{d} t_{n} \\
& \leq \int_{\mathbf{T}^{n}}|f(x)-f(x-t)| k_{N}\left(t_{1}\right) \cdot \ldots \cdot k_{N}\left(t_{n}\right) \mathrm{d} t_{1} \ldots \mathrm{d} t_{n} \\
& \leq \int_{\mathbf{T}^{n}} C_{f}^{(\eta)}|t|^{\eta} k_{N}\left(t_{1}\right) \cdot \ldots \cdot k_{N}\left(t_{n}\right) \mathrm{d} t_{1} \ldots \mathrm{d} t_{n} \\
& \leq n ! \int_{0 \leq\left|t_{1}\right| \leq \ldots \leq\left|t_{n}\right| \leq \frac{1}{2}} C_{f}^{(\eta)}\left|t_{n}\right|^{\eta} k_{N}\left(t_{1}\right) \cdot \ldots \cdot k_{N}\left(t_{n}\right) \mathrm{d} t_{1} \ldots \mathrm{d} t_{n} \\
& \leq n ! C_{f}^{(\eta)} \int_{\mathbf{T}}\left|t_{n}\right|^{\eta} k_{N}\left(t_{n}\right) \mathrm{d} t_{n} \\
& \leq n ! C_{f}^{(\eta)}\left(\int_{|t| \leq \delta}|t|^{\eta} k_{N}(t) \mathrm{d} t+\int_{\delta<|t| \leq \frac{1}{2}}|t|^{\eta} k_{N}(t) \mathrm{d} t\right) \\
& \leq n ! C_{f}^{(\eta)}\left(\delta^{\eta}+\int_{\delta<|t| \leq \frac{1}{2}}|t|^{\eta} k_{N}(t) \mathrm{d} t\right) .
\end{aligned}
$$

For any real number $t$ such that $\delta<|t| \leq \frac{1}{2}$, we have $k_{N}(t) \leq \frac{1}{N(\sin (\pi t))^{2}} \leq \frac{1}{N(\sin (\pi \delta))^{2}} \leq \frac{1}{N 4 \delta^{2}}$. We get $\left|f(x)-K_{N} * f(x)\right| \leq n ! C_{f}^{(\eta)}\left(\delta^{\eta}+\frac{1}{2^{\eta}} \frac{1}{N 4 \delta^{2}}\right)$. We conclude by taking $\delta=\delta_{N}=N^{-\frac{1}{2+\eta}}$.

Continuation of the proof of Theorem 4.1.2. For any integer $M \geq 1$ and any $i=1, \ldots, m$ (resp. any $j=1, \ldots, m^{\prime}$ ), we denote by $f_{M}^{(i)}$ (resp. $g_{M}^{(j)}$ ) the trigonometrical polynoms defined as in the previous lemma for $f=f^{(i)}$ (resp. $\left.f=g^{(j)}\right)$. For any integer $M \geq 1$, we denote by $F_{M}:=\prod_{i=1}^{m} f_{M}^{(i)} \circ T^{k_{i}}$ and $G_{M}:=\prod_{j=1}^{m^{\prime}} g_{M}^{(j)} \circ T^{l_{j}}$. Then we have

$$
\left\|F-F_{M}\right\|_{\infty} \leq \tilde{C}_{0}\left(\prod_{i=1}^{m}\left\|f^{(i)}\right\|\right) M^{-\frac{\eta}{2+\eta}} \text { and }\left\|G-G_{M}\right\|_{\infty} \leq \tilde{C}_{0}\left(\prod_{j=1}^{m^{\prime}}\left\|g^{(j)}\right\|\right) M^{-\frac{\eta}{2+\eta}}
$$

Now, we establish the following lemma:

Lemma 4.1.7. Let two integers $m \geq 1$ and $m^{\prime} \geq 1$, two real numbers $r>1$ and $\left.\gamma \in\right] 0 ; \frac{1}{2 n}[$ be given. There exist an integer $N_{0} \geq 1$ and a real number $\left.r_{0} \in\right] 0 ; 1$ [ such that for any integers $0 \leq k_{1} \leq \cdots \leq k_{m}, 0 \leq l_{1} \leq \cdots \leq l_{m^{\prime}}$ and any integer $N$ satisfying $N \geq r \max \left(k_{m}, l_{m^{\prime}}\right)$ and $N-\max \left(k_{m}, l_{m^{\prime}}\right) \geq N_{0}$, for any trigonometrical polynoms $P^{(1)}, \ldots, P^{(m)}$ and $Q^{(1)}, \ldots, Q^{\left(m^{\prime}\right)}$ of degrees (for the norm $\|\cdot\|^{\prime}$ ) bounded by $r_{0}^{-\gamma\left(N-k_{m}\right)}$, we have

$$
\operatorname{Cov}\left(\prod_{i=1}^{m} P^{(i)} \circ T^{k_{i}}, \prod_{j=1}^{m^{\prime}} Q^{(j)} \circ T^{l_{j}+N}\right)=0
$$

Proof. We denote by $r_{u}$ the spectral radius of $S^{-1} \mid E_{u}$. We fix a real number $\left.r_{0} \in\right] r_{u} ; 1[$. Then, there exists an integer $N_{1} \geq 1$ such that, for any integer $N \geq N_{1}$, we have $\left\|S^{-N} \mid E_{u}\right\|^{\prime} \leq r_{0}^{N}$. Put $k_{i}^{\prime}:=\left\lfloor\frac{N-k_{m}}{2}\right\rfloor+k_{m}-k_{m-i+1}$, 
$\tilde{P}^{(i)}:=P^{(m-i+1)}, l_{j}^{\prime}:=l_{j}+\left\lceil\frac{N-k_{m}}{2}\right\rceil$ et $\tilde{Q}^{(j)}:=Q^{(j)}$. Therefore, we have

$$
\begin{aligned}
\operatorname{Cov}\left(\prod_{i=1}^{m} P^{(i)} \circ T^{k_{i}}, \prod_{j=1}^{m^{\prime}} Q^{(j)} \circ T^{l_{j}+N}\right) & =\operatorname{Cov}\left(\prod_{i=1}^{m} P^{(i)} \circ T^{k_{i}-k_{m}-\left\lfloor\frac{N-k_{m}}{2}\right\rfloor}, \prod_{j=1}^{m^{\prime}} Q^{(j)} \circ T^{l_{j}+N-k_{m}-\left\lfloor\frac{N-k_{m}}{2}\right\rfloor}\right) \\
& =\operatorname{Cov}\left(\prod_{i=1}^{m} \tilde{P}^{(i)} \circ T^{-k_{i}^{\prime}}, \prod_{j=1}^{m^{\prime}} \tilde{Q}^{(j)} \circ T^{l_{j}^{\prime}}\right)
\end{aligned}
$$

and $\left\lfloor\frac{N-k_{m}}{2}\right\rfloor=k_{1}^{\prime} \leq k_{2}^{\prime} \leq \cdots \leq k_{m}^{\prime}$ and $\left\lceil\frac{N-k_{m}}{2}\right\rceil \leq l_{1}^{\prime} \leq l_{2}^{\prime} \leq \cdots \leq l_{m^{\prime}}^{\prime}=\left\lceil\frac{N-k_{m}}{2}\right\rceil+l_{m^{\prime}}$. The frequencies appearing in the trigonometrical polynom $\prod_{i=1}^{m^{2}} \tilde{P}^{(i)} \circ T^{-k_{i}^{\prime}}$ are of the form $X_{\xi}^{-}:=\sum_{i=1}^{m} S^{-k_{i}^{\prime}} \xi_{i}$, where each $\xi_{i}$ is an integer vector of $\mathbf{Z}^{n}$ such that $\left\|\xi_{i}\right\|^{\prime} \leq r_{0}{ }^{-\gamma\left(N-k_{m}\right)}$. In the same way, the frequencies appearing in the trigonometrical polynom $\prod_{j=1}^{m^{\prime}} \tilde{Q}^{(j)} \circ T^{l_{j}^{\prime}}$ are of the form $X_{\eta}^{+}:=\sum_{j=1}^{m^{\prime}} S^{l_{j}^{\prime}} \eta_{j}$ where each $\eta_{j}$ is an integer vector of $\mathbf{Z}^{n}$ such that $\left\|\eta_{j}\right\|^{\prime} \leq r_{0}{ }^{-\gamma\left(N-k_{m}\right)}$. Therefore, to show that we have:

$$
\operatorname{Cov}\left(\prod_{i=1}^{m} \tilde{P}^{(i)} \circ T^{-k_{i}^{\prime}}, \prod_{j=1}^{m^{\prime}} \tilde{Q}^{(j)} \circ T^{l_{j}^{\prime}}\right)=0,
$$

it suffices to show that if $X_{\xi}^{-}+X_{\eta}^{+}=0$, then $X_{\xi}^{-}=X_{\eta}^{+}=0$. Let us consider integer vectors $\xi_{1}, \ldots, \xi_{m}, \eta_{1}, \ldots, \eta_{m^{\prime}} \in$ $\mathbf{Z}^{n}$, the coordinates of which in the basis $\left(v_{1}^{\prime}, \ldots, v_{n}^{\prime}\right)$ are less than $r_{0}{ }^{-\gamma\left(N-k_{m}\right)}$ and such that $X_{\xi}^{-}$and $X_{\eta}^{+}$are non zero. Let us suppose $N \geq r \max \left(k_{m}, l_{m^{\prime}}\right)$. We shall see that if $N-\max \left(k_{m}, l_{m^{\prime}}\right)$ is large enough, then we have

If $N-\max \left(k_{m}, l_{m^{\prime}}\right) \geq 2 N_{1}$, then we have

$$
\left\|\left(X_{\eta}^{+}\right)_{u}\right\|^{\prime}>\left\|\left(X_{\xi}^{-}\right)_{u}\right\|^{\prime}
$$

$$
\begin{aligned}
\left\|\left(X_{\xi}^{-}\right)_{u}\right\|^{\prime}=\left\|\sum_{i=1}^{m} S^{-k_{i}^{\prime}}\left(\xi_{i}\right)_{u}\right\|^{\prime} \leq \sum_{i=1}^{m}\left\|S^{-k_{i}^{\prime}}\left(\xi_{i}\right)_{u}\right\|^{\prime} & \leq \sum_{i=1}^{m} r_{0}^{k_{i}^{\prime}}\left\|\left(\xi_{i}\right)_{u}\right\|^{\prime} \\
& \leq m r_{0}^{\left\lfloor\frac{N-k_{m}}{2}\right\rfloor}\left\|\left(\xi_{i}\right)_{u}\right\|^{\prime} \leq m r_{0}{ }^{\left(\frac{1}{2}-\gamma\right)\left(N-k_{m}\right)} r_{0}{ }^{-1}
\end{aligned}
$$

On the other hand, there exists a constant $C>0$ such that, for any integer $l \geq 0$, we have $\left\|S_{\mid E_{s} \oplus E_{e}}\right\| \leq C(l+1)^{n}$. We get

$$
\begin{aligned}
\left\|\left(S^{-\left\lceil\frac{N-k_{m}}{2}\right\rceil}\left(X_{\eta}^{+}\right)\right)_{s, e}\right\|^{\prime} & =\left\|\sum_{j=1}^{m^{\prime}} S^{l_{j}^{\prime}-\left\lceil\frac{N-k_{m}}{2}\right\rceil}\left(\eta_{j}\right)_{s, e}\right\|^{\prime} \leq \sum_{j=1}^{m^{\prime}} C\left(l_{j}^{\prime}-\left\lceil\frac{N-k_{m}}{2}\right\rceil+1\right)^{n}\left\|\left(\eta_{j}\right)_{s, e}\right\|^{\prime} \\
& \leq m^{\prime} C\left(l_{m^{\prime}}+1\right)^{n} r_{0}^{-\gamma\left(N-k_{m}\right)} \leq m^{\prime} C^{\prime}\left(\frac{N-k_{m}}{r-1}\right)^{n} r_{0}^{-\gamma\left(N-k_{m}\right)},
\end{aligned}
$$

with $C^{\prime}=C\left(1+\frac{r-1}{2 N_{1}}\right)^{n}$, since we have $N-k_{m} \geq N-\max \left(k_{m}, l_{m^{\prime}}\right) \geq(r-1) \max \left(k_{m}, l_{m^{\prime}}\right) \geq(r-1) l_{m^{\prime}}$ and $N-k_{m} \geq 2 N_{1}>0$. Therefore, there exists a constant $C_{1}>0$ (independent of $N, k_{1}, \ldots, k_{m}, l_{1}, \ldots, l_{m^{\prime}}$ ) such that, under the previous hypotheses, we have

$$
\left\|\left(S^{-\left\lceil\frac{N-k_{m}}{2}\right\rceil}\left(X_{\eta}^{+}\right)\right)_{u}\right\|^{\prime} \geq \frac{C_{1}}{\left(\frac{N-k_{m}}{r-1}\right)^{n^{2}} r_{0}^{-n \gamma\left(N-k_{m}\right)}} .
$$


Indeed, let $\tilde{C}>0$ be a real number. If we have $\left\|\left(S^{-\left\lceil\frac{N-k_{m}}{2}\right\rceil}\left(X_{\eta}^{+}\right)\right)_{u}\right\|^{\prime} \leq \frac{\tilde{C}}{\left(\frac{N-k_{m}}{r-1}\right)^{n^{2}} r_{0}^{-n \gamma\left(N-k_{m}\right)}}$, then there exists a constant $C_{2}>0$ such that we have $\left\|S^{-\left\lceil\frac{N-k_{m}}{2}\right\rceil}\left(X_{\eta}^{+}\right)\right\|^{\prime} \leq C_{2}\left(\frac{N-k_{m}}{r-1}\right)^{n} r_{0}^{-\gamma\left(N-k_{m}\right)}$. According to the corollary of Lemma 4.1.5, we get $\left\|\left(S^{-\left\lceil\frac{N-k_{m}}{2}\right\rceil}\left(X_{\eta}^{+}\right)\right)_{u}\right\|^{\prime} \geq \frac{K_{(e, s)}^{\prime}}{C_{2}^{n}\left(\frac{N-k_{m}}{r-1}\right)^{n^{2}} r_{0}^{-n \gamma\left(N-k_{m}\right)}}$. The constant $C_{1}:=\min \left(\tilde{C}, \frac{K_{(e, s)}^{\prime}}{C_{2}{ }^{n}}\right)$ is thus suitable. So we have

$$
\left\|\left(X_{\eta}^{+}\right)_{u}\right\|^{\prime} \geq \frac{C_{1} r_{0}-\left\lceil\frac{N-k_{m}}{2}\right\rceil}{\left(\frac{N-k_{m}}{r-1}\right)^{n^{2}} r_{0}^{-n \gamma\left(N-k_{m}\right)}} \geq \frac{C_{1} r_{0}\left(\gamma n-\frac{1}{2}\right)\left(N-k_{m}\right)}{\left(\frac{N-k_{m}}{r-1}\right)^{n^{2}}} .
$$

Since we have $\gamma n<\frac{1}{2}$, if $N-k_{m}$ is large enough, we have

$$
\frac{C_{1} r_{0}\left(\gamma n-\frac{1}{2}\right)\left(N-k_{m}\right)}{\left(\frac{N-k_{m}}{r-1}\right)^{n^{2}}}>m r_{0}{ }^{\left(\frac{1}{2}-\gamma\right)\left(N-k_{m}\right)} r_{0}{ }^{-1}
$$

End of the proof of Theorem 4.1.2. The equivalence of the norms in finite dimension insures us the existence of a real number $c>0$ such that $\|\cdot\|^{\prime} \leq c|\cdot|_{\infty}$. Put $N_{0}^{\prime}:=\max \left(N_{0}, \frac{\ln (c)}{\gamma\left(-\ln \left(r_{0}\right)\right)}\right)$.

- If $N \leq r \max \left(k_{m}, l_{m^{\prime}}\right)+N_{0}^{\prime}$, then we have

$$
|\operatorname{Cov}(F, G)| \leq \prod_{i=1}^{m}\left\|f^{(i)}\right\|_{\infty} \prod_{j=1}^{m^{\prime}}\left\|g^{(j)}\right\|_{\infty} \leq \prod_{i=1}^{m}\left\|f^{(i)}\right\| \prod_{j=1}^{m^{\prime}}\left\|g^{(j)}\right\| r_{0} \frac{\gamma \eta\left(N-r \max \left(k_{m}, l_{m^{\prime}}\right)\right)}{2+\eta} r_{0}{ }^{-\frac{\gamma \eta N_{0}^{\prime}}{2+\eta}}
$$

- Now, let us suppose $N \geq r \max \left(k_{m}, l_{m^{\prime}}\right)+N_{0}^{\prime}$. Put $M:=\left\lfloor\frac{r_{0}-\gamma\left(N-k_{m}\right)}{c}\right\rfloor$. We have

$$
\operatorname{Cov}(F, G)=\operatorname{Cov}\left(F-F_{M}, G\right)+\operatorname{Cov}\left(F_{M}, G-G_{M}\right)+\operatorname{Cov}\left(F_{M}, G_{M}\right) .
$$

Since $M \geq 1$, we have $M \geq \frac{r_{0}-\gamma\left(N-k_{m}\right)}{2 c}$ and, therefore, $\left\|F-F_{m}\right\|_{\infty}$ and $\left\|G-G_{M}\right\|_{\infty}$ are less than:

$$
\tilde{C}_{0}(2 c)^{\frac{\eta}{2+\eta}}\left(\prod_{i=1}^{m}\left\|f^{(i)}\right\|\right) r_{0} \frac{\gamma \eta\left(N-k_{m}\right)}{2+\eta} .
$$

Thus, $\left|\operatorname{Cov}\left(F-F_{M}, G\right)\right|$ and $\left|\operatorname{Cov}\left(F_{M}, G-G_{M}\right)\right|$ are less than:

$$
\tilde{C}_{0}(2 c)^{\frac{\eta}{2+\eta}}\left(\prod_{i=1}^{m}\left\|f^{(i)}\right\|\right) r_{0} \frac{\gamma \eta\left(N-k_{m}\right)}{2+\eta}\left(\prod_{j=1}^{m^{\prime}}\left\|g^{(j)}\right\|\right) .
$$

On the other hand, since the degrees of $F_{M}$ and $G_{M}$ for the norm $\|\cdot\|^{\prime}$ are less than $c M$, we have $\operatorname{Cov}\left(F_{M}, G_{M}\right)=0$, according to Lemma 4.1.7.

\subsection{Sinai dispersive billiard with finite horizon}

In this section we summarize results obtained in [14]. Some details are given in Appendix B. Stochastical properties (ergodicity, $K$-system, CLT, exponential rate of decorrelation) of the system we consider here have been studied in $[5-7,14,17,18]$ and in many other articles. We consider a compact subset $Q$ of $\mathbf{T}^{2}$ (with 
connected interior), the complement of which is the finite union of strictly convex open sets (the closure of which are pairwise disjoints). We suppose that the boundary $\partial Q$ of $Q$ is $C^{3}$ with curvature $\kappa$ never null. Two examples of such domain $Q$ are drawn (in white) in the following picture. For any $q \in \partial Q$, we denote by $\vec{n}(q)$ the unitary normal vector to $\partial Q$ at $q$, oriented to the inside of $Q$. We are interested in the behavior of a pinpoint particle moving in $Q$ with unitary speed and elastic reflections off $\partial Q$.

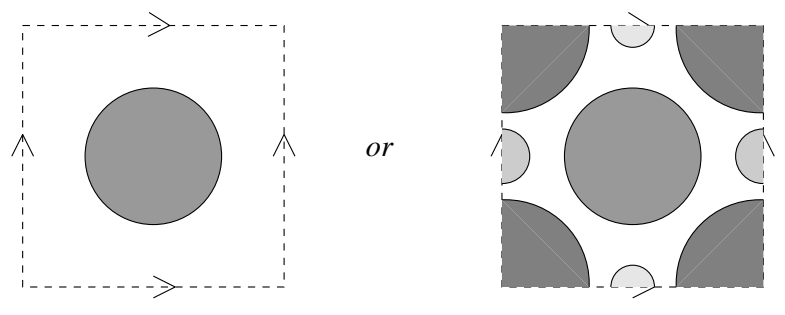

FIGURE 1

Here, the set of configurations is the set $Q_{1}$ given by

$$
Q_{1}:=\mathcal{T}^{1} \stackrel{\circ}{Q} \cup M, \text { with } M:=\left\{x=(q, \vec{v}) \in \mathcal{T}^{1} Q: q \in \partial Q,\langle\vec{n}(q), \vec{v}\rangle \geq 0\right\}
$$

where $\mathcal{T}^{1} A$ denote the unitary tangent bundle to $A$. The billiard flow in $Q$ is the flow $\left(S_{t}\right)_{t}$ on $Q_{1}$ given by $S_{t}(q, \vec{v})=\left(q_{t}, \vec{v}_{t}\right)$, where $\left(q_{t}, \vec{v}_{t}\right)$ is the couple position-speed at time $t$ of a particle that was at the position $q$ with the speed $\vec{v}$ at time 0 . This flow preserves the normalised Lebesgue measure $\mu_{1}$ on $Q_{1}$. It is a classical result that this flow can be represented by the suspension flow over $(M, \nu, T)$ defined by the function $\left.\tau^{+}: M \rightarrow\right] 0 ;+\infty[$ where:

- $M$ is the set defined previously, corresponding to the set of configurations at the time just after a reflection;

- $\nu$ is the Borel probability measure on $M$ proportional to the measure given by $\cos (\varphi) \mathrm{d} r \mathrm{~d} \varphi$ where $\varphi$ is the angular measure in $\left[-\frac{\pi}{2} ; \frac{\pi}{2}\right]$ of the angle between $\vec{n}(q)$ and $\vec{v}$ and where $r$ is the curvilinear absciss of $q$ on the connected component of $\partial Q$ to which it belongs;

- $T$ is the transformation that maps a configuration $(q, \vec{v}) \in M$ of a particle, at the time after a reflection, to the configuration $\left(q^{\prime}, \vec{v}^{\prime}\right)$ of this particle at the time after the next reflection;

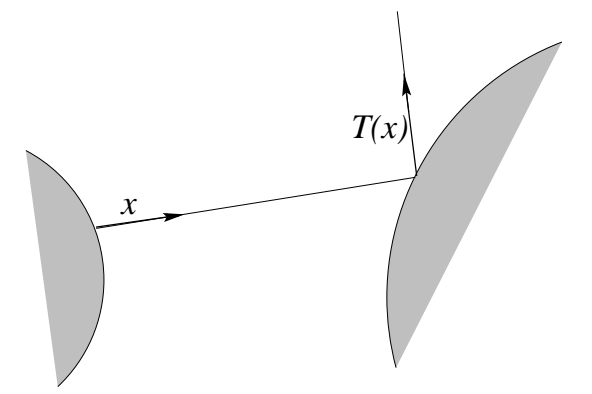

- $\tau^{+}(q, \vec{v})$ is the distance covered by a particle at position $q \in \partial Q$ with speed $\vec{v}$ until the next reflection off $\partial Q$.

It is well-known that the probability measure $\nu$ is $T$-invariant. The dynamical system $(M, \nu, T)$ is called the billiard system in $Q$. We shall suppose here that the billiard in $Q$ has finite horizon, i.e. that the function $\tau^{+}$is uniformly bounded. In the first picture (Fig. 1), only the second example has finite horizon. We denote by $R_{0}$ the set of vectors tangent to $\partial Q: R_{0}:=\{(q, \vec{v}) \in M:\langle\vec{n}(q), \vec{v}\rangle=0\}$. For any integer $k$, we define $R_{k}:=T^{k}\left(R_{0}\right)$. For any $-\infty \leq k \leq l \leq+\infty$, we denote $R_{k, l}:=\bigcup_{j=k}^{l} R_{j}$. The study of the billiard system $(M, \nu, T)$ is complicated by the existence of singularities for $T$ corresponding to points in $R_{-1}$ (cf. picture). 


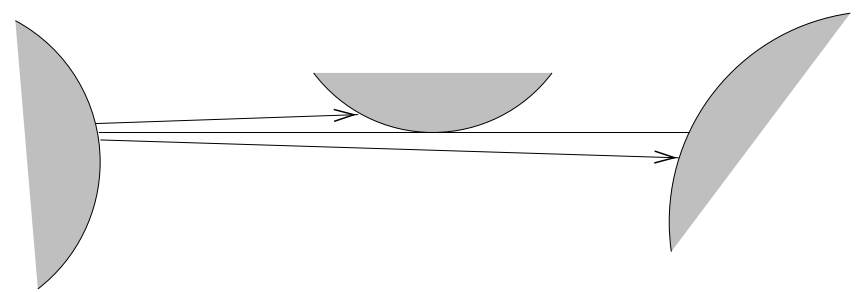

But, it is well known that, for any integer $k \geq 1, T^{k}$ defines a $C^{1}$ diffeomorphism from $M \backslash R_{-k, 0}$ onto $M \backslash R_{0, k}$.

For any real number $\eta>0$ and any integer $m \geq 0$, we denote by $\mathcal{H}_{\eta, m}$ the set of the bounded functions $\phi: M \rightarrow \mathbf{R}$ for which the following quantity is finite:

$$
C_{\phi}=C_{\phi}^{(\eta, m)}:=\sup _{C \in \mathcal{C}_{m}} \sup _{x, y \in C, x \neq y} \frac{|\phi(x)-\phi(y)|}{\left(\max \left(d(x, y), \ldots, d\left(T^{m}(x), T^{m}(y)\right)\right)^{\eta}\right.},
$$

where $\mathcal{C}_{m}$ is the set of the connected components of $M \backslash R_{-m, 0}$ and where $d$ is the metric defined on each connected component of $M$ by $d\left((q, \vec{v}),\left(q^{\prime}, \vec{v}^{\prime}\right)\right)=\sqrt{\left|r-r^{\prime}\right|^{2}+\left|\varphi-\varphi^{\prime}\right|}$ if $r$ (resp. $\left.r^{\prime}\right)$ is the curvilinear absciss of $q$ (resp. $\left.q^{\prime}\right)$ and if $\varphi$ (resp. $\left.\varphi^{\prime}\right)$ is the angular measure in $\left[-\frac{\pi}{2} ; \frac{\pi}{2}\right]$ between $\vec{n}(q)$ and $\vec{v}$ (resp. $\vec{v}^{\prime}$ ). This space can be understood as the space of functions $\eta$-Hölder continuous in the $m$ future coordinates.

Examples. If $\phi: M \rightarrow \mathbf{R}$ is Hölder continuous of order $\eta$ on each connected component of $M$, then $\phi \circ T^{m}$ is maybe not Hölder continuous but is in $\mathcal{H}_{\eta, m}$. The function $\tau^{+}$is in $\mathcal{H}_{1,1}$.

We suppose now that the flow $\left(\mathcal{M}, \mu,\left(Y_{t}\right)_{t}\right)$ is the billiard flow $\left(Q_{1}, \mu_{1},\left(S_{t}\right)_{t}\right)$ in the domain $Q$ and that the dynamical system $(\Omega, \nu, \sigma)$ is the billiard system $(M, \nu, T)$ in $Q$. We observe that, contrarily to the previous examples, here the functional spaces we are interested in are not stable by the transformation $T$. Nevertheless, if $g$ is in $\mathcal{H}_{\eta, m}$, then $g \circ T$ is in $\mathcal{H}_{\eta, m+1}$ and the controls we obtain are, in some way, uniform in $m$. Indeed, using the method developed by Young in [18], we have the following result a proof of which is given in [15]. To be complete, we give this proof in Appendix B.

Proposition 4.2.1 (Strong decorrelation property [15]). Let $\eta>0$ and $\kappa \in] 0 ; \frac{1}{2}$ [ be two real numbers. There exist constants $C_{\eta, \kappa}>0$ and $\left.\alpha_{\eta, \kappa} \in\right] 0 ; 1\left[\right.$ such that, for any integers $m_{1}, m_{2} \geq 0$, for any functions $\phi$ and $\psi$ in $\mathcal{H}_{\eta, m_{1}}$ and in $\mathcal{H}_{\eta, m_{2}}$ respectively and for any integer $n \geq 0$, we have

$$
\left|\operatorname{Cov}\left(\phi, \psi \circ T^{n}\right)\right| \leq C_{\eta, \kappa}\left(\|\phi\|_{\infty}+C_{\phi}^{\left(\eta, m_{1}\right)}\right)\left(\|\psi\|_{\infty}+C_{\psi}^{\left(\eta, m_{2}\right)}\right) \alpha_{\eta, \kappa}{ }^{n-\frac{m_{1}}{1-2 \kappa}}
$$

An other application of this result is the CLT with a rate of convergence in $O\left(n^{-\frac{1}{4}+\varepsilon}\right)$ for functions in $\mathcal{H}_{\eta, m}$ (cf. [15]). Moreover, we mention the fact that such a result can be established for any dynamical system to which Young's method can be applied. Indeed, calculations done in Section I.4 of [18] can be modified as in Appendix B of this redaction. The following result insures us that, for any real number $\eta>0$ and any integer $m_{0} \geq 0$, the normed vector space $\left(\mathcal{H}_{\eta, m_{0}},\|\cdot\|_{\eta, m_{0}}\right)$ satisfies the multiple decorrelation property with $\|f\|_{\eta, m_{0}}:=\|f\|_{\infty}+C_{f}^{\left(\eta, m_{0}\right)}$.

Corollary. For any $\kappa \in] 0 ; \frac{1}{2}\left[\right.$, any real number $\eta>0$, any integer $m_{0} \geq 0$, for any integer $N \geq 0$ and any increasing sequences of nonnegative integers $\left(k_{1}, \ldots, k_{m}\right)$ and $\left(l_{1}, \ldots, l_{m^{\prime}}\right)$, any $g_{1}, \ldots, g_{m}, h_{1}, \ldots, h_{m^{\prime}}$ in $\mathcal{H}_{\eta, m_{0}}$, we have

$$
\left|\operatorname{Cov}\left(\prod_{i=1}^{m} g_{i} \circ T^{k_{i}} ; \prod_{j=1}^{m^{\prime}} h_{j} \circ T^{N+l_{j}}\right)\right| \leq C_{\eta, \kappa} \alpha_{\eta, \kappa}-\frac{m_{0}}{1-2 \kappa} \prod_{i=1}^{n}\left\|g_{i}\right\|_{\eta, m_{0}} \cdot \prod_{j=1}^{m}\left\|h_{j}\right\|_{\eta, m_{0}} \cdot \alpha_{\eta, \kappa}{ }^{N-\frac{k_{m}}{1-2 \kappa}} .
$$


Proof. Function $G:=\prod_{i=1}^{m} g_{i} \circ T^{k_{i}}$ is in $\mathcal{H}_{\eta, m_{0}+k_{m}}$ and we have:

$$
\left\|\prod_{i=1}^{m} g_{i} \circ T^{k_{i}}\right\|_{\eta, m_{0}+k_{m}} \leq \prod_{i=1}^{m}\left\|g_{i}\right\|_{\eta, m_{0}} .
$$

On the other hand, function $H:=\prod_{j=1}^{m^{\prime}} h_{j} \circ T^{l_{j}}$ is in $\mathcal{H}_{\eta, m_{0}+l_{m^{\prime}}}$ and we have $\left\|\prod_{j=1}^{m^{\prime}} h_{j} \circ T^{l_{j}}\right\|_{\eta, m_{0}+l_{m^{\prime}}} \leq$ $\prod_{j=1}^{m^{\prime}}\left\|h_{j}\right\|_{\eta, m_{0}}$. Indeed, we have $\|G\|_{\infty} \leq \prod_{i=1}^{m}\left\|g_{i}\right\|_{\infty}$ and $\|H\|_{\infty} \leq \prod_{j=1}^{m^{\prime}}\left\|h_{j}\right\|_{\infty}$. Moreover, for any $i=1, \ldots, m$ and any $j=1, \ldots, m^{\prime}$, we have $C_{g_{i} \circ T^{k_{i}}}^{\left(\eta, m_{m}+k_{m}\right)} \leq C_{g_{i}}^{\left(\eta, m_{0}\right)}$ and $C_{h_{j} \circ T^{l_{j}}}^{\left(\eta, m_{0}+l_{m^{\prime}}\right)} \leq C_{h_{j}}^{\left(\eta, m_{0}\right)}$. Therefore we have

$$
C_{G}^{\left(\eta, m_{0}+k_{m}\right)} \leq \sum_{i=1}^{m}\left(C_{g_{i}}^{\left(\eta, m_{0}\right)} \prod_{i^{\prime} \neq i}\left\|g_{i^{\prime}}\right\|_{\infty}\right) \quad \text { and } C_{H}^{\left(\eta, m_{0}+l_{m^{\prime}}\right)} \leq \sum_{j=1}^{m^{\prime}}\left(C_{h_{j}}^{\left(\eta, m_{0}\right)} \prod_{j^{\prime} \neq j}\left\|h_{j^{\prime}}\right\|_{\infty}\right)
$$

So, according to the previous proposition, we have

$$
\begin{aligned}
\left|\operatorname{Cov}\left(G ; H \circ T^{N}\right)\right| & \leq C_{\eta, \kappa}\|G\|_{\eta, m_{0}+k_{m}}\|H\|_{\eta, m_{0}+l_{m^{\prime}}} \alpha_{\eta, \kappa}{ }^{N-\frac{m_{0}+k_{m}}{1-2 \kappa}} \\
& \leq C_{\eta, \kappa} \prod_{i=1}^{m}\left\|g_{i}\right\|_{\eta, m_{0}} \prod_{j=1}^{m^{\prime}}\left\|h_{j}\right\|_{\eta, m_{0}} \alpha_{\eta, \kappa}{ }^{N-\frac{m_{0}+k_{m}}{1-2 \kappa}} .
\end{aligned}
$$

Theorem 4.2.2. Let $\eta>0$ be a real number and $m_{0} \geq 0$ be an integer. The conclusion of Theorem 2.1.3 holds for the billiard system $(\Omega, \nu, \sigma)=(M, \nu, T)$ in the domain $Q$ of the torus $\mathbf{T}^{2}$ and for a function $F: \mathbf{R}^{d} \times M \rightarrow \mathbf{R}^{d}$ $C_{b}^{2, *}$, such that the coordinates of $F$ are uniformly in $\mathcal{H}_{\eta, m_{0}}$ in the second variable, i.e.

$$
\sup _{x \in \mathbf{R}^{d}} \sup _{i=1, \ldots, d} C_{F_{i}(x, \cdot)}^{\left(\eta, m_{0}\right)}<+\infty
$$

Theorem 4.2.3. Let $\eta>0$ be a real number. The conclusion of Theorem 3.4.4 holds for the billiard flow $\left.\left(\mathcal{M}, \mu,\left(Y_{t}\right)_{t}\right)\right)=\left(Q_{1}, \mu_{1},\left(S_{t}\right)_{t}\right)$ in the domain $Q$ and for a function $f: \mathbf{R}^{d} \times \mathcal{T}^{1} Q \rightarrow \mathbf{R}^{d} C_{b}^{2,0}$ such that $f$ is uniformly Hölder continuous of order $\eta$ in the second variable.

\section{Optimality of the estimations - Degenerated CASE}

We fix a real number $T_{0}>0$. We are interested in the problem of the optimality of the estimations

$$
\sup _{0 \leq t \leq T_{0}}\left\|e_{t}^{\varepsilon}(x, \cdot)\right\|_{1}=O(\sqrt{\varepsilon}) \text { and } \sup _{0 \leq t \leq T_{0}}\left\|E_{t}^{\varepsilon}(x, \cdot)\right\|_{1}=O(\sqrt{\varepsilon})
$$

obtained in the previous sections. We shall see how the study of boundness and regularity of coboundaries gives information about the behavior of $e_{t}^{\varepsilon}(x, \cdot)$ and $E_{t}^{\varepsilon}(x, \cdot)$ when the limit process is degenerated.

\subsection{A general result for a dynamical system}

We consider a dynamical system $(\Omega, \nu, \sigma)$ and a measurable function $F: \mathbf{R}^{d} \times \Omega \rightarrow \mathbf{R}^{d}$ uniformly bounded and uniformly $L_{F}$-Lipschitz continuous in the first variable. We shall suppose that $(\Omega, \nu, \sigma)$ is an invertible system. We consider the processes $\left(e_{t}^{\varepsilon}(x, \cdot)=x_{t}^{\varepsilon}(x, \cdot)-w_{t}(x)\right)_{t}\left(\right.$ for $\left.x \in \mathbf{R}^{d}\right)$ where $\left(x_{t}^{\varepsilon}(x, \cdot)\right)_{t}$ and $\left(w_{t}(x)\right)_{t}$ are given by (1.1.3) with initial conditions $x_{0}^{\varepsilon}(x, \cdot)=w_{0}(x)=x$. We suppose that the following quantity is finite

$$
K_{\tilde{F}}:=\sup _{i, j=1, \ldots, d} \sum_{n=-\infty}^{+\infty}(1+|n|) \cdot \sup _{x, y \in \mathbf{R}^{d}}\left|\mathbf{E}_{\nu}\left[\tilde{F}_{i}(x, \cdot) \tilde{F}_{j}\left(y, \sigma^{n}(\cdot)\right)\right]\right| .
$$


In the following, for any point $x$ in $\mathbf{R}^{d}$ and any $i, j=1, \ldots, d, a_{i, j}(x)$ is the quantity:

$$
a_{i, j}(x):=\sum_{n=-\infty}^{+\infty} \mathbf{E}_{\nu}\left[\tilde{F}_{i}(x, \cdot) \tilde{F}_{j}\left(x, \sigma^{n}(\cdot)\right)\right]
$$

We write $\Sigma^{2}(x)=\left(a_{i, j}(x)\right)_{i, j=1, \ldots, d}$. The following result is an easy consequence of Theorem 2.1.3.

Theorem 5.1.1. Let us suppose Hypothesis 2.1.2 satisfied. Let a point $x \in \mathbf{R}^{d}$ and a real number $s>0$ be given. Then, the following properties are equivalent:

(i) the process $\left(e_{t}^{0}(x, \cdot)\right)_{0 \leq t \leq s}$ is identically null (almost surely);

(ii) the process $\left(v_{t}(x, \cdot)\right)_{0 \leq t \leq s}$ is identically null (almost surely);

(iii) for any $t \in[0 ; s]$, the matrix $\Sigma^{2}\left(w_{t}(x)\right)$ is null.

Corollary. Let $x$ be a point in $\mathbf{R}^{d}$ and $s>0$ be a real number such that the matrix $\Sigma^{2}\left(w_{s}(x)\right)$ is not null, then we have

$$
\liminf _{\varepsilon \rightarrow 0} \sup _{t \in[0 ; s]}\left\|\frac{e_{t}^{\varepsilon}(x, \cdot)}{\sqrt{\varepsilon}}\right\|_{1}>0 .
$$

In the following, we shall consider points $x$ for which there exists a real number $s \in\left[0 ; T_{0}\right]$ such that the matrix $\Sigma^{2}\left(w_{t}(x)\right)$ is null for all $t \in[0 ; s]$. Using Lemma 2.2.2, we first notice that we have:

Lemma 5.1.2. If the quantity $K_{\tilde{F}}$ is finite and if $\Sigma^{2}(x)=0$, then, for any integers $i, j=1, \ldots, d$, we have

$$
\mathbf{E}_{\nu}\left[\left(\int_{t_{0}}^{t_{0}+t} \tilde{F}_{i}\left(x, \sigma^{\lfloor s\rfloor}(\cdot)\right)\right)^{2}\right] \leq 2 K_{\tilde{F}} .
$$

Notation. For any point $x$ in $\mathbf{R}^{d}$, we write $t_{x}:=\sup \left\{s \in\left[0 ; T_{0}\right]: \forall t \in[0 ; s], \Sigma^{2}\left(w_{t}(x)\right)=0\right\}$.

Definition. A point $x$ in $\mathbf{R}^{d}$ is called a singular point for the couple $(F, \sigma)$ if we have $t_{x}>0$. We denote by $\mathcal{S}(F, \sigma)$ the set of these points.

Proposition 5.1.3. We suppose that the quantity $K_{\tilde{F}}$ is finite. Let $x$ be a singular point for $(F, \sigma)$. Then, for any real numbers $s$ and $t$ with $0 \leq s \leq t \leq t_{x}$, we have

$$
\left\|v_{t}^{\varepsilon}(x, \cdot)-v_{s}^{\varepsilon}(x, \cdot)\right\|_{2}^{2} \leq C_{0}^{\prime}\left(K_{\tilde{F}}+\|\tilde{F}\|_{\infty}^{2}+L_{\tilde{F}}\|\bar{F}\|_{\infty}\|\tilde{F}\|_{\infty}\right) \varepsilon^{\frac{1}{3}} \sqrt{t-s} .
$$

Proof. We prove this result as we proved Proposition 2.2.3 with $n_{\varepsilon}:=\left\lfloor\varepsilon^{-\frac{2}{3}} \sqrt{t-s}\right\rfloor$.

Corollary. If the quantity $K_{\tilde{F}}$ is finite and if the set $\mathcal{S}(F, \sigma)$ of singular points is non empty, then we have $\sup _{x \in \mathcal{S}(F, \sigma)} \sup _{0 \leq t \leq t_{x}}\left\|e_{t}^{\varepsilon}(x, \cdot)\right\|_{2} \leq O\left(\varepsilon^{\frac{2}{3}}\right)$.

Proof. We use the previous proposition and Remark 2.2.1.

We recall the following classical result:

Lemma 5.1.4. Let $\psi: \Omega \rightarrow \mathbf{R}^{d}$ be a measurable function $\nu$-center, the coordinates of which are denoted by $\psi_{1}, \ldots, \psi_{d}$. If, for any $i, j=1, \ldots, d$, we have $\sum_{k \in \mathbf{Z}}|k| \cdot\left|\mathbf{E}_{\nu}\left[\psi_{i} \cdot \psi_{j} \circ \sigma^{k}\right]\right|<+\infty$, then the following quantity is well defined: $a_{i, j}:=\sum_{k \in \mathbf{Z}} \mathbf{E}_{\nu}\left[\psi_{i} \cdot \psi_{j} \circ \sigma^{k}\right]$ and if, moreover, we have $a_{i, j}=0$ for any $i, j=1, \ldots, d$, then $\psi$ is a coboundary in $L^{2}(\Omega, \nu)$ for $\sigma$, i.e. there exists a square integrable function $H: \Omega \mapsto \mathbf{R}^{d}$ satisfying $\psi=H \circ \sigma-H$ in $L^{2}(\Omega, \nu)$.

If $x$ is in the set $\mathcal{S}(F, \sigma)$ of singular points, then, according to the foregoing, for any $t \in\left[0 ; t_{x}\right]$, there exists a function $K_{t} \in L^{2}(\Omega, \nu)$ satisfying:

$$
\tilde{F}\left(w_{t}(x), \cdot\right)=K_{t}(\sigma(\cdot))-K_{t}(\cdot) \text {, a.e. }
$$


To establish controls, it is useful to have an equality everywhere instead of an equality almost everywhere and to have a regular function $H$ such that $K_{t}(\cdot)=H\left(w_{t}(x), \cdot\right)$. We shall be able to establish the existence of such a function $H$ for different examples. Then, we shall apply the following result, obtained according to Remark 2.2.1.

Theorem 5.1.5. Let $x \in \mathbf{R}^{d}$ be a singular point for $(F, \sigma)$. If there exists a function $H: \mathbf{R}^{d} \times \Omega \rightarrow \mathbf{R}^{d}$ measurable, uniformly bounded and $L_{H}$-uniformly Lipschitz continuous in the first variable such that, for any real number $t \in\left[0 ; t_{x}\right]$, we have $F\left(w_{t}(x), \cdot\right)=H\left(w_{t}(x), \sigma(\cdot)\right)-H\left(w_{t}(x), \cdot\right)$, then we have

$$
\sup _{0 \leq t \leq t_{x}}\left\|e_{t}^{\varepsilon}(x, \cdot)\right\|_{\infty} \leq \varepsilon\left(1+L_{F} t_{x} e^{L_{F} t_{x}}\right)\left(2\|H\|_{\infty}+t_{x} L_{H}\|\bar{F}\|_{\infty}\right) .
$$

\subsection{A general result for a suspension flow}

We suppose that $\left(\mathcal{M}, \mu,\left(Y_{t}\right)_{t}\right)$ is a suspension flow defined over an invertible probabilised dynamical system $(\Omega, \nu, \sigma)$ by a measurable function $\tau: \Omega \rightarrow] 0 ;+\infty\left[\right.$ satisfying $C^{-1} \leq \tau \leq C$ for some constant $C>0$ and that the function $f: \mathbf{R}^{d} \times \mathcal{M} \rightarrow \mathbf{R}^{d}$ is in $\mathcal{C}_{b}^{(1)}$. We consider the processes $\left(E_{t}^{\varepsilon}(x, \cdot)=X_{t}^{\varepsilon}(x, \cdot)-W_{t}(x)\right)_{t}($ for $\left.x \in \mathbf{R}^{d}\right)$ where $\left(X_{t}^{\varepsilon}(x, \cdot)\right)_{t}$ and $\left(W_{t}(x)\right)_{t}$ are given by equations (1.1.1) and (1.1.2) with the initial conditions $X_{0}^{\varepsilon}(x, \cdot)=W_{0}(x)=x$. We suppose that the following quantity is finite:

$$
K_{\Psi}:=\sup _{i, j=1, \ldots, d} \sum_{n=-\infty}^{+\infty}(1+|n|) \cdot \sup _{x, y \in \mathbf{R}^{d}}\left|\mathbf{E}_{\nu}\left[\Psi_{i}(x, \cdot) \Psi_{j}\left(y, \sigma^{n}(\cdot)\right)\right]\right|,
$$

for the function $\Psi: \mathbf{R}^{d} \times \Omega \rightarrow \mathbf{R}^{d}$ defined by $\Psi(x, \omega):=\int_{0}^{\tau(\omega)} \tilde{f}(x,(\omega, u)) \mathrm{d} u$. For any point $x$ in $\mathbf{R}^{d}$, we denote by $\tilde{\Sigma}^{2}(x)$ the $d \times d$ symmetrical positive matrix, the coefficients of which are the $A_{i, j}(x)$ defined by

$$
A_{i, j}(x):=\lim _{t \rightarrow+\infty} \frac{1}{t} \int_{0}^{t} \int_{0}^{t} \mathbf{E}_{\nu}\left[\Psi_{i}\left(x, \sigma^{\lfloor s\rfloor}(\cdot)\right) . \Psi_{j}\left(x, \sigma^{\lfloor u\rfloor}(\cdot)\right)\right] \mathrm{d} s \mathrm{~d} u .
$$

Proposition 5.2.1. We suppose that Hypothesis 3.1 .1 is satisfied. Let $x$ be a point in $\mathbf{R}^{d}$ and $s>0$ be a real number. Then, the following properties are equivalent:

(i) the process $\left(E_{t}^{0}(x, \cdot)\right)_{0 \leq t \leq s}$ is identically null (almost surely);

(ii) the process $\left(\tilde{V}_{t}^{0}(x, \cdot)\right)_{0 \leq t \leq s}$ is identically null (almost surely);

(iii) for any $t \in[0 ; s]$, the matrix $\tilde{\Sigma}^{2}\left(W_{t}(x)\right)$ is null.

For any $x$ in $\mathbf{R}^{d}$, we define $t_{x}^{\prime}$ by: $t_{x}^{\prime}:=\min \left\{s \in\left[0 ; T_{0}\right]: \forall t \in[0 ; s], \tilde{\Sigma}^{2}\left(W_{t}(x)\right)=0\right\}$.

Definition. A point $x$ in $\mathbf{R}^{d}$ is called a singular point for the couple $\left(f,\left(Y_{t}\right)_{t}\right)$ if we have $t_{x}^{\prime}>0$. The set of these points is written $\mathcal{S}\left(f,\left(Y_{t}\right)_{t}\right)$.

Let $x$ be a singular point for $\left(f,\left(Y_{t}\right)_{t}\right)$. According to the foregoing, for any real number $t \in\left[0 ; t_{x}^{\prime}\right]$, the function $\Psi\left(W_{t}(x), \cdot\right)$ is a coboundary in $L^{2}(\Omega, \nu)$. As in the case of a dynamical system, we shall establish results of regularity for coboundaries allowing us to use the following result:

Theorem 5.2.2. Let $x \in \mathbf{R}^{d}$ be a singular point for $\left(f,\left(Y_{t}\right)_{t}\right)$. If there exists a function $H: \mathbf{R}^{d} \times \Omega \rightarrow \mathbf{R}^{d}$ of class $C_{b}^{1, *}$ such that, for any real number $t \in\left[0 ; t_{x}^{\prime}\right]$ and any $\omega$ in $\Omega$, we have

$$
\Psi\left(W_{t}(x), \omega\right)=H\left(W_{t}(x), \sigma(\omega)\right)-H\left(W_{t}(x), \omega\right),
$$

then we have

$$
\sup _{0 \leq t \leq t_{x}^{\prime}}\left\|E_{t}^{\varepsilon}(x, \cdot)\right\|_{\infty}=\varepsilon\left(1+L_{f} t_{x}^{\prime} \mathrm{e}^{L_{f} t_{x}^{\prime}}\right)\left(2\left(\|H\|_{\infty}+\max \tau\|\tilde{f}\|_{\infty}\right)+t_{x}^{\prime}\left(\left\|D_{1} H\right\|_{\infty}+\max \tau \cdot\left\|D_{1} f\right\|_{\infty}\right)\|\bar{f}\|_{\infty}\right) .
$$


The proof of this result is based on the two following lemmas:

Lemma 5.2.3. Let $\psi: \mathcal{M} \mapsto \mathbf{R}^{d}$ be a measurable function such that, for any $\omega$ in $\Omega$, the function $u \mapsto \psi(\omega, u)$ is continuous on $] 0 ; \tau(\omega)$ [ and such that the following limits do exist: $\lim _{u \rightarrow 0+} \psi(\omega, u)$ and $\lim _{u \rightarrow \tau(\omega)-} \psi(\omega, u)$. Then, the following properties are equivalent:

(i) there exists a measurable function $h$ continuous in the direction of the flow (i.e. such that, for any $y \in \mathcal{M}$, $u \mapsto h\left(Y_{u}(y)\right)$ is continuous) and such that $\psi(\omega, u)=\left(\frac{\mathrm{d}}{\mathrm{d} s} h\left(Y_{s}(\omega, u)\right)\right)_{\mid s=0}$, for any $\left.u \in\right] 0, \tau(\omega)[;$

(ii) there exists a measurable function $H$ such that $\Psi=H \circ \sigma-H$, with $\Psi(\omega):=\int_{0}^{\tau(\omega)} \psi(\omega, u) \mathrm{d} u$; and, when these properties are satisfied, we have $h(\omega, s)=H(\omega)+\int_{0}^{s} \psi(\omega, u) \mathrm{d} u$.

Proof of the theorem. The function $h$ defined by $h(x,(\omega, s)):=H(x, \omega)+\int_{0}^{s} \tilde{f}(x,(\omega, u)) \mathrm{d} u$ satisfies the hypotheses of the following lemma and we have $D_{1} h(x,(\omega, s))=D_{1} H(x, \omega)+\int_{0}^{s} D_{1} \tilde{f}(x,(\omega, u)) \mathrm{d} u$.

Lemma 5.2.4. Let $x \in \mathbf{R}^{d}$ be a singular point. We suppose that there exists a function $h: \mathbf{R}^{d} \times \mathcal{M} \mapsto \mathbf{R}^{d} C_{b}^{1, *}$ continuous in the direction of the flow (i.e. such that, for any $(x, y) \in \mathbf{R}^{d} \times \mathcal{M}, u \mapsto h\left(x, Y_{u}(y)\right)$ is continuous) and such that, for any real number $t \in\left[0 ; t_{x}^{\prime}\right]$, we have $\tilde{f}\left(W_{t}(x), \cdot\right)=\frac{\mathrm{d} h}{\mathrm{~d} s}\left(W_{t}(x), Y_{s}(\cdot)\right)_{\mid s=0}($ for any $(s, y)$ such that $\left.Y_{s}(y) \notin \Omega \times\{0\}\right)$ and that, for any $y \in \mathcal{M},(x, s) \mapsto D_{1} h\left(x, Y_{s}(y)\right)$ is continuous. Then, for any $y \in \mathcal{M}$, we have

$$
\sup _{0 \leq t \leq t_{x}^{\prime}}\left|X_{t}^{\varepsilon}(x, y)-W_{t}(x)\right| \leq \varepsilon\left(1+L_{f} t_{x}^{\prime} \mathrm{e}^{L_{f} t_{x}^{\prime}}\right)\left(2\|h\|_{\infty}+t_{x}^{\prime}\left\|D_{1} h\right\|_{\infty}\|\bar{f}\|_{\infty}\right)
$$

Proof. Let $y \in \mathcal{M}$ be fixed. We have, for any real number $s \in\left[0 ; t_{x}^{\prime}\right]$ and any $\varepsilon>0$ such that $Y_{\frac{s}{\varepsilon}}(y) \notin \Omega \times\{0\}$,

$$
\frac{\mathrm{d}}{\mathrm{d} s} h\left(W_{s}(x), Y_{\frac{s}{\varepsilon}}(y)\right)=D_{1} h\left(W_{s}(x), Y_{\frac{s}{\varepsilon}}(y)\right) \frac{\mathrm{d}}{\mathrm{d} s} W_{s}(x)+\frac{1}{\varepsilon} \tilde{f}\left(W_{s}(x), Y_{\frac{s}{\varepsilon}}(y)\right)
$$

Therefore, we have

$$
\begin{aligned}
\int_{0}^{t} \tilde{f}\left(W_{s}(x), Y_{\frac{s}{\varepsilon}}(y)\right) \mathrm{d} s & =\varepsilon \int_{0}^{t} \frac{\mathrm{d}}{\mathrm{d} s} h\left(W_{s}(x), Y_{\frac{s}{\varepsilon}}(y)\right) \mathrm{d} s-\varepsilon \int_{0}^{t} D_{1} h\left(W_{s}(x), Y_{\frac{s}{\varepsilon}}(y)\right) \frac{\mathrm{d}}{\mathrm{d} s} W_{s}(x) \mathrm{d} s \\
& =\varepsilon\left(h\left(W_{t}(x), Y_{\frac{t}{\varepsilon}}(y)\right)-h(x, y)-\int_{0}^{t} D_{1} h\left(W_{s}(x), Y_{\frac{s}{\varepsilon}}(y)\right) \frac{\mathrm{d}}{\mathrm{d} s} W_{s}(x) \mathrm{d} s\right)
\end{aligned}
$$

We conclude by Gronwall lemma, as in Remark 2.2.1.

\subsection{Examples}

We mention the fact that Theorems 5.1.5 and 5.2.2 can be applied to subshifts of finite type and to some dynamical systems for which the transfer operator is quasicompact on some convenient functional normed space $(V,\|\cdot\|)(c f .[14])$. For each one of these two examples, the idea is to consider the function $\Psi=\tilde{F}$ (for the dynamical system) or $\Psi(x, \omega):=\int_{0}^{\tau(\omega)} \tilde{f}(x,(\omega, s)) \mathrm{d} s$ (for a suspension flow over one of these dynamical systems), to get the following decomposition (using operator methods):

$$
\Psi(x, \omega)=G(x, \omega)+H(x, \sigma(\omega))-H(x, \omega),
$$

where $G(x, \cdot)$ is a measurable function generating a sequence of reversed martingale differences, i.e.

$$
\mathbf{E}_{\nu}\left[G(x, \cdot) \mid G\left(x, \sigma^{k}(\cdot)\right), k \geq 1\right]=0
$$


Then we show that we can choose $G$ and $H$ regular and establish an equality for the partial derivatives of $\Psi$, obtained from equation (5.3.1) by derivation:

$$
\frac{\partial}{\partial x_{i}} \Psi(x, \omega)=\frac{\partial}{\partial x_{i}} G(x, \omega)+\frac{\partial}{\partial x_{i}} H(x, \sigma(\omega))-\frac{\partial}{\partial x_{i}} H(x, \omega) .
$$

In these two cases, under suitable conditions of regularity for $F$ and $D_{1} F$, we get:

$$
\sup _{x \in \mathcal{S}(F, \sigma)} \sup _{0 \leq t \leq t_{x}}\left\|e_{t}^{\varepsilon}(x, \cdot)\right\|_{\infty}=O(\varepsilon) \text { and } \sup _{x \in \mathcal{S}\left(f,\left(Y_{t}\right)_{t}\right)} \sup _{0 \leq t \leq t_{x}^{\prime}}\left\|E_{t}^{\varepsilon}(x, \cdot)\right\|_{\infty}=O(\varepsilon)
$$

Here, we are interested in the examples of ergodic algebraic toral automorphisms and of dispersive billiards. For these examples, we shall get weaker results. In the case of ergodic toral automorphisms, we shall establish such results only when $G$ is identically null. For Sinai dispersive billiard, we shall get results in $L^{p}$.

\subsubsection{Ergodic algebraic toral automorphisms}

Let $\sigma=T$ be an ergodic algebraic automorphism of the torus $\Omega=\mathbf{T}^{n}$, endowed with the Haar measure $\nu$.

Theorem 5.3.1. If $F$ is $C_{b}^{1,0}$ and if, for any $x \in \mathbf{R}^{d}$ and any integer $i=1, \ldots, d$, the functions $\tilde{F}(x, \cdot)$ and $\frac{\partial}{\partial x_{i}} \tilde{F}(x, \cdot)$ are $n$-times differentiable, the $n^{\text {th }}$ differential of which is uniformly Hölder continuous of order $\eta$ and if $\Sigma^{2}(x)=0$, for any $x$ in $\mathbf{R}^{d}$, then we have

$$
\sup _{x \in \mathbf{R}^{d}} \sup _{0 \leq t \leq T_{0}}\left\|e_{t}^{\varepsilon}(x, \cdot)\right\|_{\infty}=O(\varepsilon)
$$

We follow here the proof of the following result given in [4]. Our proof consists essentially in showing that the inequalities established in [4] are uniform in $x$.

Proposition 5.3.2. Let $f: \mathbf{T}^{n} \mapsto \mathbf{R}$ be a function, $n$-times differentiable, the $n^{\text {th }}$ differential of which is Hölder continuous. If $f$ is a measurable coboundary, then it is a coboundary in the set of the Hölder continuous functions.

The proof of this proposition is based on the two following lemmas. We shall use the first without any proof and we shall rewrite the proof of the second one to establish Theorem 5.3.1.

Lemma 5.3.3 (cf. [1]). Let $\alpha>0$ be a real number. There exists a constant $K_{0}>0$ such that, for any non zero integer vector $k \in \mathbf{Z}^{n}$ and any function $g: \mathbf{T}^{n} \rightarrow \mathbf{R}^{d}$-times differentiable, the $n^{\text {th }}$ differential of which is Hölder continuous of order $\alpha$, we have

$$
\left|c_{k}(g)\right| \leq K_{0} C_{D^{n} g}^{(\alpha)}\|k\|^{\prime-n-\alpha}
$$

Lemma 5.3.4. Let $l>0$ and $\varepsilon>0$ be two real numbers. There exists a constant $K=K_{l, \varepsilon}>0$ such that if the Fourier coefficients of the function $f: \mathbf{T}^{n} \rightarrow \mathbf{R}$ satisfy, for any non zero integer $k$,

$$
\left.\left|c_{k}(f)\right| \leq K_{f}\|k\|^{-l} \quad \text { with } \quad K_{f} \in\right] 0 ;+\infty[
$$

and if there exists a function $h$ in $L^{2}\left(\mathbf{T}^{n}\right)$ satisfying the coboundary equation $f=h-h \circ T$, then the Fourier coefficients of h satisfy

$$
\left|c_{k}(h)\right| \leq K \cdot K_{f}\|k\|^{\varepsilon-l}
$$

Proof of the theorem. We have $\tilde{F}(x, \cdot)=H(x, T(\cdot))-H(x, \cdot)$ with $H(x, \cdot)$ in $L^{2} \nu$-centered. Let us show that $H$ is $C_{b}^{1,0}$. We follow the proof of Proposition 5.3.2 and of Lemma 5.3.4 given in [4]. 
1. For any integer vector $k \in \mathbf{Z}^{n}$, we denote by $c_{k}(x)$ (resp. $\left.d_{k}(x)\right)$ the $k^{\text {th }}$ Fourier coefficient of $\tilde{F}(x, \cdot)$ (resp. $H(x, \cdot))$. Following [4], we observe that we have

$$
d_{k}(x)=\sum_{j \geq 0} c_{S^{-j} k}(x)=-\sum_{j \geq 1} c_{S^{j} k}(x)
$$

2. We denote by $c_{k, i}^{\prime}(x)$ the $k^{\text {th }}$ Fourier coefficient of $\frac{\partial}{\partial x_{i}} \tilde{F}(x, \cdot)$. Then, for any $x \in \mathbf{R}^{d}$ and any $i=1, \ldots, d$, we have: $c_{k, i}^{\prime}(x)=\frac{\partial}{\partial x_{i}} c_{k}(x)$.

3. For each $k$, the serie $\sum_{j \geq 0} c_{S^{-j} k, i}^{\prime}(x)$ is normally convergent.

Since $\frac{\partial}{\partial x_{i}} \tilde{F}(x, \cdot)$ is $n$-times differentiable, the $n^{\text {th }}$ differential of which is Hölder continuous of order $\alpha$, we have

$$
\left|c_{k, i}^{\prime}(x)\right| \leq K_{0} C_{D^{n} \frac{\partial}{\partial x_{i}}}^{(\alpha)} \tilde{F}(x, \cdot) \mid\|k\|^{-n-\alpha},
$$

according to Lemma 5.3.3. Thus, we have

$$
\sum_{j \geq 0}\left|c_{S^{-j} k, i}^{\prime}(x)\right| \leq K_{0} \sup _{x^{\prime} \in \mathbf{R}^{d}} \sup _{i=1, \ldots, d} C_{D^{n} \frac{\partial}{\partial x_{i}} \tilde{F}\left(x^{\prime}, \cdot\right)}^{(\alpha)} \sum_{j \geq 0}\left\|S^{-j} k\right\|^{\prime-n-\alpha} .
$$

Now, we have $\left\|S^{-j} k\right\|^{\prime} \geq\left\|S^{-j}\left(k_{s}\right)\right\|^{\prime}$. We denote $r:=\max \left(\rho\left(S_{\mid E_{s}}\right), \rho\left(\left(S^{-1}\right)_{\mid E_{u}}\right)\right)$. Let us choose a real number $r_{1}$ satisfying $r<r_{1}<1$. Then, there exists a constant $C_{1}>0$ such that, for any $x \in E_{s}$, any $y \in E_{u}$ and any integer $j \geq 0$, we have

$$
\left\|S^{j} x\right\|^{\prime} \leq C_{1} r_{1}^{j}\|x\|^{\prime} \text { and }\left\|S^{-j} y\right\|^{\prime} \leq C_{1} r_{1}^{j}\|y\|^{\prime} .
$$

So, we have

$$
\left\|S^{-j} k\right\|^{\prime} \geq\left\|S^{-j}\left(k_{s}\right)\right\|^{\prime} \geq \frac{r_{1}^{-j}}{C_{1}}\left\|k_{s}\right\|^{\prime} .
$$

4. We denote $d_{k, i}^{\prime}(x):=\sum_{j \geq 0} c_{S^{-j} k, i}^{\prime}(x)$. According to the foregoing, $d_{k, i}^{\prime}(x)$ is well defined, $d_{k}$ is differentiable and we have $\frac{\partial}{\partial x_{i}} d_{k}(x)=d_{k, i}^{\prime}(x)$. In the same way, we get $d_{k, i}^{\prime}(x)=\frac{\partial}{\partial x_{i}} d_{k}(x)=-\sum_{j \geq 1} c_{S^{j} k, i}^{\prime}(x)$. We show that the series of functions $\sum_{k} d_{k, i}^{\prime}(x)$ are normally convergent. To see this, we adapt the proof of Lemma 5.3.4 proposed by Le Borgne in [4]. Since $\frac{\partial}{\partial x_{i}} \tilde{F}(x, \cdot)$ is $n$-times differentiable with $n^{\text {th }}$ differential Hölder continuous of order $\alpha$, we obtain the same kind of inequalities for $c_{k, i}^{\prime}(x)$ as for the coefficients $c_{k}(x)$. Let $k \in \mathbf{Z}^{n}$ be a non zero integer vector.

- If we have $\|k\|^{\prime}=\left\|k_{u}\right\|^{\prime}$, then we have

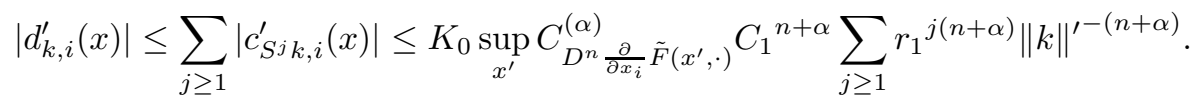

- If we have $\|k\|^{\prime}=\left\|k_{s}\right\|^{\prime}$, then we have

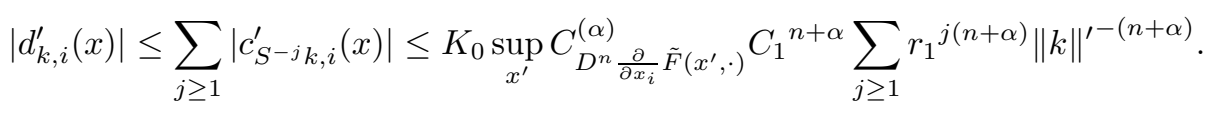

- If we have $\|k\|^{\prime}=\left\|k_{e}\right\|^{\prime}$, then we have $\left\|k_{u}\right\|^{\prime} \geq \frac{K_{(e, s)}^{\prime}}{\|k\|^{\prime n}}$ and therefore

$$
\left\|S^{j} k\right\|^{\prime} \geq\left\|S^{j} k_{u}\right\|^{\prime} \geq \frac{1}{C_{1}} r_{1}^{-j}\left\|k_{u}\right\|^{\prime} \geq \frac{1}{C_{1}} r_{1}{ }^{-j} K_{(e, s)}^{\prime}\|k\|^{\prime-n} .
$$


Let us denote $j(k):=\left\lceil\frac{2(n+1) \ln \left(\|k\|^{\prime}\right)}{-\ln \left(r_{1}\right)}\right\rceil$. If we have $j \geq j(k)$, then we have $r_{1}{ }^{-j}\|k\|^{\prime-n} \geq r_{1}^{-\frac{j}{2}}\|k\|^{\prime}$ and therefore

$$
\left\|S^{j} k\right\|^{\prime} \geq \frac{1}{C_{1}} K_{(e, s)}^{\prime} r_{1}^{-\frac{j}{2}}\|k\|^{\prime} .
$$

On the other hand, there exists a real number $R_{1}>0$ such that, for any $x$ in $E_{e}$ and any integer $j \neq 0$, we have

$$
\left\|S^{j} x\right\|^{\prime} \leq R_{1}|j|^{n} \cdot\|x\|^{\prime} .
$$

If we have $1 \leq j \leq j(k)$, then we have

$$
\left\|S^{j} k\right\|^{\prime} \geq\left\|S^{j} k_{e}\right\|^{\prime} \geq \frac{1}{R_{1}} j^{-n}\left\|k_{e}\right\|^{\prime} \geq \frac{1}{R_{1}}(j(k))^{-n}\|k\|^{\prime} .
$$

So, we have

$$
\begin{aligned}
\left|d_{k, i}^{\prime}(x)\right| \leq & \sum_{j \geq 1}\left|c_{S^{j} k, i}^{\prime}(x)\right| \\
\leq & K_{0} \sup _{x^{\prime}} C_{D^{n} \frac{\partial}{\partial x_{i}} \tilde{F}\left(x^{\prime}, \cdot\right)}^{(\alpha)} \sum_{j \geq 1}\left\|S^{j} k\right\|^{\prime-(n+\alpha)} \\
\leq & K_{0} \sup _{x^{\prime}} C_{D^{n} \frac{\partial}{\partial x_{i}} \tilde{F}\left(x^{\prime}, \cdot\right)}^{(\alpha)}\left(R_{1}^{n+\alpha} \sum_{j=1}^{j(k)}(j(k))^{n(n+\alpha)}\|k\|^{\prime-(n+\alpha)}\right. \\
& \left.+\sum_{j \geq j(k)} \frac{C_{1}{ }^{n+\alpha}}{K_{(e, s)}^{\prime}{ }^{n+\alpha}} r_{1}^{\frac{j(n+\alpha)}{2}}\|k\|^{\prime-(n+\alpha)}\right) \\
\leq & K_{1} \sup _{x^{\prime}} C_{D^{n} \frac{\partial}{\partial x_{i}} \tilde{F}\left(x^{\prime}, \cdot\right)}^{(\alpha)}\left((j(k))^{n(n+\alpha)+1}\|k\|^{-(n+\alpha)}+\sum_{j \geq j(k)} r_{1}^{\frac{j(n+\alpha)}{2}}\|k\|^{\prime-(n+\alpha)}\right) \\
\leq & K_{2} \sup _{x^{\prime}} C_{D^{n} \frac{\partial}{\partial x_{i}} \tilde{F}\left(x^{\prime}, \cdot\right)}^{(\alpha)}\left(\ln \left(\|k\|^{\prime}\right)\right)^{n(n+\alpha)+1}\|k\|^{\prime-(n+\alpha)},
\end{aligned}
$$

for some constants $K_{1}>0$ and $K_{2}>0$.

5. We conclude that $H$ is differentiable in the first variable and that its partial derivatives satisfy

$$
\frac{\partial}{\partial x_{i}} H(x, \cdot)=\sum_{k} d_{k, i}^{\prime}(x) \mathrm{e}^{2 i \pi\langle k \cdot \cdot\rangle}
$$

therefore are continuous and uniformly bounded.

We can show the following result in the same way.

Theorem 5.3.5. We suppose that $\left(\mathcal{M}, \mu,\left(Y_{t}\right)_{t}\right)$ is the suspension flow defined over $\left(\mathbf{T}^{n}, \nu, T\right)$ by a measurable function $\left.\tau: \mathbf{T}^{n} \rightarrow\right] 0 ;+\infty\left[\right.$ satisfying $C^{-1} \leq \tau \leq C$ for some constant $C>0$. If $f$ is in $\mathcal{C}_{b}^{(1)}$ and if, for all $x \in \mathbf{R}^{d}$ and all integer $i=1, \ldots, d$, the functions $\Psi(x, \cdot):=\int_{0}^{\tau(\cdot)} \tilde{f}(x,(\cdot, u)) \mathrm{d} u$ and $\frac{\partial}{\partial x_{i}} \Psi(x, \cdot)$ are $n$-times differentiable, with $n^{\text {th }}$ differential uniformly Hölder continuous of order $\eta$ and if $\tilde{\Sigma}^{2}(x)=0$, for all $x$ in $\mathbf{R}^{d}$, then we have

$$
\sup _{x \in \mathbf{R}^{d}} \sup _{0 \leq t \leq T_{0}}\left\|E_{t}^{\varepsilon}(x, \cdot)\right\|_{\infty}=O(\varepsilon) .
$$




\subsubsection{Sinai dispersive billiard with finite horizon}

Following [18], we can prove the following result (cf. Th. 4.3.2 in [14]). A sketch of its proof is given in Appendix $\mathrm{C}$ of the present paper.

Theorem 5.3.6. Let $\eta>0$ be a real number and $m_{0}$ be an integer. There exists an invertible extension of $(M, \nu, T)$ given by $\dot{\pi}:(\dot{M}, \dot{\nu}, \dot{T}) \rightarrow(M, \nu, T)$ and a sub- $\sigma$-algebra $\dot{B}$ for $\dot{M}$ such that, for any integer $p \geq 1$, there exists some constants $\tilde{D}_{\eta, m_{0}, p}>0$ and $\left.\tilde{\theta}_{\eta, m_{0}, p} \in\right] 0 ; 1\left[\right.$ such that, for any $\nu$-centered function $f$ in $\mathcal{H}_{\eta, m_{0}}$, and any integer $j \geq 0$, we have:

$$
\left\|\mathbf{E}_{\dot{\nu}}\left[f \circ \dot{\pi} \mid \dot{T}^{-j}(\dot{\mathcal{B}})\right]\right\|_{L^{p}(\dot{M}, \dot{\nu})}+\left\|\mathbf{E}_{\dot{\nu}}\left[f \circ \dot{\pi} \mid \dot{T}^{j}(\dot{\mathcal{B}})\right]-f \circ \dot{\pi}\right\|_{L^{p}(\dot{M}, \dot{\nu})} \leq \tilde{D}_{\eta, m_{0}, p}\|f\|_{\eta, m_{0}}\left(\tilde{\theta}_{\eta, m_{0}, p}\right)^{j} .
$$

Therefore, according to a result of Gordin [8], for any $\nu$-centered function $f$ in $\mathcal{H}_{\eta, m_{0}}$, there exist two real valued functions $g$ and $h$ defined on $\dot{M}$ and belonging to all $L^{p}$ such that we have

$$
f \circ \dot{\pi}=g+h-h \circ \dot{T}, \quad \dot{\nu}-a . e .
$$

where function $g$ generates a sequence of reversed martingale differences $\left(g \circ \dot{T}^{n}\right)_{n}$. Moreover, $h$ is given by the following formula:

$$
h=h(f):=\sum_{j \geq 0}\left(f \circ \dot{\pi} \circ \dot{T}^{j}-\mathbf{E}_{\dot{\nu}}\left[f \circ \dot{\pi} \mid \dot{T}^{j}(\dot{\mathcal{B}})\right] \circ \dot{T}^{j}\right)-\sum_{j \geq 1} \mathbf{E}_{\dot{\nu}}\left[f \circ \dot{\pi} \mid \dot{T}^{-j}(\dot{\mathcal{B}})\right] \circ \dot{T}^{-j} .
$$

Theorem 5.3.7. Let $(\Omega, \nu, \sigma)$ be the billiard system $(M, \nu, T)$ in $Q$. Let $\eta>0$ be a real number, $m_{0}$ be an integer and $F: \mathbf{R}^{d} \times M \rightarrow \mathbf{R}^{d}$ be a function $C_{b}^{1, *}$, with $\tilde{F}_{i}(x, \cdot)$ and $\frac{\partial}{\partial x_{j}} \tilde{F}_{i}(x, \cdot)$ uniformly in $\mathcal{H}_{\eta, m_{0}}$, i.e.

$$
\sup _{x, i}\left\|\tilde{F}_{i}(x, \cdot)\right\|_{\eta, m_{0}}+\sup _{x, i, j}\left\|\frac{\partial \tilde{F}_{i}}{\partial x_{j}}(x, \cdot)\right\|_{\eta, m_{0}}<+\infty
$$

If the set $\mathcal{S}(F, T)$ of singular points for $(F, T)$ is non empty, then, for any integer $p \geq 1$, we have:

$$
\sup _{x \in \mathcal{S}(F, T)} \sup _{t \in\left[0 ; t_{x}\right]}\left\|e_{t}^{\varepsilon}(x, \cdot)\right\|_{L^{p}(\nu)}=O(\varepsilon)
$$

Sketch of the proof. Let an integer $p \geq 1$ be fixed. We shall apply the foregoing to $f=\tilde{F}(x, \cdot)$ for each $x$ in $\mathbf{R}^{d}$. We consider the function $\dot{H}: \mathbf{R}^{d} \times \dot{M} \rightarrow \mathbf{R}^{d}$ defined by $\dot{H}(x, \dot{\omega}):=h(\tilde{F}(x, \cdot))(\dot{\omega})$. We notice that, each time we have $\Sigma^{2}\left(x^{\prime}\right)=0$, we have $\tilde{F}\left(x^{\prime}, \dot{\pi}(\cdot)\right)=\dot{H}\left(x^{\prime}, \cdot\right)-\dot{H}\left(x^{\prime}, \dot{T}(\cdot)\right)$. Moreover, we have: $\sup _{x \in \mathbf{R}^{d}}\|\dot{H}(x, \cdot)\|_{p}<+\infty$ and $\sup _{x, x^{\prime} \in \mathbf{R}^{d}} \frac{\left\|\dot{H}(x, \cdot)-\dot{H}\left(x^{\prime}, \cdot\right)\right\|_{L^{p}(\dot{\nu})}}{\left|x^{\prime}-x\right|}<+\infty$. We conclude as we proved Theorem 5.1.5.

Theorem 5.3.8. Let $\left(\mathcal{M}, \mu,\left(Y_{t}\right)_{t}\right)$ be the billiard flot $\left(Q_{1}, \mu_{1},\left(Y_{t}\right)_{t}\right)$ in $Q$. Let $\eta>0$ be a real number, mo be an integer and $f: \mathbf{R}^{d} \times \mathcal{T}^{1} Q \rightarrow \mathbf{R}^{d}$ be a function $C_{b}^{2,0}$, such that $f_{i}(x, \cdot), \frac{\partial}{\partial x_{j}} f_{i}(x, \cdot)$ and $\frac{\partial^{2}}{\partial x_{j} \partial x_{k}} f_{i}(x, \cdot)$ are uniformly $\eta$-Hölder continuous. If the set $\mathcal{S}\left(f,\left(Y_{t}\right)_{t}\right)$ of singular points for $\left(f,\left(Y_{t}\right)_{t}\right)$ is non empty, then, for any integer $p \geq 1$, we have:

$$
\sup _{x \in \mathcal{S}\left(f,\left(Y_{t}\right)_{t}\right)} \sup _{t \in\left[0 ; t_{x}^{\prime}\right]}\left\|E_{t}^{\varepsilon}(x, \cdot)\right\|_{L^{p}(\mu)}=O(\varepsilon)
$$

Sketch of the proof. Let us write $\Psi(x, \omega):=\int_{0}^{\tau(\omega)} f(x,(\omega, s)) \mathrm{d} s$. Then, $\Psi$ is $C_{b}^{2, *}$. Moreover, functions $\Psi_{i}(x, \cdot)$, $\frac{\partial}{\partial x_{j}} \Psi_{i}(x, \cdot)$ and $\frac{\partial^{2}}{\partial x_{j} \partial x_{k}} \Psi_{i}(x, \cdot)$ are uniformly in $\mathcal{H}_{\eta, 1}$. We consider the functions $\dot{H}: \mathbf{R}^{d} \times \dot{M} \rightarrow \mathbf{R}^{d}$ and 
$\dot{H}_{1}: \mathbf{R}^{d} \times \dot{M} \rightarrow \mathbf{R}^{d}$ defined by $\dot{H}(x, \dot{\omega}):=h(\Psi(x, \cdot))(\dot{\omega})$ and $\dot{H}_{1}(x, \dot{\omega}):=h\left(D_{1} \Psi(x, \cdot)\right)(\dot{\omega})$, respectively. We notice that, each time we have $\tilde{\Sigma}^{2}\left(x^{\prime}\right)=0$, we have

$$
\Psi\left(x^{\prime}, \dot{\pi}(\cdot)\right)=\dot{H}\left(x^{\prime}, \cdot\right)-\dot{H}\left(x^{\prime}, \dot{T}(\cdot)\right) \text { and } D_{1} \Psi\left(x^{\prime}, \dot{\pi}(\cdot)\right)=\dot{H}_{1}\left(x^{\prime}, \cdot\right)-\dot{H}_{1}\left(x^{\prime}, \dot{T}(\cdot)\right) \text {. }
$$

Moreover, we have $D_{1} H=H_{1}$ in $L^{p}$; indeed, we have: $\sup _{x \in \mathbf{R}^{d}} \frac{\left\|\dot{H}(x, \cdot)-\dot{H}\left(x^{\prime}, \cdot\right)-\dot{H}_{1}(x, \cdot) \cdot\left(x^{\prime}-x\right)\right\|_{L^{p}}}{\left|x^{\prime}-x\right|^{2}}<+\infty$. We consider the special flow $\left(\dot{\mathcal{M}}, \dot{\mu},\left(\dot{Y}_{t}\right)_{t}\right)$ defined over $(\dot{M}, \dot{\nu}, \dot{T})$ by the function $\tau \circ \dot{\pi}$. Then, $\left(\dot{\mathcal{M}}, \dot{\mu},\left(\dot{Y}_{t}\right)_{t}\right)$ is an extension of $\left(\mathcal{M}, \mu,\left(Y_{t}\right)_{t}\right)$ by $\dot{\Pi}:(\dot{\omega}, s) \mapsto(\dot{\pi}(\dot{\omega}), s)$. Let us write $\dot{h}(x,(\dot{\omega}, s)):=\dot{H}(x, \dot{\omega})+\int_{0}^{s} \tilde{f}(x,(\dot{\pi}(\dot{\omega}), u)) \mathrm{d} u$ and $\dot{h}_{1}(x,(\dot{\omega}, s)):=\dot{H}_{1}(x, \dot{\omega})+\int_{0}^{s} D_{1} \tilde{f}(x,(\dot{\pi}(\dot{\omega}), u)) \mathrm{d} u$. Let $x$ be in $\mathbf{R}^{d}$. Then, for any $t \in\left[0 ; t_{x}^{\prime}\right]$, we have, in $L^{p}$,

$$
\tilde{f}\left(W_{t}(x), \dot{\Pi}(\cdot)\right)=\frac{\mathrm{d}}{\mathrm{d} s} \dot{h}\left(W_{t}(x), \dot{Y}_{s}(\cdot)\right)_{\mid s=0} \quad \text { and } \quad D_{1} \tilde{f}\left(W_{t}(x), \dot{\Pi}(\cdot)\right)=\frac{\mathrm{d}}{\mathrm{d} s} \dot{h}_{1}\left(W_{t}(x), \dot{Y}_{s}(\cdot)\right)_{\mid s=0} .
$$

Thus, we have, in $L^{p}$,

$$
\frac{\mathrm{d}}{\mathrm{d} s} \dot{h}\left(W_{s}(x), \dot{Y}_{\frac{s}{\varepsilon}}(y)\right)=\dot{h}_{1}\left(W_{s}(x), \dot{Y}_{\frac{s}{\varepsilon}}(y)\right) \bar{f}\left(W_{s}(x)\right)+\frac{1}{\varepsilon} \tilde{f}\left(W_{s}(x), \dot{\Pi}\left(\dot{Y}_{\frac{s}{\varepsilon}}(y)\right)\right),
$$

for any $t \in\left[0 ; t_{x}^{\prime}\right]$. We conclude as in the proof of Theorem 5.2.2.

\section{Appendix A. Construction of Young's tower: Recalls}

In Appendix A, we recall Young's construction for our billiard system. In Appendix B, we give a proof of Proposition 4.2.1 to be complete. This result has already been proved in [15] by the same method. In Appendix C, we give the idea of the proof of Theorem 5.3.6 using the construction recalled in Appendix A.

Let a real number $\eta \in] 0 ; 1[$ be fixed.

\section{Stable and unstable curves}

Hyperbolic properties of $(M, \nu, T)$ (existence and absolute continuity of stable and unstable foliations) are useful to make Young's construction. We recall here some well known results about stable and unstable curves for $(M, \nu, T)$.

Definition. We call curve of $M$ a curve $\gamma$ contained in a connected component of $M$ and which is $C^{1}$ for the parametrisation by $(r, \varphi)$.

For such a curve $\gamma$, we write $l(\gamma):=\int_{\gamma} \sqrt{d r^{2}+d \varphi^{2}}$.

We call stable curve (resp. unstable curve) a curve $\gamma^{s}$ (resp. $\gamma^{u}$ ) contained in $M \backslash R_{-\infty, 0}$ (resp. in $\left.M \backslash R_{0,+\infty}\right)$ and satisfying

$$
\lim _{n \rightarrow+\infty} l\left(T^{n}\left(\gamma^{s}\right)\right)=0 \quad\left(\text { resp. } \quad \lim _{n \rightarrow+\infty} l\left(T^{-n}\left(\gamma^{u}\right)\right)=0\right) .
$$

We recall the following results:

Proposition A.1. There exists a set $\mathcal{N}$ of $M$, exactly $T$-invariant, such that $\nu(\mathcal{N})=1$ and such that any $x \in \mathcal{N}$ is contained in a unique maximal stable curve written $\gamma^{s}(x)$ and in a unique maximal unstable curve written $\gamma^{u}(x)$. 
Proposition A.2. There exist two real numbers $\alpha \in] 0 ; 1\left[\right.$ and $C>0$ such that, for any stable curve $\gamma^{s}$, any unstable curve $\gamma^{u}$ and any integer $n \geq 0$, we have

$$
l\left(T^{n}\left(\gamma^{s}\right)\right) \leq C \alpha^{n} \text { and } l\left(T^{-n}\left(\gamma^{u}\right)\right) \leq C \alpha^{n} .
$$

Moreover, the intersection of a stable curve with an unstable curve contains at most one point.

Following Young [18], we can construct an extension $\left(\tilde{M}_{d}, \tilde{\nu}_{d}, \tilde{T}_{d}\right)$ of $\left(M, \nu, T^{d}\right)$ (for some integer $d \geq 1$ ) and a factor $\left(\hat{M}_{d}, \hat{\nu}_{d}, \hat{T}_{d}\right)$ of $\left(\tilde{M}_{d}, \tilde{\nu}_{d}, \tilde{T}_{d}\right)$ for which the transfer operator has "good" spectral properties on some functional space. The idea of the proof of the strong decorrelation property given in Proposition 4.2.1 is to prove an analogous result for $\left(M, \nu, T^{d}\right)$ using these constructions. We shall establish these results after having briefly recalled the method of construction of these dynamical systems and stressing on the properties that shall be useful for our purpose. We recall the notions of extension and factor.

Definition. Let $\left(\Omega_{0}, \mu_{0}, \theta_{0}\right)$ and $\left(\Omega_{1}, \mu_{1}, \theta_{1}\right)$ be two dynamical systems. The system $\left(\Omega_{1}, \mu_{1}, \theta_{1}\right)$ is said to be an extension of $\left(\Omega_{0}, \mu_{0}, \theta_{0}\right)$ by the map $\pi: \Omega_{1} \rightarrow \Omega_{0}$ if:

- the map $\pi$ is measurable;

- $\mu_{0}$ is the image measure of $\mu_{1}$ by $\pi$, i.e. $\mu_{0}(A)=\mu_{1}\left(\pi^{-1}(A)\right)$ for any measurable subset $A$ of $\Omega_{0}$;

- we have: $\pi \circ \theta_{1}=\theta_{0} \circ \pi$.

We also say that $\left(\Omega_{0}, \mu_{0}, \theta_{0}\right)$ is the factor of $\left(\Omega_{1}, \mu_{1}, \theta_{1}\right)$ by $\pi$.

\section{An extension of $(M, \nu, T)$}

Definition. We call rectangle of $M$ a measurable subset $A$ of $M$ of the following form:

$$
A=\left(\bigcup_{\gamma^{s} \in \Gamma_{A}^{s}} \gamma^{s}\right) \cap\left(\bigcup_{\gamma^{u} \in \Gamma_{A}^{u}} \gamma^{u}\right),
$$

where $\Gamma_{A}^{s}$ is a family of stable curves and $\Gamma_{A}^{u}$ a family of unstable curves and such that $\gamma^{s} \cap \gamma^{u} \neq \emptyset$, for any $\left(\gamma^{s}, \gamma^{u}\right) \in \Gamma_{A}^{s} \times \Gamma_{A}^{u}$.

Let a rectangle $A$ of $M$ be given. We call $s$-sub-rectangle of $A$ a rectangle $B$ of the following form:

$$
B=\left(\bigcup_{\gamma^{s} \in \Gamma_{B}^{s}} \gamma^{s}\right) \cap\left(\bigcup_{\gamma^{u} \in \Gamma_{A}^{u}} \gamma^{u}\right),
$$

with $\Gamma_{B}^{s}$ contained in $\Gamma_{A}^{s}$. We call $u$-sub-rectangle of $A$ a rectangle $C$ of the following form:

$$
C=\left(\bigcup_{\gamma^{s} \in \Gamma_{A}^{s}} \gamma^{s}\right) \cap\left(\bigcup_{\gamma^{u} \in \Gamma_{C}^{u}} \gamma^{u}\right),
$$

with $\Gamma_{C}^{u}$ contained in $\Gamma_{A}^{u}$.

In [18], Young gives the construction of a rectangle $\Lambda=\left(\bigcup_{\gamma^{s} \in \Gamma^{s}} \gamma^{s}\right) \cap\left(\bigcup_{\gamma^{u} \in \Gamma^{u}} \gamma^{u}\right)$ contained in $\mathcal{N}$ (where $\Gamma^{s}$ is a family of stable curves contained in $M \backslash R_{1}$ and $\Gamma^{u}$ a family of unstable curves contained in $M \backslash R_{-1}$ ) endowed with a return time $R(\cdot)$ in $\Lambda$ under the action of $T$ and of a (countable) $\nu$-essential partition $\left\{\Lambda_{i}\right\}_{i \geq 0}$ 
of $\Lambda$ in $s$-sub-rectangles satisfying (in particular) the following:

- $R$ is equal to a constant $r_{i}$ on each $\Lambda_{i}$;

- for any $x \in \Lambda$, we have:

$$
T^{R(x)}\left(\gamma^{s}(x)\right) \subseteq \gamma^{s}\left(T^{R(x)}(x)\right) \quad \text { and } \quad T^{R(x)}\left(\gamma^{u}(x)\right) \supseteq \gamma^{u}\left(T^{R(x)}(x)\right) ;
$$

- for any $i \geq 0, T^{r_{i}}\left(\Lambda_{i}\right)$ is a $u$-sub-rectangle of $\Lambda$;

- $\Lambda_{i}$ is contained in a connected component of $M \backslash R_{-r_{i}, 0}$.

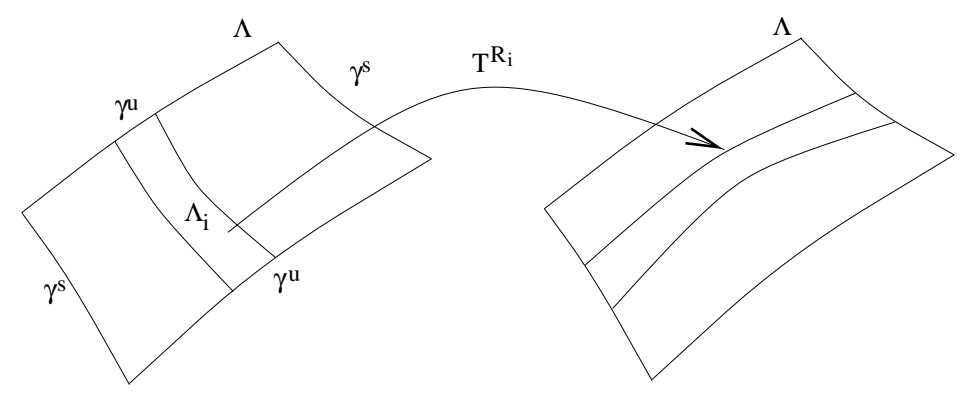

Then, she constructs a Borel probability measure $\tilde{\mu}$ on $\Lambda, T^{R(\cdot)}$-invariant, such that $\mathbf{E}_{\tilde{\mu}}[R]<+\infty$ and such that the "dynamical suspension system" $\left(\tilde{M}_{1}, \tilde{\nu}_{1}, \tilde{T}_{1}\right)$ over $\left(\Lambda, \tilde{\mu}, T^{R(\cdot)}\right)$ defined by the function $R(\cdot)$ as follows is an extension of $(M, \nu, T)$ (by $\tilde{\pi}_{1}: \tilde{M}_{1} \rightarrow M$ given by $\left.\tilde{\pi}_{1}(x, l)=T^{l}(x)\right)$ :

- $\tilde{M}_{1}:=\{(x, l): x \in \Lambda, 0 \leq l \leq R(x)-1\}$;

- $\tilde{T}_{1}(x, l)=(x, l+1)$ if $l<R(x)-1$ and $\tilde{T}_{1}(x, l)=\left(T^{R(x)}(x), 0\right)$ if $l=R(x)-1$;

- $\tilde{\nu}_{1}\left(\bigcup_{l \geq 0} A_{l} \times\{l\}\right)=\frac{\sum_{l \geq 0} \tilde{\mu}\left(A_{l}\right)}{\mathbf{E}_{\tilde{\mu}}[R(\cdot)]}$, where, for each $l, A_{l}$ is a measurable subset of $\{R>l\}$.

\section{A partition}

We define $i_{l}:\{x \in \Lambda: R(x)>l\} \rightarrow \Delta_{l}$ by $i_{l}(x)=(x, l)$. Young gives the construction of a partition $\mathcal{D}=\left\{\Delta_{l, j} ; l \geq 0, j=1, \ldots, j_{l}\right\}$ where $\left\{\Delta_{l, j}\right\}_{j}$ is a finite partition of the $l^{\text {th }}$ "store" $\Delta_{l}:=\left\{\left(x, l^{\prime}\right) \in \tilde{M}_{1} ; l^{\prime}=l\right\}$ satisfying the following properties:

Properties A.3. 1. $j_{0}=1$ and $\Delta_{0,1}=\Delta_{0}=\Lambda \times\{0\}$;

2. each $i_{l}^{-1}\left(\Delta_{l, j}\right)$ is a $s$-sub-rectangle of $\Lambda$, union of $\Lambda_{i}$;

3. for any $l \geq 0,\left\{i_{l+1}{ }^{-1}\left(\Delta_{l+1, j^{\prime}}\right) ; j^{\prime}=1, \ldots, j_{l+1}\right\}$ is a partition of $\{R>l+1\}$ finer than the one induced by $\left\{i_{l}{ }^{-1}\left(\Delta_{l, j^{\prime}}\right) ; j^{\prime}=1, \ldots, j_{l}\right\}$;

4. for any $x, y$ in $i_{l}^{-1}\left(\Delta_{l, j}\right)$ and in a same unstable curve, there exists an unstable curve containing $x$ and $y$ and contained in $M \backslash R_{-l, 0}$;

5. if $\tilde{T}_{1}^{-1}\left(\Delta_{0}\right) \cap \Delta_{l, j} \neq \emptyset$, then there exists an integer $i \geq 0$ such that $\tilde{T}_{1}^{-1}\left(\Delta_{0}\right) \cap \Delta_{l, j}=\Lambda_{i} \times\left\{r_{i}-1\right\}$.

For any $X, Y \in \tilde{M}_{1}$, we define the separation time $s(X, Y)$ between $X$ and $Y$ as follows:

$$
s(X, Y):=\max \left\{n \geq 0: \tilde{T}_{1}^{n}(Y) \in \mathcal{D}\left(\tilde{T}_{1}^{n}(X)\right)\right\}
$$

The following fact shall be useful in our proof:

Fact A.4. Let $n \geq 0$ be an integer. Let $X$ and $Y$ be two points in $\tilde{M}_{1}$ such that $s(X, Y) \geq n$. Then, the intersection point $z$ of the curves $\gamma^{s}\left(\tilde{\pi}_{1}(X)\right)$ and $\gamma^{u}\left(\tilde{\pi}_{1}(Y)\right)$ exists. Moreover, $T^{n}(z)$ and $T^{n}\left(\tilde{\pi}_{1}(Y)\right)$ are both contained in a same unstable curve.

Let us write $d:=\operatorname{gcd}\left(r_{i}\right)$. 


\section{An extension of $(M, \nu, T)$}

We can show that the dynamical system $\left(\tilde{M}_{d}, \tilde{\nu}_{d}, \tilde{T}_{d}\right)$, defined as follows, is an extension of $\left(M, \nu, T^{d}\right)$ by $\tilde{\pi}_{d}:=\tilde{\pi}_{1} \mid \tilde{M}_{d}:$

- $\tilde{M}_{d}:=\bigcup_{l>0} \Delta_{l d}$

- $\tilde{\mu}_{d}:=\left(\tilde{\nu}_{1}\right)_{\mid \tilde{M}_{d}}$ and $\tilde{\nu}_{d}:=d . \tilde{\mu}_{d}$ is the probability measure proportional to $\tilde{\mu}_{d}$;

- $\tilde{T}_{d}:=\left(\tilde{T}_{1}^{d}\right)_{\mid \tilde{M}_{d}}$.

\section{A factor with a quasicompact transfer operator}

We consider the factor $\left(\hat{M}_{d}, \hat{\nu}_{d}, \hat{T}_{d}\right)$ of $\left(\tilde{M}_{d}, \tilde{\nu}_{d}, \tilde{T}_{d}\right)$ given by the canonical projection $\hat{\pi}_{d}: \tilde{M}_{d} \rightarrow \hat{M}_{d}$, where $\hat{M}_{d}$ is the set of the $\mathcal{R}$-classes of $\tilde{M}_{d}$, for the binary relation $\mathcal{R}$ defined on $\tilde{M}_{d}$ by:

$$
(x, l) \mathcal{R}\left(x^{\prime}, l^{\prime}\right) \Leftrightarrow l=l^{\prime} \text { and } x, x^{\prime} \text { are in a same } \gamma^{s} \in \Gamma^{s} .
$$

Young defines a natural measure $\hat{m}$ on $\hat{M}_{d}$ such that $\hat{\nu}_{d}$ is absolutely continuous relatively to $\hat{m}$ and such that the density $\hat{\rho}:=\frac{\mathrm{d} \hat{\nu}_{d}}{\mathrm{~d} \hat{m}}$ satisfies:

- $c_{0}{ }^{-1} \leq \hat{\rho} \leq c_{0}$, for some real number $c_{0}>1$;

- $|\hat{\rho}(\hat{x})-\hat{\rho}(\hat{y})| \leq c_{1} \alpha_{0}^{\hat{s}(\hat{x}, \hat{y})} \hat{\rho}(\hat{x})$, for some real numbers $c_{1}>0$ and $\left.\alpha_{0} \in\right] 0 ; 1[$;

with $\hat{s}\left(\hat{\pi}_{d}(x), \hat{\pi}_{d}(y)\right)=s(x, y)$. We shall write $\hat{\Delta}_{l . d}:=\hat{\pi}_{d}\left(\Delta_{l . d}\right)$ and $\hat{\Delta}_{l . d, j}:=\hat{\pi}_{d}\left(\Delta_{l . d, j}\right)$. Let us fix $\alpha_{1}:=$ $\max \left(\alpha, \alpha_{0}\right)$. For any real numbers $\left.\beta \in\right] 0 ; 1\left[\right.$ and $\varepsilon>0$, we define the functional space $\mathcal{V}_{(\beta, \varepsilon)}$ as follows:

$$
\mathcal{V}_{(\beta, \varepsilon)}:=\left\{\hat{f}: \hat{M}_{d} \rightarrow \mathbf{C} \text { measurable, }\|\hat{f}\|_{\mathcal{V}_{(\beta, \varepsilon)}}<+\infty\right\},
$$

where $\|\hat{f}\|_{\mathcal{V}_{(\beta, \varepsilon)}}:=\|\hat{f}\|_{(\beta, \varepsilon, \infty)}+\|\hat{f}\|_{(\beta, \varepsilon, h)}$, with

$$
\begin{aligned}
\|\hat{f}\|_{(\beta, \varepsilon, \infty)} & :=\sup _{l \geq 0} \| \hat{f}_{\mid \hat{\Delta}_{l . d} \|_{\infty}} \mathrm{e}^{-l . d . \varepsilon}, \\
\|\hat{f}\|_{(\beta, \varepsilon, h)} & :=\sup _{l \geq 0 ; j=1, \ldots, j_{l . d}} \sup _{\hat{x}, \hat{y} \in \hat{\Delta}_{l . d, j}} \frac{|\hat{f}(\hat{x})-\hat{f}(\hat{y})|}{\beta^{\left.d ! \frac{\hat{s}(\hat{x}, \hat{y})}{d}\right\rfloor}} \mathrm{e}^{-l . d . \varepsilon} .
\end{aligned}
$$

The transfer operator associated to $\hat{T}_{d}$ relatively to $\hat{m}$ shall be written $P$. Young shows that we can find two real numbers $\beta \in] \alpha_{1} ; 1\left[\right.$ and $\varepsilon_{0}>0$ such that, for any real number $\left.\varepsilon \in\right] 0 ; \varepsilon_{0}[$, the three following points hold:

- there exists a real $C_{0}>0$ satisfying $\|\cdot\|_{L^{1}(\hat{m})} \leq C_{0}\|\cdot\|_{\mathcal{V}_{(\beta, \varepsilon)}}$;

- there exist two real numbers $\left.\tau_{1} \in\right] 0 ; 1\left[\right.$ and $C_{1}>0$ such that, for any integer $n \geq 0$ and for any $\hat{f} \in \mathcal{V}_{(\beta, \varepsilon)}$ satisfying $\int_{\hat{M}_{d}} \hat{f} \mathrm{~d} \hat{m}=0$, we have

$$
\left\|P^{n} \hat{f}\right\|_{\mathcal{V}_{(\beta, \varepsilon)}} \leq C_{1} \tau_{1}^{n}\|\hat{f}\|_{\mathcal{V}_{(\beta, \varepsilon)}}
$$

- we have $P(\hat{f})(\hat{x})=\sum_{\hat{z}: \hat{T}_{d}(\hat{z})=\hat{x}} \xi(\hat{z}) \hat{f}(\hat{z})$, with $\left|\log \frac{\xi(\hat{x})}{\xi(\hat{y})}\right| \leq C_{2} \alpha_{1} \hat{s}(\hat{x}, \hat{y})-1$, for any $\hat{x}$ and $\hat{y}$ in a same $\hat{\Delta}_{l, j}$. 


\section{Appendix B. Proof of the Strong DeCorrelation Property For Billiard TRANSFORMATION}

\section{An exponential rate of decorrelation for $(M, \nu, T)$}

Theorem B.1. Let $\kappa \in] 0 ; \frac{1}{2}\left[\right.$ be a real number. There exists a constant $L_{\eta, \kappa}>0$ such that, for any integers $\tilde{m}_{1}, \tilde{m}_{2}$, any functions $\phi$ and $\psi$ in $\mathcal{H}_{\eta, \tilde{m}_{1} . d}$ and in $\mathcal{H}_{\eta, \tilde{m}_{2} . d}$ respectively and any integer $n \geq 0$, we have

$$
\left|\operatorname{Cov}_{\nu}\left(\phi, \psi \circ T^{n . d}\right)\right| \leq L_{\eta}\left(\|\phi\|_{\infty} C_{\psi}+C_{\phi}\|\psi\|_{\infty}+\|\phi\|_{\infty} \cdot\|\psi\|_{\infty}\right) \tau_{0}^{n-\frac{\tilde{m}_{1}}{1-2 \kappa}}
$$

with $\tau_{0}:=\max \left(\alpha^{\kappa \eta \cdot d}, \tau_{1}^{1-2 \kappa}\right)$.

Before establishing this result, we give the idea of its proof. We shall suppose $n(1-2 \kappa) \geq \tilde{m}_{1}$ and shall show how the study of $\operatorname{Cov}_{\nu}\left(\phi, \psi \circ T^{n . d}\right)$ leads us, after approximations, to the study of a quantity of the form $\mathbf{E}_{\hat{m}}\left[\hat{f} . \hat{g} \circ \hat{T}_{d}^{n}\right]$, where $\hat{f}$ and $\hat{g}$ are two bounded functions defined on $\hat{M}_{d}$ such that $P^{n_{1}} \hat{f}$ is $\hat{m}$-centered and is in $\mathcal{V}_{(\beta, \varepsilon)}$ with $n_{1}=2\lfloor\kappa n\rfloor+\tilde{m}_{1}$. Therefore, we shall get:

$$
\begin{aligned}
\left|\mathbf{E}_{\hat{m}}\left[\hat{f} . \hat{g} \circ \hat{T}_{d}^{n}\right]\right| & =\left|\mathbf{E}_{\hat{m}}\left[P^{n}(\hat{f}) \cdot \hat{g}\right]\right| \leq\|\hat{g}\|_{\infty}\left\|P^{n}(\hat{f})\right\|_{1} \\
& \leq\|\hat{g}\|_{\infty} C_{0}\left\|P^{n}(\hat{f})\right\|_{\mathcal{V}_{(\beta, \varepsilon)}} \leq\|\hat{g}\|_{\infty} C_{0} C_{1} \tau_{1}^{n-n_{1}}\left\|P^{n_{1}}(\hat{f})\right\|_{\mathcal{V}_{(\beta, \varepsilon)}}
\end{aligned}
$$

Proof. Let $\tilde{m}_{1}, \tilde{m}_{2}, \kappa, \phi$ and $\psi$ be as in the statement of the theorem. If $n(1-2 \kappa)<\tilde{m}_{1}$, then we have

$$
\left|\operatorname{Cov}_{\nu}\left(\phi, \psi \circ T^{n . d}\right)\right| \leq\|\phi\|_{\infty} \cdot\|\psi\|_{\infty} \leq\|\phi\|_{\infty} \cdot\|\psi\|_{\infty} \tau_{0}{ }^{-\frac{\tilde{m}_{1}}{1-2 \kappa}} \tau_{0}{ }^{n} .
$$

In the following, we shall suppose $n(1-2 \kappa) \geq \tilde{m}_{1}$. We denote $k=k_{n}:=\lfloor\kappa n\rfloor$. We have $n \geq 2 \kappa n+\tilde{m}_{1} \geq 2 k+\tilde{m}_{1}$. Therefore

$$
\left|\operatorname{Cov}_{\nu}\left(\phi, \psi \circ T^{n . d}\right)\right|=\left|\operatorname{Cov}_{\tilde{\nu}_{d}}\left(\tilde{\phi} \circ \tilde{T}_{d}^{k},\left(\tilde{\psi} \circ \tilde{T}_{d}^{k}\right) \circ \tilde{T}_{d}^{n}\right)\right|
$$

with $\tilde{\phi}:=\phi \circ \tilde{\pi}_{d}$ and $\tilde{\psi}:=\psi \circ \tilde{\pi}_{d}$. So, we have $\left|\operatorname{Cov}_{\nu}\left(\phi, \psi \circ T^{n . d}\right)\right| \leq A_{n}+B_{n}+C_{n}$, with $A_{n}, B_{n}$ and $C_{n}$ defined as follows:

1. we write $A_{n}:=\left|\operatorname{Cov}_{\tilde{\nu}_{d}}\left(\tilde{\phi} \circ \tilde{T}_{d}^{k},\left(\tilde{\psi} \circ \tilde{T}_{d}^{k}\right) \circ \tilde{T}_{d}^{n}\right)-\operatorname{Cov}_{\tilde{\nu}_{d}}\left(\tilde{\phi} \circ \tilde{T}_{d}^{k}, \hat{\psi}_{k} \circ \tilde{T}_{d}^{n}\right)\right|$ where $\hat{\psi}_{k}(x)$ is the infimum of $\tilde{\psi} \circ \tilde{T}_{d}^{k}=\psi \circ T^{k d} \circ \tilde{\pi}_{d}$ on the atom of $\mathcal{M}_{2 k+\tilde{m}_{2}}$ containing $x$, where we have written

$$
\mathcal{M}_{2 k+\tilde{m}_{2}}:=\left(\bigvee_{i=0}^{\left(2 k+\tilde{m}_{2}\right) d} \tilde{T}_{1}^{-i} \mathcal{D}\right)_{\mid \tilde{M}_{d}}
$$

We shall use the regularity of $\psi$ to get an upper-bound for $A_{n}$. We recall that, by hypothesis, $x \mapsto \psi(x)$ is Hölderian of order $\eta$ in $\left(x, T(x), \ldots, T^{\tilde{m}_{2} \cdot d}(x)\right)$. Moreover, we shall see that each atom of $T^{k d+j}\left(\tilde{\pi}_{d}\left(\mathcal{M}_{2 k+\tilde{m}_{2}}\right)\right)$ (for $j=0, \ldots, \tilde{m}_{2} d$ ) is contained in a connected component of $M \backslash R_{-s_{j}, 0}$ and has a diameter less than $2 C \alpha_{1}{ }^{k d}$, with $s_{j}:=\left(2 k+\tilde{m}_{2}\right) d-(k d+j) \geq k d$. Indeed, let $Y_{1}$ and $Y_{2}$ be two points in a same atom of $\mathcal{M}_{2 k+\tilde{m}_{2}}$. Then, we have $s\left(Y_{1}, Y_{2}\right) \geq 2 k+\tilde{m}_{2}$. Therefore, according to Fact A.4, the intersection point $y_{3}$ of $\gamma^{u}\left(\tilde{\pi}_{d}\left(Y_{1}\right)\right)$ with $\gamma^{s}\left(\tilde{\pi}_{d}\left(Y_{2}\right)\right)$ exists. Since $y_{3}$ and $\tilde{\pi}_{d}\left(Y_{2}\right)$ are both contained in a same stable curve and according to Proposition A.2, we have

$$
d\left(T^{k d+j}\left(y_{3}\right), T^{k d+j}\left(\tilde{\pi}_{d}\left(Y_{2}\right)\right)\right) \leq C \alpha^{k d+j} \leq C \alpha^{k d}
$$


Moreover, according to Fact A.4, $T^{\left(2 k+\tilde{m}_{2}\right) d}\left(y_{3}\right)$ and $T^{\left(2 k+\tilde{m}_{2}\right) d}\left(\tilde{\pi}_{1}\left(Y_{1}\right)\right)$ are both contained in a same unstable curve. So we have:

$$
d\left(T^{k d+j}\left(y_{3}\right), T^{k d+j}\left(\tilde{\pi}_{d}\left(Y_{1}\right)\right)\right) \leq C \alpha^{s_{j}} \leq C \alpha^{k d}
$$

As $\psi$ is in $\mathcal{H}_{\eta, \tilde{m}_{2} d}$, according to the foregoing, we have

$$
\left\|\tilde{\psi} \circ \tilde{T}_{d}^{k}-\hat{\psi}_{k}\right\|_{\infty} \leq C_{\psi}\left(2 C \alpha^{k d}\right)^{\eta} \leq K_{\eta} C_{\psi} \tau_{0}{ }^{n}
$$

with $K_{\eta}:=(2 C)^{\eta} \alpha^{-\eta d}$. We get

$$
A_{n} \leq\|\phi\|_{\infty}\left\|\tilde{\psi} \circ \tilde{T}_{d}^{k}-\hat{\psi}_{k}\right\|_{\infty} \leq K_{\eta}\|\phi\|_{\infty} C_{\psi} \tau_{0}^{n}
$$

2. We write $B_{n}:=\left|\operatorname{Cov}_{\tilde{\nu}_{d}}\left(\tilde{\phi} \circ \tilde{T}_{d}^{k}, \hat{\psi}_{k} \circ \tilde{T}_{d}^{n}\right)-\operatorname{Cov}_{\tilde{\nu}_{d}}\left(\hat{\phi}_{k}, \hat{\psi}_{k} \circ \tilde{T}_{d}^{n}\right)\right|$ where $\hat{\phi}_{k}(x)$ is the infimum of $\tilde{\phi} \circ \tilde{T}_{d}^{k}$ on the atom of $\mathcal{M}_{2 k+\tilde{m}_{1}}:=\left(\bigvee_{i=0}^{\left(2 k+\tilde{m}_{1}\right) d} \tilde{T}_{1}^{-i} \mathcal{D}\right)_{\mid \tilde{M}_{d}}$ containing $x$. As previously, we can show that we have $B_{n} \leq K_{\eta} C_{\phi}\|\psi\|_{\infty} \tau_{0}^{n}$

3. We shall now give an upper bound for the following quantity:

$$
\begin{aligned}
C_{n} & :=\left|\operatorname{Cov}_{\tilde{\nu}_{d}}\left(\hat{\phi}_{k}, \hat{\psi}_{k} \circ \tilde{T}_{d}^{n}\right)\right| \\
& =\left|\int_{\tilde{M}_{d}} \hat{\psi}_{k} \circ \tilde{T}_{d}^{n} \cdot \hat{\phi}_{k} \mathrm{~d} \tilde{\nu}_{d}-\int_{\tilde{M}_{d}} \hat{\phi}_{k} \mathrm{~d} \tilde{\nu}_{d} \cdot \int_{\tilde{M}_{d}} \hat{\psi}_{k} \mathrm{~d} \tilde{\nu}_{d}\right| \\
& =\left|\int_{\hat{M}_{d}} \hat{\psi}_{k} \circ \hat{T}_{d}^{n} \cdot \hat{\phi}_{k} \mathrm{~d} \hat{\nu}_{d}-\int_{\hat{M}_{d}} \hat{\phi}_{k} \mathrm{~d} \hat{\nu}_{d} \cdot \int_{\hat{M}_{d}} \hat{\psi}_{k} \mathrm{~d} \hat{\nu}_{d}\right| \\
& \leq\left|\int_{\hat{M}_{d}} \hat{\psi}_{k} \cdot P^{n}\left(\hat{\phi}_{k} \hat{\rho}\right) \mathrm{d} \hat{m}-\int_{\hat{M}_{d}} \hat{\phi}_{k} \mathrm{~d} \hat{\nu}_{d} \cdot \int_{\hat{M}_{d}} \hat{\psi}_{k} \mathrm{~d} \hat{\nu}_{d}\right| \\
& \leq\left|\int_{\hat{M}_{d}} \hat{\psi}_{k} \cdot\left(P^{n}\left(\hat{\phi}_{k} \hat{\rho}\right)-\left(\int_{\hat{M}_{d}} \hat{\phi}_{k} \hat{\rho} \mathrm{d} \hat{m}\right) \hat{\rho}\right) \mathrm{d} \hat{m}^{-1}\right| \\
& \left.\left.\leq\|\psi\|_{\infty} C_{0} \| P^{n}\left(\hat{\phi}_{k} \hat{\rho}\right)-\left(\int_{\hat{M}_{d}} \hat{\phi}_{k} \hat{\rho} \mathrm{d} \hat{m}\right) \hat{\rho}_{\mathcal{\nu}_{(\beta, \varepsilon)}} \hat{\phi}_{k} \text { the map } \hat{\phi}_{k} \circ \hat{\pi}_{1}^{-1}, \hat{\phi}_{k} \hat{\rho} \mathrm{d} \hat{m}\right) \hat{\rho}\right) \|_{\mathcal{V}_{(\beta, \varepsilon)}}
\end{aligned}
$$

since $\left(\hat{\phi}_{k}-\int_{\hat{M}_{d}} \hat{\phi}_{k} \hat{\rho} \mathrm{d} \hat{m}\right) \hat{\rho}$ is $\hat{m}$-centered and we shall see that $P^{2 k+\tilde{m}_{1}}\left(\hat{\phi}_{k} \hat{\rho}\right)$ is in $\mathcal{V}_{(\beta, \varepsilon)}$. Let $l \geq 0$ and $j=1, \ldots, j_{l d}$ be two integers. We denote by $\mathcal{A}_{l d, j}$ the set of atoms $A$ of $\hat{\mathcal{M}}_{2 k+\tilde{m}_{1}}:=\hat{\pi}_{d}\left(\mathcal{M}_{2 k+\tilde{m}_{1}}\right)$ such that $\hat{T}_{d}^{2 k+\tilde{m}_{1}}(A) \subseteq \hat{\Delta}_{l . d, j}$. Let $A$ be an atom of $\mathcal{A}_{l d, j}$. Then, the map $\hat{T}_{d}^{2 k+\tilde{m}_{1}}$ defines a one-to-one map from $A$ onto $\hat{\Delta}_{l d, j}$. Indeed, point 5 of Properties A.3, the fact that each $\Lambda_{i}$ is a $s$-sub-rectangle and that $T^{r_{i}}\left(\Lambda_{i}\right)$ is a $u$-sub-rectangle insure us that $\hat{T}_{d}$ defines a one-to-one map from each $\hat{B}\left(\right.$ in $\hat{\pi}_{d}\left(\bigvee_{j "}^{d}=0 \tilde{T}_{1}^{-j}{ }^{\prime \prime}(\mathcal{D})\right)$ and such that $\left.\hat{T}_{d}(\hat{B}) \subseteq \hat{\Delta}_{0}\right)$ onto $\hat{\Delta}_{0}$. We denote by $\hat{T}_{(A, d)}^{-\left(2 k+\tilde{m}_{1}\right)}$ the inverse map of $\left(\hat{T}_{d}^{2 k+\tilde{m}_{1}}\right)_{\mid A}$. We notice 
that, for any $\hat{x} \in \hat{\Delta}_{l d, j}$, we have

$$
P^{2 k+\tilde{m}_{1}}(\hat{f})(\hat{x})=\sum_{A \in \mathcal{A}_{l d, j}} \xi_{A}(\hat{x}) \hat{f}\left(\hat{T}_{(A, d)}^{-\left(2 k+\tilde{m}_{1}\right)}(\hat{x})\right),
$$

where $\xi_{A}(\hat{x}):=\prod_{i=0}^{2 k+\tilde{m}_{1}-1} \xi\left(\hat{T}_{d}^{i}\left(\hat{T}_{(A, d)}^{-\left(2 k+\tilde{m}_{1}\right)}(\hat{x})\right)\right)$. Since we have $P(\hat{\rho})=\hat{\rho}, \xi_{A} \geq 0$ and $\hat{\rho} \geq 0$, we have

$$
\begin{aligned}
\left\|P^{2 k+\tilde{m}_{1}}\left(\hat{\phi}_{k} \hat{\rho}\right)\right\|_{(\beta, \varepsilon, \infty)} & =\sup _{l, j}\left|P^{2 k+\tilde{m}_{1}}\left(\hat{\phi}_{k} \hat{\rho}\right)_{\mid \hat{\Delta}_{l, d, j}}\right|_{\infty} \mathrm{e}^{-l . d \varepsilon} \\
& \leq \sup _{l, j}\|\phi\|_{\infty}\left|P^{2 k+\tilde{m}_{1}}(\hat{\rho})_{\mid \hat{\Delta}_{l . d, j}}\right|_{\infty} \mathrm{e}^{-l . d \varepsilon} \leq c_{0}\|\phi\|_{\infty} .
\end{aligned}
$$

According to the foregoing, for any $\hat{x}, \hat{y} \in \hat{\Delta}_{l, j}$, we have

$$
\left|\log \left(\frac{\xi_{A}(\hat{x})}{\xi_{A}(\hat{y})}\right)\right| \leq \frac{C_{2} \alpha_{1}{ }^{\hat{s}(\hat{x}, \hat{y})}}{1-\alpha_{1}}
$$

and so $\left|\xi_{A}(\hat{y})-\xi_{A}(\hat{x})\right| \leq C_{1}^{\prime} \xi_{A}(\hat{x}) \alpha_{1}{ }^{\hat{s}(\hat{x}, \hat{y})}$, for some constant $C_{1}^{\prime}>0$ independent of $n$ and of $A$. We denote by $c_{A, l . d, j}$ the constant to which $\hat{\phi}_{k}$ is equal on $A$ and $\hat{\rho}_{2 k+\tilde{m}_{1}, l . d, j}^{(A)}:=\hat{\rho} \circ \hat{T}_{(A, d)}^{-\left(2 k+\tilde{m}_{1}\right)}$. We get

$$
\begin{aligned}
& \left\|P^{2 k+\tilde{m}_{1}}\left(\hat{\phi}_{k} \hat{\rho}\right)\right\|_{(\beta, \varepsilon, h)}=\sup _{l, j}\left(\sup _{\hat{x}, \hat{y} \in \hat{\Delta}_{l d, j}} \frac{\left|P^{2 k+\tilde{m}_{1}}\left(\hat{\phi}_{k} \hat{\rho}\right)(\hat{x})-P^{2 k+\tilde{m}_{1}}\left(\hat{\phi}_{k} \hat{\rho}\right)(\hat{y})\right|}{\beta^{\left.d ! \frac{\hat{s}(\hat{x}, \hat{y})}{d}\right\rfloor}}\right) \mathrm{e}^{-l . d \varepsilon} \\
& \leq \sup _{l, j} \sup _{\hat{x}, \hat{y} \in \hat{\Delta}_{l . d, j}} \sum_{A \in \mathcal{A}_{l . d, j}}\left|c_{A, j, l . d}\right| \cdot \frac{\left|\xi_{A}(\hat{x}) \hat{\rho}_{2 k+\tilde{m}_{1}, l . d, j}^{(A)}(\hat{x})-\xi_{A}(\hat{y}) \hat{\rho}_{2 k+\tilde{m}_{1}, l . d, j}^{(A)}(\hat{y})\right|}{\beta^{d\left\lfloor\frac{\hat{s}(\hat{x}, \hat{y})}{d}\right\rfloor}} \mathrm{e}^{-l . d \varepsilon} \\
& \leq \sup _{l, j} \sup _{\hat{x}, \hat{y} \in \hat{\Delta}_{l . d, j}}\|\phi\|_{\infty} \sum_{A \in \mathcal{A}_{l . d, j}} \frac{\left|\xi_{A}(\hat{x}) \hat{\rho}_{2 k++\tilde{m}_{1}, l . d, j}^{(A)}(\hat{x})-\xi_{A}(\hat{y}) \hat{\rho}_{2 k+\tilde{m}_{1}, l . d, j}^{(A)}(\hat{y})\right|}{\left.\beta^{d ! \frac{\hat{s}(\hat{x}, \hat{y})}{d}}\right\rfloor} \mathrm{e}^{-l . d \varepsilon} \\
& \leq\left(C_{1}^{\prime}+c_{1}+c_{1} C_{1}^{\prime}\right) \sup _{l, j} \sup _{\hat{x}, \hat{y} \in \hat{\Delta}_{l d, j}}\|\phi\|_{\infty} \sum_{A \in \mathcal{A}_{l d, j}} \frac{\alpha_{1}^{\hat{s}(\hat{x}, \hat{y})} \xi_{A}(\hat{x}) \hat{\rho}_{2 k+\tilde{m}_{1}, l d, j}^{(A)}(\hat{x})}{\beta^{d\left\lfloor\frac{\hat{s}(\hat{x}, \hat{y})}{d}\right\rfloor}} \mathrm{e}^{-l d \varepsilon} \\
& \leq\left(C_{1}^{\prime}+c_{1}+c_{1} C_{1}^{\prime}\right) \sup _{l, j} \sup _{\hat{x}, \hat{y} \in \hat{\Delta}_{l . d, j}}\|\phi\|_{\infty} \frac{\alpha_{1}^{\hat{s}(\hat{x}, \hat{y})} \hat{\rho}(\hat{x})}{\beta^{d\left\lfloor\frac{\hat{s}(\hat{x}, \hat{y}}{d}\right\rfloor}} \mathrm{e}^{-l . d \varepsilon} \\
& \leq\left(C_{1}^{\prime}+c_{1}+c_{1} C_{1}^{\prime}\right) \sup _{l, j} \sup _{\hat{x}, \hat{y} \in \hat{\Delta}_{l . d, j}}\|\phi\|_{\infty} \frac{\alpha_{1}^{\hat{s}(\hat{x}, \hat{y})}}{\alpha_{1} \hat{s}(\hat{x}, \hat{y})} \hat{\rho}(\hat{x}) \mathrm{e}^{-l . d \varepsilon} \\
& \leq\left(C_{1}^{\prime}+c_{1}+c_{1} C_{1}^{\prime}\right)\|\phi\|_{\infty} c_{0} .
\end{aligned}
$$

In particular, we have shown that $P^{2 k+\tilde{m}_{1}}\left(\hat{\phi}_{k} \hat{\rho}\right)$ is in $\mathcal{V}_{(\beta, \varepsilon)}$.

According to the foregoing, $\left|\operatorname{Cov}_{\nu}\left(\phi, \psi \circ T^{n d}\right)\right|$ is less than:

$K_{\eta}\left(\|\phi\|_{\infty} C_{\psi}+C_{\phi}\|\psi\|_{\infty}\right) \tau_{0}^{n}+C_{0} C_{1}\left(C_{1}^{\prime}+c_{1}+c_{1} C_{1}^{\prime}\right) c_{0} \tau_{1}^{(1-2 \kappa) n-\tilde{m}_{1}}\|\phi\|_{\infty}\|\psi\|_{\infty}$

$$
\leq K_{\eta}\left(\|\phi\|_{\infty} C_{\psi}+C_{\phi}\|\psi\|_{\infty}\right) \tau_{0}{ }^{n}+C_{0} C_{1}\left(C_{1}^{\prime}+c_{1}+c_{1} C_{1}^{\prime}\right) c_{0} \tau_{0}{ }^{n-\frac{\tilde{m}_{1}}{1-2 \kappa}}\|\phi\|_{\infty}\|\psi\|_{\infty} .
$$




\section{End of the proof of proposition 4.2.1}

Proposition 4.2.1 is an easy consequence of the following result (with $r=\frac{1}{1-2 \kappa}$ ).

Corollary B.2. Let $m_{1}, m_{2} \geq 0$ be two integers and $\left.\kappa \in\right] 0 ; 1 / 2[$ be a real number. For any functions $\phi$ and $\psi$ in $\mathcal{H}_{\eta, m_{1}}$ and in $\mathcal{H}_{\eta, m_{2}}$ respectively and any integer $n \geq 0$, we have

$$
\left|\operatorname{Cov}_{\nu}\left(\phi, \psi \circ T^{n}\right)\right| \leq C_{\eta, \kappa}\left(\|\phi\|_{\infty} C_{\psi}+C_{\phi}\|\psi\|_{\infty}+\|\phi\|_{\infty} \cdot\|\psi\|_{\infty}\right) \tau_{0}^{\prime n-\frac{m_{1}}{1-2 \kappa}}
$$

with $\tau_{0}^{\prime}:=\max \left(\alpha_{1}^{-\kappa \eta}, \tau_{1}^{\frac{1-2 \kappa}{d}}\right)=\tau_{0}^{\frac{1}{d}}$ et $C_{\eta, \kappa}:=L_{\eta, \kappa} \tau_{0}^{\prime-\frac{d}{1-2 \kappa}-d+1}$.

Proof. For any integer $l=0, \ldots, d-1$, we apply the foregoing to the couple $\left(\phi, \psi \circ T^{l}\right)$. Indeed, $\phi$ is in $\mathcal{H}_{\eta, m_{1}}$ so in $\mathcal{H}_{\eta,\left\lceil\frac{m_{1}}{d}\right\rceil d}$ with $C_{\phi}^{\left(\eta,\left\lceil\frac{m_{1}}{d}\right\rceil d\right)} \leq C_{\phi}^{\left(\eta, m_{1}\right)}$. On the other hand, $\psi \circ T^{l}$ is in $\mathcal{H}_{\eta,\left\lceil\frac{m_{2}+l}{d}\right\rceil d}$, with $C_{\psi \circ T^{l}}^{\left(\eta,\left\lceil\frac{m_{2}+l}{d}\right\rceil d\right)} \leq C_{\psi}^{\left(\eta, m_{2}\right)}$. Consequently, for any integer $k \geq 0$, we have:

$$
\begin{aligned}
\left|\operatorname{Cov}_{\nu}\left(\phi, \psi \circ T^{k d+l}\right)\right| & =\left|\operatorname{Cov}_{\nu}\left(\phi,\left(\psi \circ T^{l}\right) \circ T^{k d}\right)\right| \\
& \leq L_{\eta, \kappa}\left(\|\phi\|_{\infty} C_{\psi}+C_{\phi}\|\psi\|_{\infty}+\|\phi\|_{\infty}\|\psi\|_{\infty}\right) \tau_{0}{ }^{k} \tau_{0}{ }^{-\frac{\left\lceil\frac{m_{1}}{d}\right\rceil}{1-2 \kappa}} \\
& \leq L_{\eta, \kappa}\left(\|\phi\|_{\infty} C_{\psi}+C_{\phi}\|\psi\|_{\infty}+\|\phi\|_{\infty}\|\psi\|_{\infty}\right) \tau_{0}^{\prime k d} \tau_{0}^{\prime-\frac{\left\lceil\frac{m_{1}}{d}\right] d}{1-2 \kappa}} \\
& \leq L_{\eta, \kappa}\left(\|\phi\|_{\infty} C_{\psi}+C_{\phi}\|\psi\|_{\infty}+\|\phi\|_{\infty}\|\psi\|_{\infty}\right) \tau_{0}^{\prime k d} \tau_{0}^{\prime-\frac{m_{1}+d}{1-2 \kappa}} \\
& \leq L_{\eta, \kappa}\left(\|\phi\|_{\infty} C_{\psi}+C_{\phi}\|\psi\|_{\infty}+\|\phi\|_{\infty}\|\psi\|_{\infty}\right) \tau_{0}^{\prime k d+l} \tau_{0}^{\prime-\frac{m_{1}+d}{1-2 \kappa}} \tau_{0}^{\prime-d+1} .
\end{aligned}
$$

\section{Appendix C. Sketch of the proof of the martingale Approximations}

In this section, we only give the ideas of adaptations to do to [18] in order to prove Theorem 5.3.6 and the following result (details of are done in Chap. 4 of [14]).

Theorem C.1. Let a $\nu$-centered function $f: M \rightarrow \mathbf{R}$ in $\mathcal{H}_{\eta, m_{0}}$ be given.

There exist an invertible extension $(\dot{M}, \dot{\nu}, \dot{T})$ of $(M, \nu, T)$ given by $\dot{\pi}: \dot{M} \rightarrow M$ and two real valued functions $\dot{G}_{1}, \dot{H}_{1}$ belonging to all $L^{p}(\dot{M}, \dot{\nu})$ (with $p$ in $[1 ;+\infty[)$ and such that the following equality holds $\dot{\nu}$-almost surely:

$$
f \circ \dot{\pi}=\dot{G}_{1}+\dot{H}_{1}-\dot{H}_{1} \circ \dot{T}
$$

the function $\dot{G}_{1}$ generating a sequence of reversed martingale differences in $(\dot{M}, \dot{\nu}, \dot{T})$, i.e. satisfying $\mathbf{E}_{\dot{\nu}}\left[\dot{G}_{1} \mid \dot{G}_{1} \circ \dot{T}^{k}, k \geq 1\right]=0$.

There exists an invertible extension $\left(\dot{M}_{2}, \dot{\nu}_{2}, \dot{T}_{2}\right)$ of $(M, \nu, T)$ given by $\dot{\pi}_{2}: \dot{M}_{2} \rightarrow M$ and two real valued functions $\dot{G}_{2}, \dot{H}_{2}$ belonging to all $L^{p}\left(\dot{M}_{2}, \dot{\nu}_{2}\right)$ (with $p$ in $\left[1 ;+\infty\left[\right.\right.$ ) such that the following equality holds $\dot{\nu}_{2}$-almost surely:

$$
f \circ \dot{\pi}_{2}=\dot{G}_{2}+\dot{H}_{2}-\dot{H}_{2} \circ \dot{T}_{2},
$$

and such that the function $\dot{G}_{2}$ generates a sequence of martingale differences in $\left(\dot{M}_{2}, \dot{\nu}_{2}, \dot{T}_{2}\right)$, i.e. satisfies $\mathbf{E}_{\dot{\nu}_{2}}\left[\dot{G}_{2} \mid \dot{G}_{2} \circ\left(\dot{T}_{2}\right)^{-k}, k \geq 1\right]=0$. 
We notice that the fact that function $\dot{G}_{2}$ generates a sequence of martingale differences means that $\left(\sum_{k=l}^{l+n-1} \dot{G}_{2} \circ\left(\dot{T}_{2}\right)^{k}\right)_{n \geq 1}$ is a $\dot{\nu}_{2}$-centered martingale (for any integer $l$ ). In the same way, the fact that $\dot{G}_{1}$ generates a sequence of reversed martingale differences means that $\left(\sum_{k=l}^{l+n-1} \dot{G}_{1} \circ \dot{T}^{-k}\right)_{n \geq 1}$ is a $\dot{\nu}$-centered martingale (for any integer $l$ ).

\section{Reversed martingale}

We consider the factor $\left(\hat{M}_{1}, \hat{\nu}_{1}, \hat{T}_{1}\right)$ of $\left(\tilde{M}_{1}, \tilde{\nu}_{1}, \tilde{T}_{1}\right)$ given by the canonical projection $\hat{\pi}_{1}: \tilde{M}_{1} \rightarrow \hat{M}_{1}$, where $\hat{M}_{1}$ is the set of the $\mathcal{R}_{1}$-classes of $\tilde{M}_{1}$, for the binary relation $\mathcal{R}_{1}$ defined on $\tilde{M}_{1}$ by:

$$
(x, l) \mathcal{R}_{1}\left(x^{\prime}, l^{\prime}\right) \Leftrightarrow l=l^{\prime} \text { and } x, x^{\prime} \text { are in a same } \gamma^{s} \in \Gamma^{s} .
$$

Let $(\dot{M}, \dot{\nu}, \dot{T})$ be the natural extension of $\left(\tilde{M}_{1}, \tilde{\nu}_{1}, \tilde{T}_{1}\right)$ and $\dot{\pi}: \dot{M} \rightarrow \tilde{M}_{1}$ be the canonical projection. Let $f: M \rightarrow \mathbf{R}$ be a $\nu$-centered function in $\mathcal{H}_{\eta, m_{0}}$ (with a real number $\left.\left.\eta \in\right] 0 ; 1\right]$ and an integer $m_{0} \geq 0$ ). The dynamical system $(\dot{M}, \dot{\nu}, \dot{T})$ is an extension of $(M, \nu, T)$ by $\dot{\pi}_{1}:=\tilde{\pi}_{1} \circ \dot{\pi}$. We denote $\dot{f}:=f \circ \dot{\pi}_{1}$. Let $\hat{\mathcal{B}}$ be the Borel $\sigma$-algebra of $\hat{M}_{1}$ and $\dot{\mathcal{B}}$ its inverse image by $\hat{\pi}_{1} \circ \dot{\pi}$. In [18], Young proves that, if $f$ is Hölder continuous, we have:

$$
\sum_{j \geq 0}\left(\left\|\mathbf{E}_{\nu}\left[\dot{f} \mid \dot{T}^{j}(\dot{\mathcal{B}})\right]-\dot{f}\right\|_{L^{2}(\dot{M}, \dot{\nu})}+\left\|\mathbf{E}_{\dot{\nu}}\left[\dot{f} \mid \dot{T}^{-j}(\dot{\mathcal{B}})\right]\right\|_{L^{2}(\dot{M}, \dot{\nu})}\right)<+\infty
$$

From this and [8], she concludes that $\dot{f}$ is homologous, in $L^{2}(\dot{M}, \dot{\nu}, \dot{T})$ to a function generating a sequence of reversed martingale differences, i.e. there exists $g$ and $h$ in $L^{2}(\dot{M}, \dot{\nu})$ satisfying the following equation:

$$
\dot{f}=g+h-h \circ \dot{T}, \quad \dot{\nu}-a . e .
$$

and such that $\mathbf{E}_{\dot{\nu}}\left[g \mid g \circ \dot{T}^{k}, k \geq 1\right]=0$. We recall quickly how she establishes (1) and explain briefly how her proof can be adapted to our purpose. Let $p \geq 2$ be a real number.

1. First, using the contraction property of stable curves, she proves that, if $f$ is Hölder continuous of order $\eta$, then for any integer $j \geq 0$, we have:

$$
\left\|\mathbf{E}_{\dot{\nu}}\left[\dot{f} \mid \dot{T}^{j}(\dot{\mathcal{B}})\right]-\dot{f}\right\|_{L^{\infty}(\dot{M}, \dot{\nu})} \leq C_{f}^{(\eta)} C \alpha^{j}
$$

where $C_{f}^{(\eta)}$ is the Hölder coefficient of order $\eta$ of $f$. It is clear that (3) is still true with $C_{f}^{\left(\eta, m_{0}\right)}$ instead of $C_{f}^{(\eta)}$ if $f$ is in $\mathcal{H}_{\eta, m_{0}}$.

2. On the other hand, she notices that, for any integer $j \geq 0$, function $\mathbf{E}_{\dot{\nu}}\left[\dot{f} \mid \dot{T}^{-j}(\dot{\mathcal{B}})\right]$ is $\dot{\mathcal{B}}$-measurable and, using the fact that we have $\|\cdot\|_{L^{2}\left(\hat{\nu}_{d}\right)} \leq C_{0}\|\cdot\|_{\mathcal{V}_{(\beta, \varepsilon)}}$ (for any real number $\left.\left.\varepsilon \in\right] 0 ; \varepsilon_{0}\right]$ ), she gets, after calculations,

$$
\sum_{j \geq 0}\left\|\mathbf{E}_{\dot{\nu}}\left[\dot{f} \mid \dot{T}^{j}(\dot{\mathcal{B}})\right]\right\|_{L^{2}(\dot{M}, \dot{\nu})}<+\infty
$$

Her calculations can be done for $f$ in $\mathcal{H}_{\eta, m_{0}}$. Moreover, by choosing $\varepsilon>0$ small enough such that we have $\|\cdot\|_{L^{p}\left(\hat{M}_{d}, \hat{\nu}_{d}\right)} \leq D_{p}\|\cdot\|_{\mathcal{V}_{(\beta, \varepsilon)}}$ for some constant $D_{p}>0$, we deduce from her proof that there exist constants 
$D_{\eta, m_{0}, p}>0$ and $\left.\theta_{\eta, m_{0}, p} \in\right] 0 ; 1\left[\right.$ (independent of the real-valued and $\nu$-centered function $\left.f \in \mathcal{H}_{\eta, m_{0}}\right)$ such that, for any integer $j \geq 0$, we have:

$$
\left\|\mathbf{E}_{\dot{\nu}}\left[\dot{f} \mid \dot{T}^{j}(\dot{\mathcal{B}})\right]\right\|_{L^{p}(\dot{M}, \dot{\nu})} \leq D_{\eta, m_{0}, p}\left(\|f\|_{\infty}+C_{f}^{\left(\eta, m_{0}\right)}\right)\left(\theta_{\eta, m_{0}, p}\right)^{j} .
$$

3. We conclude that we have:

$$
\sum_{j \geq 0}\left(\left\|\mathbf{E}_{\dot{\nu}}\left[\dot{f} \mid \dot{T}^{j}(\dot{\mathcal{B}})\right]-\dot{f}\right\|_{L^{p}(\dot{M}, \dot{\nu})}+\left\|\mathbf{E}_{\dot{\nu}}\left[\dot{f} \mid \dot{T}^{-j}(\dot{\mathcal{B}})\right]\right\|_{L^{p}(\dot{M}, \dot{\nu})}\right)<+\infty
$$

According to Gordin's proof in [8], we conclude that functions $g$ and $h$ of $(2)$ are in $L^{p}(\dot{M}, \dot{\nu})$.

\section{Direct martingale}

We define the temporal symmetry $\mathcal{S}: M \rightarrow M$ by $\mathcal{S}(q, \vec{v})=\left(q, \vec{v}^{\prime}\right)$ with $\vec{v}^{\prime}:=2\langle\vec{n}(q), \vec{v}\rangle \vec{n}(q)-\vec{v}(c f$. picture).

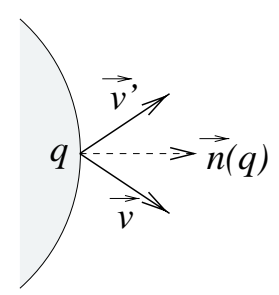

We notice that we have $\mathcal{S}^{-1}=\mathcal{S}$ and $T \circ \mathcal{S}=\mathcal{S} \circ T^{-1}$ (time reversing property). So, the dynamical system $\left(\dot{M}_{2}, \dot{\nu}_{2}, \dot{T}_{2}\right):=\left(\dot{M}, \dot{\nu}, \dot{T}^{-1}\right)$ is an extension of $(M, \nu, T)$ by $\dot{\pi}_{2}:=\mathcal{S} \circ \dot{\pi}_{1}$. Moreover we notice that, for any function $f \in \mathcal{H}_{\eta, m_{0}}$, function $\phi \circ \mathcal{S} \circ T^{m_{0}}$ is in $\mathcal{H}_{\eta, m_{0}}$ and that we have

$$
\phi \circ \dot{\pi}_{2}=\phi \circ \mathcal{S} \circ \dot{\pi}_{1}=\phi \circ \mathcal{S} \circ T^{m_{0}} \circ T^{-m_{0}} \circ \dot{\pi}_{1}=\left(\phi \circ \mathcal{S} \circ T^{m_{0}}\right) \circ \dot{\pi}_{1} \circ \dot{T}^{-m_{0}} .
$$

Thus, according to the foregoing, we conclude that there exists $G$ and $H$ belonging to all spaces $L^{p}(\dot{M}, \dot{\nu})$ (with $p \geq 1$ be any real number) and satisfying:

$$
\phi \circ \dot{\pi}_{2}=G+H-H \circ \dot{T}, \quad \dot{\nu}-a . e .
$$

and such that $\mathbf{E}_{\dot{\nu}}\left[G \mid G \circ \dot{T}^{k}, k \geq 1\right]=0$, i.e. $\mathbf{E}_{\dot{\nu}_{2}}\left[G \mid G \circ\left(\dot{T}_{2}\right)^{-k}, k \geq 1\right]=0$. Moreover, we have $\phi \circ \dot{\pi}_{2}=$ $G+H_{1}-H_{1} \circ \dot{T}_{2}, \dot{\nu}_{2}$-a.e., with $H_{1}:=-H \circ \dot{T}$.

The author expresses her gratitude to J.-P. Conze for his help and advices.

\section{REFERENCES}

[1] N. Bary, A treatise on trigonometric series, Vol. 1. Pergamon Press (1984).

[2] P. Billingsley, Convergence of probability measures. J. Wiley and Sons (1968).

[3] S. Le Borgne, Dynamique symbolique et propriétés stochastiques des automorphismes du tore : cas hyperbolique et quasihyperbolique, Ph.D. Thesis. University of Rennes I, France (1997).

[4] S. Le Borgne, Un problème de régularité dans l'équation de cobord, in Sémimaires de probabilités de Rennes. Université de Rennes 1 (1998); http://www.maths.univ-rennes1.fr/csp/1998/index.html 
[5] L.A. Bunimovich, N.I. Chernov and Y.G. Sinai, Statistical properties of two-dimensional hyperbolic billiards. Russian Math. Surveys 46 (1991) 47-106.

[6] L.A. Bunimovich and Y.G. Sinai, Statistical properties of Lorentz gaz with periodic configuration of scatterers. Comm. Math. Phys. 78 (1981) 479-497.

[7] N.I. Chernov and Y.G. Sinai, Ergodic properties of certain systems of two-dimensional discs and three-dimensional balls. Russian Math. Surveys 42 (1987) 181-207.

[8] M.I. Gordin, The Central Limit theorem for stationary processes. Soviet Math. Dokl. 10 (1969) 1174-1176.

[9] Y. Katznelson, Ergodic automorphisms of $\mathbf{T}^{n}$ are Bernoulli shifts. Israel J. Math. 10 (1971) 186-195.

[10] R.Z. Khas'minskii, On stochastic processes defined by differential equations with a small parameter (translation). Theory Probab. Appl. 11 (1966) 211-228.

[11] Y. Kifer, Limit theorem in averaging for dynamical systems. Ergodic Theory Dynam. Systems 15 (1995) 1143-1172.

[12] D.A. Lind, Dynamical properties of quasi hyperbolic toral automorphisms. Ergodic Theory Dynam. Systems 2 (1982) 49-68.

[13] V.P. Leonov, Quelques applications de la méthode des cumulants à la théorie des processus stochastiques stationnaires (in Russian). Nauka, Moscow (1964).

[14] F. Pène, Applications des propriétés stochastiques des systèmes dynamiques de type hyperbolique : ergodicité du billard dispersif dans le plan, moyennisation d'équations différentielles perturbées par un flot ergodique, Ph.D. Thesis. University of Rennes I, France (2000).

[15] F. Pène, Rates of convergence in the CLT for two-dimensional dispersive billiards. Comm. Math. Phys. 225 (2002) 91-119.

[16] D. Revuz and M. Yor, Continuous martingales and brownian motion. Springer-Verlag (1994).

[17] Y.G. Sinai, Dynamical systems with elastic reflections. Russian Math. Surveys 25 (1970) 137-189.

[18] L.-S. Young, Statistical properties of dynamical systems with some hyperbolicity. Ann. of Math. 147 (1998) 585-650. 